\title{
Systemic co-jumps
}

DOI:

10.1016/j.jfineco.2017.06.016

\section{Document Version}

Accepted author manuscript

Link to publication record in Manchester Research Explorer

\section{Citation for published version (APA):}

Caporin, M., Kolokolov, A., \& Renò, R. (2017). Systemic co-jumps. Journal of Financial Economics, 126(3), 563591. https://doi.org/10.1016/j.jfineco.2017.06.016

\section{Published in:}

Journal of Financial Economics

\section{Citing this paper}

Please note that where the full-text provided on Manchester Research Explorer is the Author Accepted Manuscript or Proof version this may differ from the final Published version. If citing, it is advised that you check and use the publisher's definitive version.

\section{General rights}

Copyright and moral rights for the publications made accessible in the Research Explorer are retained by the authors and/or other copyright owners and it is a condition of accessing publications that users recognise and abide by the legal requirements associated with these rights.

\section{Takedown policy}

If you believe that this document breaches copyright please refer to the University of Manchester's Takedown Procedures [http://man.ac.uk/04Y6Bo] or contact uml.scholarlycommunications@manchester.ac.uk providing relevant details, so we can investigate your claim.

\section{OPEN ACCESS}




\title{
Systemic co-jumps ${ }^{\text {th }}$
}

\author{
Massimiliano Caporin ${ }^{\mathrm{a}}$, Aleksey Kolokolov ${ }^{\mathrm{b}}$, Roberto Renò \\ ${ }^{a}$ University of Padova, Department of Statistical Sciences \\ ${ }^{b}$ Goethe University, SAFE Center \\ ${ }^{c}$ University of Verona, Department of Economics
}

\begin{abstract}
The simultaneous occurrence of jumps in several stocks can be associated with major financial news, triggers short-term predictability in stock returns, is correlated with sudden spikes of the variance risk premium, and determines a persistent increase (decrease) of stock variances and correlations when they come along with bad (good) news. These systemic events and their implications can be easily overlooked by traditional univariate jump statistics applied to stock indices. They are instead revealed in a clearly cut way by using a novel test procedure applied to individual assets, which is particularly effective on high-volume stocks.
\end{abstract}

Keywords: Jumps, Return predictability, Systemic events, Variance risk premium JEL: C58, G11, C14

\footnotetext{
"A previous version of this paper circulated with the title "Multi-jumps". We thank Federico Bandi, Kim Christensen, Fulvio Corsi, Tolga Ergun, Giampiero Gallo, Giulio Girardi, Cecilia Mancini, Giovanna Nicodano, Loriana Pellizzon, Francesco Ravazzolo, Cesare Robotti, the editor, the anonymous reviewers of this paper, and the participants of the Eight Annual Society for Financial Econometrics (SoFiE) conference (Aarhus, Denmark, June 24-26, 2015), the Barcelona Graduate School of Economics Summer Forum (Barcelona, Spain, June 11-12, 2015), the Sixth Italian Congress of Econometrics and Empirical Economics (Salerno, Italy, January 21-23, 2015), the XV Workshop on Quantitative Finance (Florence, Italy, January 23-24, 2013), the Seventh Financial Risk International Forum (Paris, France, March 20-21, 2014), the workshop Measuring and Modeling Financial Risk with High Frequency Data (Florence, Italy, June 19-20, 2014), and at the seminars at Centre for Research in Econometric Analysis of Time Series (CREATES) (Aahrus, Denmark, 2014), the University of Maastricht (Maastricht, Netherlands, 2015) and the Security and Exchange Commission (Washington, DC, 2015), for useful discussions. All errors and omissions are our own. Massimiliano Caporin acknowledges financial support from the European Union, Seventh Framework Program FP7/2007-2013 under grant agreement SYRTO-SSH-2012-320270, and from Global Risk Institute in Financial Services and the Louis Bachelier Institute under the project Systemic Risk. Aleksey Kolokolov acknowledges financial support from the Riksbankens Jubileumsfond Grant Dnr: P13-1024:1, the VR Grant Dnr: 340-2013-5180, and the University of Padova grant CPDA 143827. Roberto Renò acknowledges financial support from Institute Europlace de Finance (EIF) under the research program A New Measure of Liquidity in Financial Markets. Email addresses: massimiliano.caporin@unipd.it (Massimiliano Caporin), alexeiuo@gmail.com (Aleksey Kolokolov), roberto.reno@univr.it (Roberto Renò)
} 


\section{Introduction}

Fig. 1 1 shows the intraday log-returns of four financial stocks on December 11, 2007. On that day, a Federal Open Market Commitee (FOMC) meeting ended with the decision to lower the target for the federal funds rate by 25 basis points, due to "slowing economic growth reflecting the intensification of the housing correction" and "financial strains", quoting the FOMC press release. The displayed financial stocks collapsed in the afternoon, with a contemporaneous log-return of approximately $-3 \%$, which is clearly visible in the figure. The figure also shows an evident increase, after the collapse, of both the stocks' volatility and their correlation. The Volatility Index (VIX) rose that day to 23.59 from 20.74 $(+13.7 \%)$. All industry portfolios in the next four days experienced a further large negative return, ranging from $-1.61 \%$ for utilities to $-4.90 \%$ for shops, with an average of $-3.25 \%$.

In the continuous-time literature, a price movement of $3 \%$ (when the local volatility is less than $0.5 \%$, thus of more than six standard deviations in volatility units) is typically modeled as a jump, that is, a discontinuous variation of the price process. There are three main routes to the detection of collective events such as those in Fig. 11 (1) detection of a jump in a portfolio that includes the stocks (e.g., the equity index), (2) detection of jumps in individual stocks, and (3) direct detection of the co-jump among the stocks. In the literature, much effort has been devoted to (1) and (2), both theoretically and empirically, but almost none to (3). This paper introduces a formal test for the detection of a simultaneous co-jump in a collection of stocks (or multi-jump, as we label it), argues that the third option is the most effective, and shows that it reveals additional economic information with systemic implications that could not be revealed by the first two.

Rare, dramatic multi-jumps can be interpreted as systemic co-jumps in the sense of Das and Uppal (2004); that is, as infrequent events that are highly correlated across a large number of assets 1 Co-jumps are correlated with market-wide information on economic fundamentals, with important consequences for asset allocation and risk management, as also recognized by the financial literature. For example, Longin and Solnik (2001) show that correlations increase after a collective crash in the market, dampening the diversification potential of portfolio managers, and Das and Uppal (2004) introduce a systemic jump component in their model to study its impact on portfolio choice. Bollerslev et al. (2008a) use common jumps to explain jumps in the aggregated market index and discuss that, for asset allocation, it is more important to be able to detect jumps occurring simultaneously among a large number of assets, because the effect of co-jumps in a pair of assets is negligible in a huge portfolio. Gilder et al. (2014) also study the relation between common jumps and jumps in the market portfolio, and they relate common jumps and news. The occurrence of co-jumps is also likely to affect the aggregate attitude to risk and thus has an impact on risk premia. For example, Bollerslev and Todorov (2011) empirically support the view that risk compensation due to large jumps is large and time-varying, and Drechsler and Yaron (2011) and Drechsler (2013) highlight the importance of transient non-Gaussian shocks to fundamentals in explaining the magnitude of risk premia. Our paper complements this evidence by showing that detected multi-jumps associated with bad news come together with large increases of the variance risk premium and a persistent increase of stock variances and correlations and are more likely followed by a decline in stock prices, while detected multi-jumps associated with good news imply a decrease in the

\footnotetext{
${ }^{1}$ The recent literature on systemic risk links the word "systemic" to the propagation of local shocks to the whole financial system, see e.g. Billio et al. (2012). The co-jumps we reveal in the data could also be interpreted as systemic in this sense, as they propagate from high-volume stocks to low-volume stocks (as shown in Section 4 ) and affect the price dynamics for a few days (for first moments, as shown in Section 5 or several months (for second order moments, as shown in Section 6 .
} 


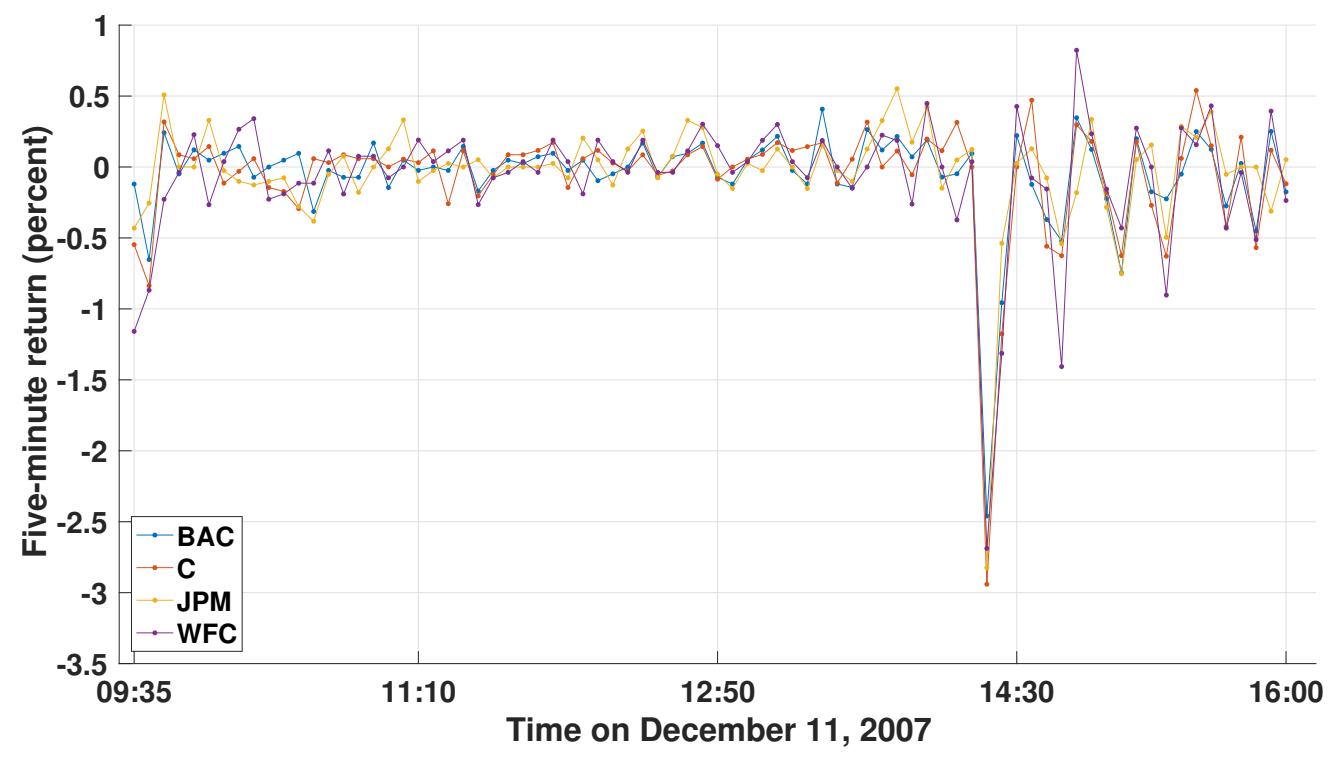

Figure 1: Intraday price changes (log-returns over five minutes) of Bank of America (BAC), Citigroup (C), JP Morgan (JPM), and Wells Fargo (WFC) on December 11, 2007. The price of all the four banking stocks rapidly declines around 14.10, during a Federal Open Market Committee meeting. After the event, both volatility and correlation among stocks increase.

variance risk premium and a persistent decrease in stock variances and correlations and are more likely followed by a rise in stock prices.

Despite the statistical, economic, and financial importance of systemic co-jumps, the literature is still missing a formal test to use as an effective tool for their detection. A vast literature concentrates on univariate jump tests applied to broad portfolios, such as stock indices, see, e.g., Barndorff-Nielsen and Shephard (2006); Andersen et al. (2007b); Lee and Mykland (2008); Jiang and Oomen (2008); AïtSahalia and Jacod (2009); Corsi et al. (2010). Progress on developing tests for common jumps in a pair of asset prices was started by Barndorff-Nielsen and Shephard (2003). They propose a way to separate out the continuous and co-jump parts of quadratic covariation of a pair of asset prices. Mancini and Gobbi (2012) develop an alternative threshold-based estimator of continuous covariation. Jacod and Todorov (2009) propose two tests for co-jumps, building on functionals that depend, asymptotically, on co-jumps only. Bandi and Renò (2016) propose a test for co-jumps based on nonparametric infinitesimal moments. Finally, Bibinger and Winkelmann (2015) develop a co-jump test using spectral methods. However, these methodologies apply to the case $N=2$ assets only, and their generalization to the case $N>2$ is not trivial. Bollerslev et al. (2008a) propose a test for common jumps in a large panel $(N \rightarrow \infty)$, which is based on the pairwise cross-product of intraday returns. In empirical works, the detection of multivariate jumps is typically achieved with a simple co-exceedance rule (see, e.g., Gilder et al., 2014), according to which the co-jump test is the intersection of univariate tests. We fill this gap in the literature by introducing a novel testing procedure for multi-jumps, that is, a simultaneous co-jump in a collection of assets, which naturally applies to the case $N \geq 2$, with $N$ finite. The proposed approach builds on the comparison of two types of suitably introduced smoothed power variations. High values of the test-statistics (which are asymptotically $\chi^{2}(N)$ under the null) signal the presence of a co-jump among stocks. The smoothing procedure depends on a bandwidth that can be interpreted as the ratio of the return to the local volatility above which the return is potentially considered a jump. 
Results from the new testing procedure applied to stock prices in the period 2003-2012 reveal the significant presence of multi-jumps in the data. However, when big news hit the market, not all the stocks appear to jump. The speed of reaction to news appears to be driven by trading volume, so that low-volume stocks underreact and are much slower in incorporating new information. For this reason, the test is more powerful when applied to a relatively small panel (we mainly use $N=20$ ) of high-volume stocks. The multi-jumps we detect in this smaller panel are rare but important events, which can be easily associated with relevant economic news whose effect is diffused in the market. This allows us to interpret them as systemic events as they propagate within the market from high-volume stocks to lower volume ones.

The detection of multi-jumps unveils additional information with respect to that conveyed by traditional univariate jump tests in the index, or even by univariate jump tests in portfolios composed of high-volume stocks only. We show that this is due to the fact that univariate jump statistics are substantially less powerful than the multi-jump test proposed here. For example, we provide evidence for 20 clear co-jumps in our sample, but only fewer than seven of these, in the best case, are also revealed by univariate test statistics based on the index. Univariate statistics applied to single time series of portfolio returns not only are affected by the slow incorporation of news of the low-volume stocks, but they are also jeopardized by the sudden spike in volatility typically associated with co-jumps. Our multi-jump test statistics are instead robust to these dynamic features of asset prices.

After the occurrence of a multi-jump, returns of individual stocks in the subsequent days tend to have the same sign of the return in the jump day. This unveils short-term predictability of stock returns induced by co-jumps. The effect is strong enough to overcome the notoriously large noise of daily returns, something that typically hampers attempts to assess predictability over short horizons. Predictability is again consistent with the underreaction story, that is the idea that news is incorporated into prices by trading volume and that market-wide news is slowly incorporated in low-volume stocks. Indeed, predictability is found to be significantly stronger for low-volume stocks.

Moreover, the multi-jumps we detect are strongly correlated with changes in the variance risk premium, with spikes being associated with bad news and declines being associated with good news. These results are in line with recent theoretical literature underscoring the impact of jumps in fundamentals on changes in aggregate risk aversion and, thus, in market prices, as well as with the empirical results in Todorov (2010), who makes use of a parametric model to show that price jumps are linked to the variation in the variance risk premium. When multi-jumps are used, the association with changes in the variance risk premium becomes clear-cut also in our fully nonparametric setting. Furthermore, we show that the occurrence of multi-jumps is strongly correlated with future stock variances and correlations, again with an intuitive pattern: bad news is associated with an increase in variances and correlations (thus confirming, on a formal statistical ground, the anecdotical evidence in Fig. 1), and good news is associated with a decline in both. The impact is persistent and lasts for a few months. This contrasts the typical finding of a transient impact of jumps found in the literature, which could be spuriously due to the poor power of univariate test-statistics shown here. All the empirical evidence thus is consistent in indicating that the multi-jumps we detect on stocks with high trading volume affect the dynamics of prices in the whole market.

Finally, using simulations of realistic price processes that accommodate the most relevant empirical features, we show that the proposed test is remarkably powerful in detecting multi-jumps, especially after controlling for partial adjustment effect theorized by standard microstructural models of price formation, 
and simulated to mimic the underreaction of less heavily traded stocks, and strongly outperforms the univariate tests in terms of power, without compromising its size.

The remainder of the paper is organized as follows. Section 2 describes the continuous-time jumpdiffusion model adopted in the paper. Section 3 explains the formal testing procedure and provides asymptotic results. Section 4 applies the test to real data, provides evidence for the presence of rare systemic co-jumps, and discusses the role of trading volume in incorporating information into prices. Section 5 illustrates the short-term predictability induced by multi-jumps, and Section 6 shows the implications for variance, correlations, and the variance risk premium. Section 7 presents results on simulated price dynamics. Section 8 concludes. An online appendix contains additional material.

\section{Model}

Denote the log-prices of an $N$-dimensional vector of assets by $X=\left(X^{(i)}\right)_{i=1, \ldots, N}$. We assume that stock prices evolve continuously on a filtered probability space $\left(\Omega, \mathcal{F},(\mathcal{F})_{t \in[0, T]}, \mathcal{P}\right)$ satisfying the usual conditions, and we assume the dynamics for $X$ specified as

$$
d X_{t}=a_{t} d t+\boldsymbol{\Sigma}_{t} d W_{t}+d J_{t}
$$

accommodating for continuous (through the Brownian motion component $W_{t}$ ) and discontinuous (through the jump component $J_{t}$ ) shocks. Details on the (mild) assumptions about the coefficients of the dynamics of Eq. (1) are provided in Assumption 2 in the case of finite activity jumps and in Assumption 3 in the more general case of infinite activity jumps (see Appendix A). The relevant case, in practice, is the first one because the theory is applied to detect economically significant joint variations of large magnitude.

The model encompasses virtually all parametric models typically used in financial applications. It allows each component of $X$ to include idiosyncratic jumps (that occur only for a single stock) as well as common jumps among stocks. It also allows for price and volatility multi-jumps, as the process $\boldsymbol{\Sigma}_{t}$ could also depend on the same $J_{t}$ appearing in the price equation. Define the process

$$
\Delta X_{t}=X_{t}-X_{t^{-}},
$$

and, as an example, consider the case $N=3$. The common jumps between the first two components, $X^{(1)}$ and $X^{(2)}$, can be rewritten as

$$
\Delta X_{t}^{(1)} \Delta X_{t}^{(2)}=\gamma_{t}^{1(2)} \gamma_{t}^{2(1)} \Delta N_{t}^{12}+\gamma_{t}^{1(23)} \gamma_{t}^{2(13)} \Delta N_{t}^{123}
$$

where $N^{12}$ and $N^{123}$ are independent counting processes and the notation $\gamma_{t}^{i(j k \ldots)}$ indicates the jump size at time $t$ of the $i$ th component when the $j$ th, $k$ th, ... are jumping jointly. Common jumps among the three components can be rewritten as

$$
\Delta X_{t}^{(1)} \Delta X_{t}^{(2)} \Delta X_{t}^{(3)}=\gamma_{t}^{1(23)} \gamma_{t}^{2(13)} \gamma_{t}^{3(21)} \Delta N_{t}^{123}
$$

The inference procedure is designed to test the null

$$
\sum_{0 \leq t \leq T} \Delta X_{t}^{(1)} \Delta X_{t}^{(2)} \Delta X_{t}^{(3)}=0
$$


against the alternative

$$
\sum_{0 \leq t \leq T} \Delta X_{t}^{(1)} \Delta X_{t}^{(2)} \Delta X_{t}^{(3)} \neq 0
$$

The presence of a multi-jump among three assets implies the presence of co-jumps between each pair of them (unless jump sizes add up to zero). However, the presence of co-jumps between each pair of assets does not necessarily imply the presence of a multi-jump among them.

We do not explicitly include in the model market microstructure contaminations, because the proposed method is thought to be applied at moderately low frequencies (e.g., five minutes), so that the impact of microstructure noise is negligible. In principle, the theory could be extended to include market microstructure noise by pre-averaging returns before applying smoothed estimators, as in Podolskij and Vetter (2009) and Hautsch and Podolskij (2013), or using spectral methods along the lines of Mancino and Sanfelici (2011) and Bibinger and Winkelmann (2015).

\section{Multi-jumps inference}

Assume to record $X$ in the interval $[0, T]$, with $T$ fixed, in the form of $n+1$ equally spaced observations and write $\Delta=T /\left.n\right|^{2}$ Define the evenly sampled logarithmic returns as

$$
\Delta_{j} X=X_{j \Delta}-X_{(j-1) \Delta}, \quad j=1, \ldots, n
$$

To formulate the statistical properties of the test, define the following sets:

$$
\begin{aligned}
\Omega_{T}^{M J, N} & =\left\{\omega \in \Omega \mid \text { the process } \prod_{j=1}^{N}\left(\Delta X^{(j)}\right)_{t} \text { is not identically } 0\right\} \\
\bar{\Omega}_{T}^{N} & =\Omega \backslash \Omega_{T}^{M J, N} .
\end{aligned}
$$

The set $\Omega_{T}^{M J, N}$ contains all the trajectories with multi-jumps among all $N$ assets in $[0, T]$. The complementary set $\bar{\Omega}_{T}^{N}$ contains trajectories without multi-jumps in $N$ stocks; it could, however, contain jumps and multi-jumps up to $N-1$ stocks. Testing for multi-jumps is equivalent to testing

$$
\mathcal{H}_{0}:\left(\left(X_{t}(\omega)\right)_{t \in[0, T]} \in \bar{\Omega}_{T}^{N}\right) \text { versus } \mathcal{H}_{1}:\left(\left(X_{t}(\omega)\right)_{t \in[0, T]} \in \Omega_{T}^{M J, N}\right) .
$$

Inference is based on two novel jump-robust integrated variance estimators, which are both called smoothed realized variance (SRV). They constitute a generalization, particularly suitable to our application, of the truncated realized variance estimator of Mancini (2009). The first one takes the form

$$
\operatorname{SRV}\left(X^{(i)}\right):=\sum_{j=1}^{n}\left|\Delta_{j} X^{(i)}\right|^{2} \cdot K\left(\frac{\Delta_{j} X^{(i)}}{H_{j \Delta, n}^{(i)}}\right),
$$

where $X^{(i)}$ and $H^{(i)}$ are the $i$ th components of the vectors $X$ and $H$ and $K(\cdot)$ and $H_{t, n}$ are the kernel

${ }^{2}$ The requirement of equally spaced observation can be easily relaxed to non-equally spaced observations, if we set $\bar{\Delta}=\max _{i=1, \ldots, n}\left(t_{i}-t_{i-1}\right)$, where $t_{i}$ are observation times, and require $\bar{\Delta} \rightarrow 0$, see Remark (i) of Theorem 4 in Mancini (2009). 
and bandwidth defined in Assumption 1. The bandwidth is written in the form

$$
H_{t, n}^{(i)}=h_{n} \cdot \widehat{\sigma}_{t}^{(i)} \sqrt{\frac{T}{n}},
$$

where $\widehat{\sigma}_{t}^{(i)}$ is a point estimator of the local standard deviation of the $i$ th stock, $i=1, \ldots, N$. We call $h_{n}$ the bandwidth parameter. Its role is to gauge the largeness of high-frequency returns with respect to the local volatility. For simplicity, we use the same $h_{n}$ across all stocks, which makes sense because we are normalizing with respect to each stock volatility. We use an automated criterion for its selection, described in Appendix B.1.

This estimator coincides with the integrated variance estimator in Mancini (2009) when $K(x)=$ $I_{\{|x| \leq 1\}}$. The intuition is then similar to that of Mancini $(2009)$ : smoothed squared returns $\left|\Delta_{j} X^{(i)}\right|^{2}$. $K\left(\Delta_{j} X^{(i)} / H_{j \Delta, n}^{(i)}\right)$ are close to squared returns $\left|\Delta_{j} X^{(i)}\right|^{2}$ when returns are small; they instead are close to zero when returns are large. The extent of largeness is gauged by the bandwidth $H_{j \Delta, n}$ (or by $h_{n}$, in local volatility units). Asymptotically, this procedure annihilates the jumps. The estimator of Mancini (2009) is the most draconian in this respect, because using the indicator function implies that smoothed returns are zero when returns are larger than $H_{j \Delta, n}$ (dubbed threshold in Mancini's terminology). The advantage of replacing the indicator function with a kernel is that it provides an estimator that depends smoothly on the bandwidth. This stabilizes the procedure in small samples (by making it less prone to type I and II errors due to erroneous bandwidth selection) and also eases bandwidth selection.

The second estimator takes the form

$$
\widetilde{\operatorname{SRV}}^{N}\left(X^{(i)}\right):=\sum_{j=1}^{n}\left|\Delta_{j} X^{(i)}\right|^{2} \cdot\left(K\left(\frac{\Delta_{j} X^{(i)}}{H_{j \Delta, n}^{(i)}}\right)+\prod_{k=1}^{N}\left(1-K\left(\frac{\Delta_{j} X^{(k)}}{H_{j \Delta, n}^{(k)}}\right)\right)\right) .
$$

Returns in Eq. 77 are smoothed twice. The first term, $K\left(\frac{\Delta_{j} X^{(i)}}{H_{j \Delta, n}^{(i)}}\right)$, has the same effect as in Eq. 5 . The second term, $\prod_{k=1}^{N}\left(1-K\left(\frac{\Delta_{j} X^{(k)}}{H_{j \Delta, n}^{(k)}}\right)\right)$, leaves the corresponding return similar to the original one also if all multivariate returns are big. Thus, even if, when $n \rightarrow \infty$, both smoothing procedures are meant to annihilate jumps, the smoothing in Eq. (7) allows the multi-jumps to survive. Theorems 2 and 3 in Appendix A provide the asymptotic limit for the two smoothed realized variance estimators and represent our theoretical base for inference and testing.

Our proposed test statistics is based on the difference between the two smoothed realized variance estimators. Such a difference tends to zero under the null and to a positive value under the alternative. However, we need to randomize one of them to obtain a non degenerate limit distribution under the null. We do so by borrowing from a wild bootstrap technique suggested in Podolskij and Ziggel (2010) and replacing SRV by SRRV (smoothed randomized realized variance), defined as

$$
\operatorname{SRRV}\left(X^{(i)}\right):=\sum_{j=1}^{n}\left|\Delta_{j} X^{(i)}\right|^{2} \cdot K\left(\frac{\Delta_{j} X^{(i)}}{H_{j \Delta, n}}\right) \cdot \eta_{j}^{i}, \quad i=1, \ldots, N
$$

where $\left(\eta_{j}^{i}\right)_{1 \leq i \leq N, 1 \leq j \leq n}$ is an $N \times n$ matrix of independent and identically distributed (i.i.d.) draws, defined on the canonical extension $\left(\Omega^{\prime}, \mathcal{F}^{\prime},\left(\mathcal{F}^{\prime}\right)_{t \in[0, T]}, \mathcal{P}^{\prime}\right)$ of the original probability space $\left(\Omega, \mathcal{F},(\mathcal{F})_{t \in[0, T]}, \mathcal{P}\right)$ and independent from $\mathcal{F}$, such that $\mathbf{E}\left[\eta_{j}^{i}\right]=1$ and $\operatorname{Var}\left[\eta_{j}^{i}\right]=V_{\eta}<\infty$. In our applications of the test, the $\eta_{j}^{i}$ are allowed to take the values $\{1+\tau, 1-\tau\}$ with equal probability, so that $V_{\eta}=\tau^{2}$. We use the small value of $\tau=0.05$ so that, in practice, SRRV is virtually indistinguishable 
from SRV. Nevertheless, its introduction has theoretical value in defining the test distribution under the null.

We write our proposed test statistics to detect multi-jumps as

$$
\mathrm{S}_{\mathrm{n}, \mathrm{N}}:=\frac{1}{V_{\eta}} \sum_{i=1}^{N} \frac{\left(\operatorname{SRRV}\left(X^{(i)}\right)-\widetilde{\operatorname{SRV}}^{N}\left(X^{(i)}\right)\right)^{2}}{\operatorname{SQ}\left(X^{(i)}\right)}
$$

where

$$
\mathrm{SQ}\left(X^{(i)}\right):=\sum_{j=1}^{n}\left|\Delta_{j} X^{(i)}\right|^{4} \cdot K^{2}\left(\frac{\Delta_{j} X^{(i)}}{H_{j \Delta, n}}\right), \quad i=1, \ldots, N .
$$

The asymptotic behavior of the $S_{n, N}$ statistics is described in Theorem 1 (proof in Appendix A).

Theorem 1. Under Assumptions 1 and 2, if $\left(\eta_{j}^{i}\right)_{1 \leq i \leq N, 1 \leq j \leq n}$ is pairwise independent, as $n \rightarrow \infty$, it holds that

$$
\left\{\begin{array}{ll}
\mathrm{S}_{\mathrm{n}, \mathrm{N}} \stackrel{d}{\longrightarrow} \chi^{2}(N), & \text { on } \bar{\Omega}_{T}^{N} \\
\mathrm{~S}_{\mathrm{n}, \mathrm{N}} \stackrel{p}{\longrightarrow}+\infty & \text { on } \Omega_{T}^{M J, N}
\end{array} ;\right.
$$

where $\chi^{2}(N)$ denotes the $\chi$-square distribution with $N$ degrees of freedom.

Theorem 1 implies that the statistic $S_{n, N}$ can be used for testing for the presence of multi-jumps. Under $\mathcal{H}_{0}$, the value of $\mathrm{S}_{\mathrm{n}, \mathrm{N}}$ is distributed as a $\chi^{2}$ with $N$ degrees of freedom. Under $\mathcal{H}_{1}$, that is, in the presence of multi-jumps, it diverges as the number of observations $n$ increases.

The test defined in Eq. (9) does not require the estimation of the covariance matrix of stocks returns. This compares favorably with our test with respect to alternatives employing covariance matrix estimates, both in terms of robustness and ease of computation.

\section{Testing for systemic co-jumps}

The data set we use is the collection of $N=442$ stocks belonging to the Standard \& Poor 500 (S\&P 500) index and quoted on the New York Stock Exchange. We include S\&P 500 constituents in June 2012, which were traded for at least five years in the sample. The sample ranges from January 2, 2003 to June 29, 2012, for a total of 2,392 trading days, and consists of one-minute returns from 9:30 to 16:00, New York Greenwich Mean Time. The data were recovered from Kibot.com and they went through a standard filtering procedure. Kibot retrieves data from multiple exchanges and electronic networks. Data are cross-checked, tested, and verified so that outliers and bad ticks are removed. To detect multi-jumps, we use five-minute grids, as explained in Appendix B. The five-minute frequency represents a trade-off between achieving enough statistical power and avoiding distortions that could arise from microstructure noise. For fair comparison with the individual stocks in some applications, we create a built-in portfolio, labeled S\&P 442, using the weighted average of the 442 stock returns using weights proportional to the average trading volume in the sample. The correlation of the returns of the S\&P 442 portfolio and the benchmark S\&P 500 cash index (computed by Standard \& Poor's weighting with market capitalization) is $97.62 \%$. For bandwidth selection, we use the thumb rule $h_{N}=h_{0}+\frac{c}{N}$, with $c=9.57$ (calibrated as described in Appendix B and $N$ is the number of stocks employed in the test. We use three bandwidth parameters: $h_{0}=0.9$ (small bandwidth), $h_{0}=1.15$ (intermediate bandwidth), and $h_{0}=1.4$ (large bandwidth). 
We detect multi-jumps each day across $N$ stocks, with $N \leq 442$. We use a confidence interval $1-\alpha^{*}$ such that the expected number of false positives in the sample is 0.1 asymptotically; that is $\alpha^{*}=4.18 \cdot 10^{-5}$. The simulation study in Subsection 7.2 makes clear that using all available stocks does not necessarily maximize power. This reflects the way the test procedure is constructed. As $N$ increases, power initially increases, because the test is based on multiplication of kernel-convoluted returns, which implies that the test-statistics get exponentially larger when returns at the jump time are large. However, as $N$ increases, the likelihood of including stocks with a small return at the multi-jump time increases. This trade-off originates the hump-shaped behavior of the power as a function of the number of stocks selected, displayed in Fig. 13 for simulated data. Clearly, power is maximized if we select the $N$ corresponding to the top of the peak. The simulation evidence also confirms that the number of false positives detected by our test at the confidence interval $\alpha^{*}$, when using the large bandwidth, is negligible, so that we can interpret virtually all the multi-jumps detected in the data at that confidence interval (at least those with the large bandwidth) as true signal. This provides guidance not only on how many stocks it is convenient to include, but also on how to select the stocks to include. This is what we do in what follows.

Fig. 2 is then crucial in this respect, as it shows the number of detected multi-jumps at the confidence interval $\alpha^{*}$ as a function of the number of stocks $N$. The four panels correspond to four different stock selection criteria: (1) picking stocks randomly (the shown result is just one draw), (2) picking stocks with higher $\beta$ first, with $\beta$ s computed from daily close-to-close return of individual stocks and the S\&P 500 cash index, (3) picking stocks with higher average trading volume first, and (4) picking stocks with lower illiquidity first, using the Amihud (2002) measure for each stock, computed as the average of the absolute value of the open-to-close return divided by daily traded volume. For both volume and illiquidity measures, we use moving averages with a window of two hundred days.

The test detects more multi-jumps, in all cases, when we use a number of stocks roughly between 10 and 50. As discussed above, this implies that the test is more powerful in this range. Among different selection criteria, high trading volume is the most effective way to select the stocks to be included in the computation of the test. Amihud's illiquidity measure and stock $\beta$ are instead not as effective, being just slightly more powerful than sorting randomly. Using the 20 stocks with higher trading volume, we detect 20 multi-jumps with the large bandwidth, 42 with the intermediate bandwidth, and 101 with the small bandwidth. Thus, detected multi-jumps are largely statistically significant in our sample (under the null, we expect just 0.1 false positives in our sample), even if they are still rare events. However, these rare events are also strongly economically significant. Table 1 reports the dates of the 20 days detected by the large bandwidth and associates macroeconomic and financial information to selected dates. It also reports the return of the equally weighted portfolio of the 20 stocks at the instant of the multijump, the time of the multi-jump, the corresponding VIX daily changes, S\&P 500 percentage change and percentage volume (aggregated over all 20 stocks) changes. Many of the multi-jumps in Table 1 can be easily associated with impactful economic news, mainly related to Federal Reserve activity, more prominently FOMC meetings. The change in the S\&P 500 index is typically of the same sign as the multi-jump return, with few exceptions. Moreover, the average traded volume tends to be considerably higher (than the previous day) on days in which a multi-jump occurs. The VIX tends to move, in multi-jump days, in the opposite direction with respect to the market, as also noticed in Todorov and Tauchen (2011) (below we provide formal statistical evidence of a significant increase of the variance premium associated with multi-jumps). Thus, these multi-jump events can be considered as a systemic consequence of market-wide news hitting the market. 
Panel A: Randomly selected

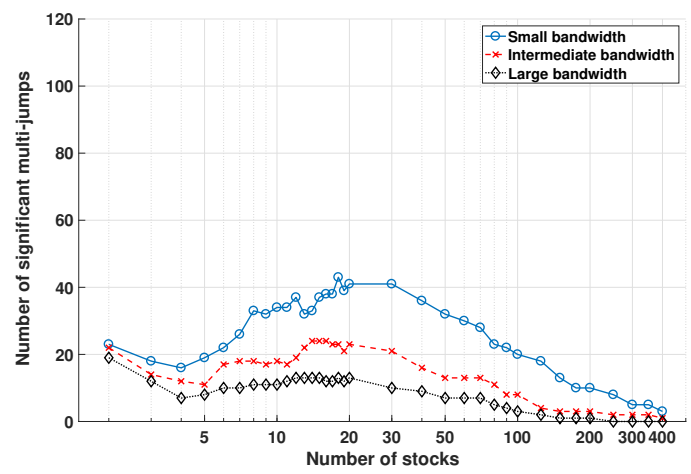

Panel C: Ranked with liquidity

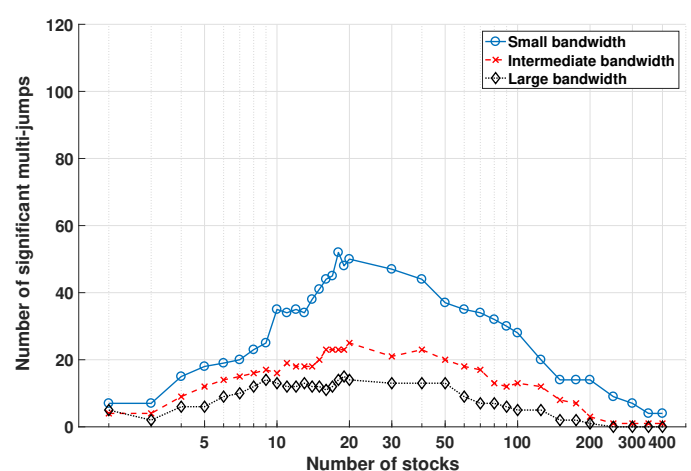

$\underline{\text { Panel B: Ranked with betas }}$

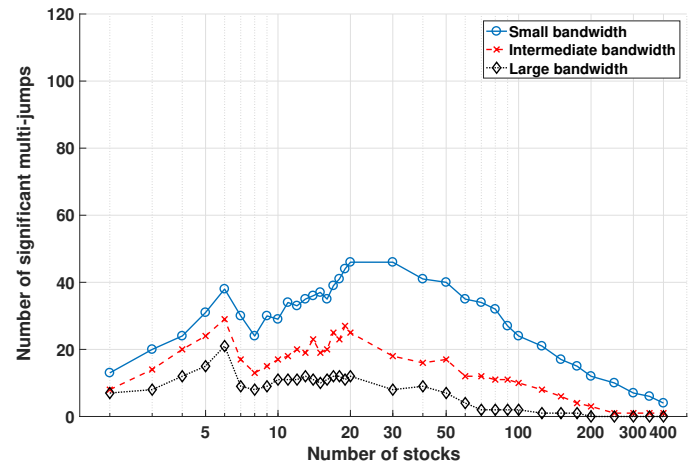

Panel D: Ranked with trading volume

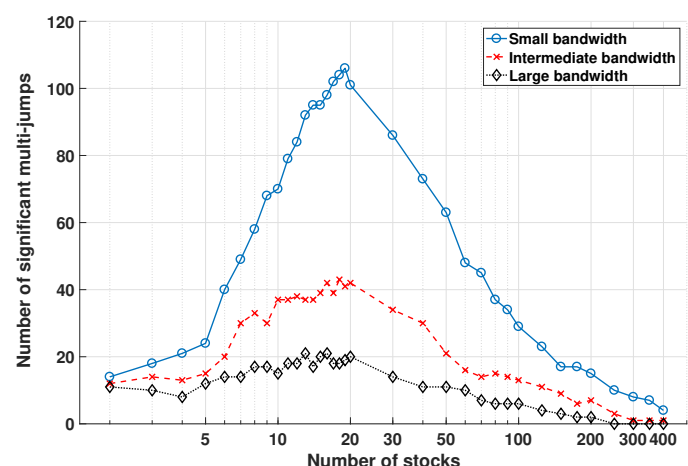

Figure 2: Number of significant multi-jumps for three different bandwidths, as a function of the number of stocks $N$ used in the test. Panel A covers random selection; Panel B, higher $\beta$ stocks first; Panel C, lower Amihud's price impact measure first; and Panel D, high-volume stocks first. The figure shows that the multi-jump test is more effective when an intermediate number of stocks is used and that the power of the test is much larger when using stocks ranked according to traded volume.

Why do stocks with high trading volume appear to jump more? To answer this question, we start by looking at an iconic day, that is, September 29, 2008, in the aftermath of the Lehman Brothers crisis. Fig. 3 shows the price dynamics of the S\&P 500 cash index, as well as that of portfolios composed of high- and low-volume stocks and portfolios composed of high- and low-beta stocks that afternoon. The figure makes clear that, as the market began to collapse, high-volume stocks reacted sooner and deeper, while low-volume stocks underreacted and moved with substantial delay and less impact. The S\&P 500 index, which is an average of high- and low-volume stocks, hovers in the middle. Low-beta stocks and high-beta stocks move similarly to low-volume and high-volume stocks that day. However, in general, sorting with trading volume is more effective than sorting with $\beta$ (Fig. 2) 3

Fig. 3 and the evidence on multi-jumps above sugges that "it takes volume to jump". Not all stocks react contemporaneously to big news, with stocks more actively traded being faster to react to news. Information flows to prices through volume. This idea is consistent with microstructural models of price formation, in which the traded price adjusts partially to changes in the efficient price (Amihud and Mendelson, 1987, Hasbrouck and Ho, 1987), reflecting learning of market makers about fundamentals. According to this model (which is simulated in Subsection 7.2 to mimic this effect), when there is a large shock in the efficient price, the traded price reacts with a delay that depends on the velocity of learning.

\footnotetext{
${ }^{3}$ Across stocks, the correlation between logarithmic average trading volume and beta is $12.51 \%$.
} 
Table 1: Detected multi-jumps. This table presents dates in which the multi-jump test (implemented with the large bandwidth on the 20 stocks with the highest trading volume) rejects the null at the confidence level $\alpha^{*}=4.18 \cdot 10^{-5}$, listed with the five-minute return of the equally weighted portfolio of the 20 stocks at the instant of the multi-jump, multijump time, percentage daily change in S\&P 500, percentage daily volume change, VIX daily differences, and economic and financial events occurring on the same day.

\begin{tabular}{|c|c|c|c|c|c|c|}
\hline Date & $\begin{array}{l}\text { Multi- } \\
\text { jump } \\
\text { five- } \\
\text { minute } \\
\text { return } \\
\text { (percent) }\end{array}$ & $\begin{array}{l}\text { Multi- } \\
\text { jump } \\
\text { time }\end{array}$ & $\begin{array}{l}\text { S\&P } 500 \\
\text { daily } \\
\text { change } \\
\text { (per- } \\
\text { cent) }\end{array}$ & $\begin{array}{l}\text { Volume } \\
\text { daily } \\
\text { change } \\
\text { (per- } \\
\text { cent) }\end{array}$ & $\begin{array}{l}\text { VIX } \\
\text { daily } \\
\text { change }\end{array}$ & Economic and financial events \\
\hline $19-F e b-2004$ & -0.56 & $15: 25$ & -0.41 & +19.47 & +0.21 & \\
\hline 01-Sep-2004 & +0.52 & $13: 10$ & +0.15 & +14.39 & -0.38 & \\
\hline 07-Jun-2005 & -0.52 & 14:05 & -0.02 & +26.30 & +0.11 & \\
\hline 31-May-2006 & -0.70 & $14: 10$ & +0.81 & +12.88 & -2.22 & May 10, FOMC minutes released \\
\hline 29-Jun-2006 & +0.68 & $14: 15$ & +2.16 & +18.39 & -2.76 & FOMC statement \\
\hline 08-Aug-2006 & -0.96 & $14: 20$ & -0.34 & +29.23 & -0.00 & FOMC keeps its target for the federal funds rates \\
\hline 18-Sep-2007 & +1.24 & $14: 10$ & +2.92 & +30.98 & -6.13 & FOMC lowers target for federal funds rates $(50 \mathrm{bps})$ \\
\hline 11-Dec-2007 & -1.58 & $14: 10$ & -2.53 & +33.37 & +2.85 & FOMC lowers target for federal funds rates ( $25 \mathrm{bps})$ \\
\hline 29-Sep-2008 & -3.99 & $13: 40$ & -8.81 & +15.37 & +11.98 & FOMC meeting unscheduled \\
\hline 06-May-2010 & -4.47 & $14: 40$ & -3.24 & +49.28 & +7.89 & The Flash Crash \\
\hline 26-May-2010 & -1.17 & $15: 20$ & -0.57 & -9.83 & +0.41 & Minutes of board discount rate meetings (May, 25) \\
\hline 28-May-2010 & -0.83 & $12: 35$ & -1.24 & -7.66 & +2.39 & FED announces three small auctions \\
\hline 23-Jun-2010 & +0.75 & $14: 25$ & -0.30 & +8.36 & -0.14 & FOMC statement \\
\hline 10-Aug-2010 & +0.58 & $14: 25$ & -0.60 & -14.64 & +0.23 & FOMC statement \\
\hline 01-Sep-2010 & +1.00 & $09: 55$ & +2.95 & +13.44 & -2.16 & Minutes of FOMC (August, 31) \\
\hline 03-Nov-2010 & -0.61 & $14: 25$ & +0.37 & +11.36 & -2.01 & FOMC statement \\
\hline 23-Jun-2011 & +0.84 & $14: 55$ & -0.28 & +32.84 & +0.77 & FOMC meetings (June, 21 and 22 ) \\
\hline 01-Nov-2011 & +0.98 & $13: 25$ & -2.79 & +34.22 & +4.81 & \\
\hline 07-Nov-2011 & +0.60 & $13: 50$ & +0.63 & -17.84 & -0.31 & \\
\hline 14-Jun-2012 & +0.94 & $15: 00$ & +1.08 & -3.91 & -2.59 & \\
\hline
\end{tabular}

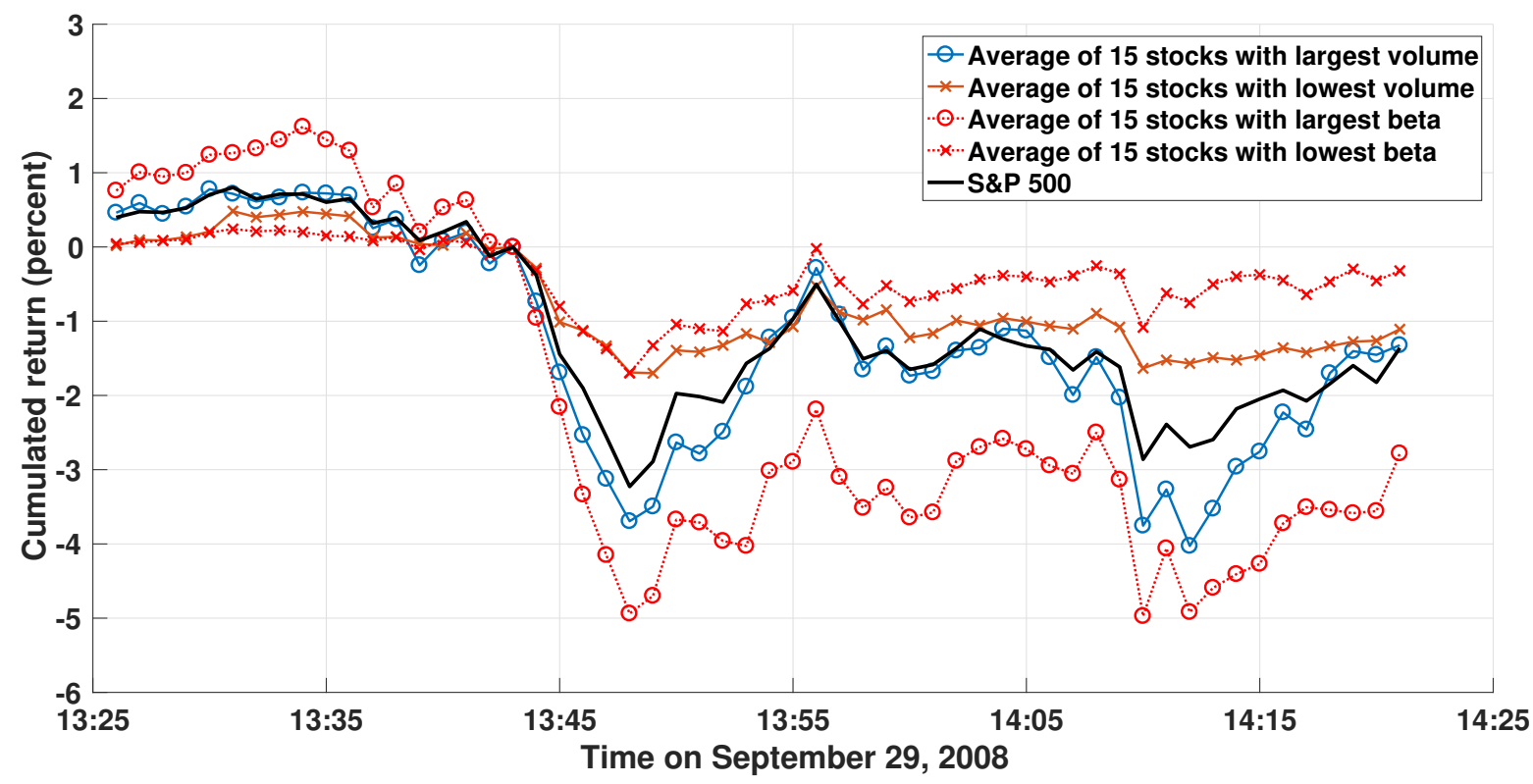

Figure 3: Price dynamics of selected portfolios during the market crash on September 29, 2008, in the aftermath of the Lehman Brothers default. Portfolios composed of stock with higher trading volume appear to react faster to news.

Our results suggest that the speed of learning is driven by trading volume. Thus, stocks with higher trading volume are faster in incorporating the unexpected news, and appear to jump more. Stocks with lower trading volume react with more delay and appear to jump less.

Table 1 provides evidence of increased aggregate volume on multi-jump days. This fact holds for individual stocks as well. To show this, we compute the average change in the logarithmic volume in 


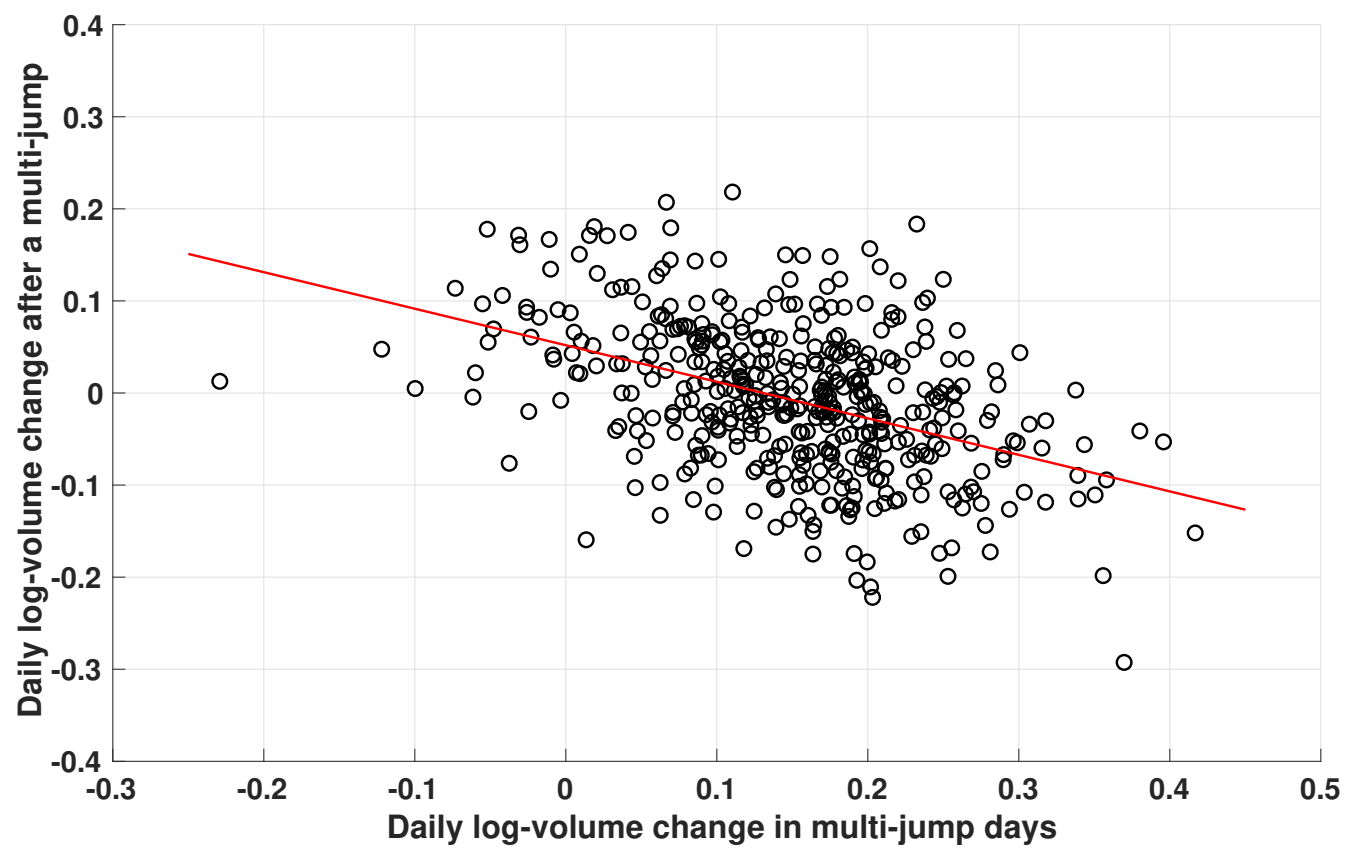

Figure 4: The relation between average change in logarithmic volume in multi-jump days and average change in logarithmic volume in days after multi-jumps for individual stock. The line is estimated from regression [12. The figure shows that trading volume tends to be higher in multi-jump days for individual stocks. Moreover, when the volume change is above average, volume drops the following day. When it is below average, volume still rises the next day. This is consistent with information flowing to prices through volume.

multi-jump days, with respect to the previous day and the average change in the logarithmic volume the day after multi-jumps, with respect to the multi-jump days. We do so for the 20 multi-jump days shown in Table 1 and for each of the 442 stocks in our sample. Fig. 4 shows the scatter plot of these two consecutive average log-volume changes and reveals two important things. First, in multi-jump days, trading volume tends to increase for almost all stocks. Second, stocks with a large change in volume in multi-jump days experience, on average, a drop in volume the following day and stocks with a small change in volume in multi-jump days experience, on average, an increase in volume the following day. The effect is strongly statistically significant. Denoting by $<\Delta \log V_{M J}>_{i}$ the average change in logarithmic volume on multi-jump days for stock $i$, and by $<\Delta \log V_{M J+1}>_{i}$ the same quantity the next day, regression estimates yield

$$
<\Delta \log V_{M J+1}>_{i}=\underset{(7.51)}{0.0518^{* * *}}-\underset{(10.04)}{0.3968^{* * *}} \cdot<\Delta \log V_{M J}>_{i}+\epsilon_{i}, \quad i=1, \ldots, 442,
$$

with T-statistics in parentheses and the corresponding regression line shown in Fig. 4 .

This finding corroborates the view that volume brings information into prices, so that stocks with high volume react faster to news, jump more, and then have a lower volume the next day, and stocks with low trading volume need more time to react to news, so that their volume increases the following day. This is the reason why using high-volume stocks is more powerful for jump detection. Thus, in what follows, we use the $N=20$ highest volume stocks to detect multi-jumps. 


\subsection{Comparison with univariate tests}

Even if a multi-jump in a panel of stocks implies a jump in any portfolio containing these stocks, traditional univariate jump statistics applied to a single time series of portfolio returns have a hard time in detecting these jumps. To show this, we compare the performance of the multi-jump test with that of the CPR and the BNS test (see Appendix B.2 for their description) in Fig. 5. The figure shows the scatter plot between the CPR test (panels A and C), and the BNS test (panels B and D), and the multijump test in the same days using the large bandwidth. Panels A and B report the CPR and BNS test in the same portfolio used for the multi-jump test, composed of the 20 highest volume stocks. Results are striking. While the multi-jump test is clearly powerful in separating signal from noise (we had to use a logarithmic scale for it), the univariate tests are not. Using the common confidence interval $\alpha^{*}$, the multi-jump test identifies 20 events (those listed in Table 1). The CPR test identifies only four events; the BNS test only one, namely September 18, 2007. The single event identified by CPR but not by the multi-jump test is February 28, 2006, in which the Google stock dropped $-8.24 \%$ (open-to-close), but the other stocks did not jump as such, with S\&P 500 moving only $-1.05 \%$ and our S\&P 442 replica moving $-0.96 \%$. So, the jump can be ascribed mainly to a single stock and, for this reason, the multi-jump test does not detect it.

Panels C and D show the CPR and BNS test on the S\&P 442 portfolio, which is what is commonly done by the empirical literature, e.g., testing on a portfolio made by a large number of stocks (typically, S\&P 500, for which results, not reported here, are virtually unchanged). With respect to the test in the high-volume portfolio, the statistics get lower, making jump detection even more difficult. CPR now reveals seven jumps; BNS, still one. Univariate tests are worringly low on clear multi-jump days, such as the collapse of September 29, 2008, whose magnitude is visible in Fig. 3. These results show the substantial inability of the considered univariate jump tests in capturing systemic events, neither in the index portfolio nor in the portfolio composed by a small number of high-volume constituents 4

The inability of univariate tests to detect largely visible jumps can be explained by the simulation evidence provided in Subsection 7.1. where we show that univariate tests are jeopardized by a contemporaneous increase in the volatility of volatility of the stocks or by a price/volatility co-jump, with both these events being likely to occur in turbulent days. The fact that low-volume stocks take more time to incorporate news makes the task of univariate tests even harder to accomplish. Our negative results on the power of univariate tests complement those of Bajgrowicz et al. (2016), who attribute up to 90\% of jump detections by univariate test statistics to spurious detection of volatility bursts. Thus, even if a co-jump in the stocks always implies a jump in the portfolio composed of these stocks, direct detection of the multi-jump with our test is much more powerful, especially when applied to large-volume stocks. The superiority of our testing procedure allows us to reveal the presence of simultaneous, non diversifiable co-jumps that Bajgrowicz et al. (2016) could not reveal using univariate tests.

Finally, the strong empirical evidence for the presence of rare, but impactful, co-jumps in the stock

\footnotetext{
${ }^{4}$ In the existing empirical literature, which finds substantially more jumps, what is typically done is implementing the test on a single five-minute grid. We instead apply the test on all the five possible grids that can be aggregated from one-minute returns and average the outcomes. On single grids, the CPR test finds from 18 to 30 jumps (depending on the grid) in the portfolio of high-volume stocks and from 26 to 38 jumps in the S\&P 442 index, BNS test finds from ten to 19 jumps in the portfolio of high-volume stocks and from seven to 17 jumps in the S\&P 442 index. We interpret the drop in the number of detected jumps when aggregating as the fact that these events are mostly due to size distortion (see also Bajgrowicz et al. 2016), as the aggregated test is more precise. The aggregation of the test on the five grids instead does not compromise the statistical power of the multi-jump test.
} 
Panel A: Comparison with the CPR test ( $N=20$ high-volume stocks $)$

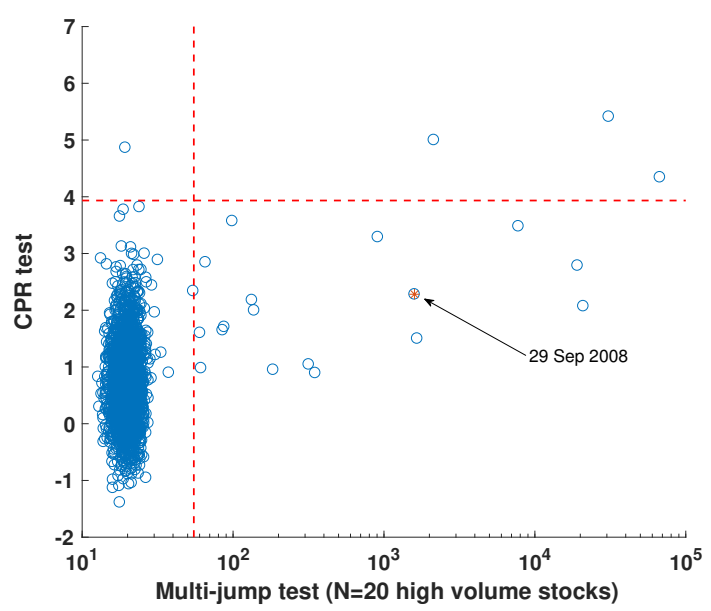

Panel C: Comparison with the CPR test (S\&P 442 index)

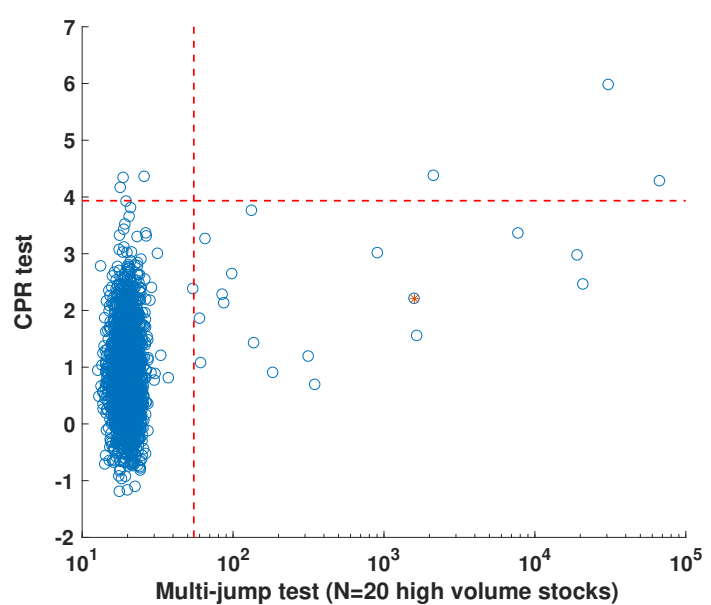

Panel B: Comparison with the BNS test $(N=20$ high-volume stocks $)$

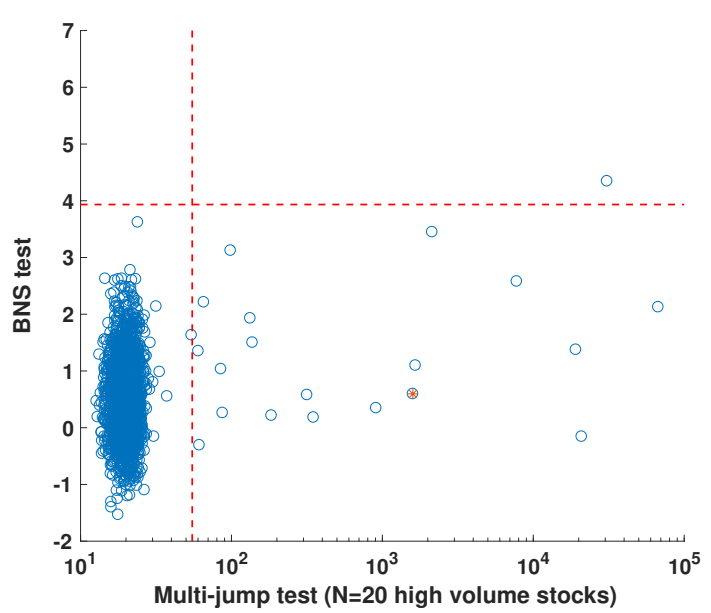

Panel D: Comparison with the BNS test (S\&P 442 index)

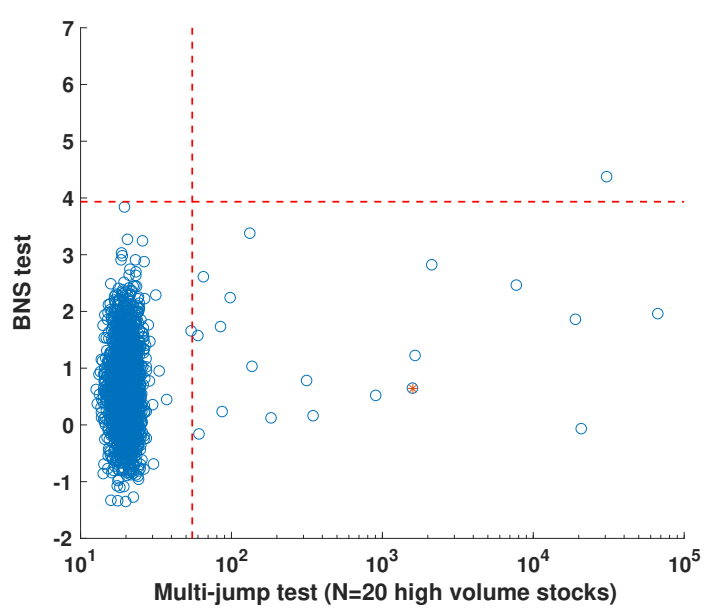

Figure 5: Comparison between the multi-jump test and traditional univariate tests. This figure presents scatter plots of the multi-jump test (large bandwidth, $N=20$ stocks with highest volume) and univariate tests (CPR, panels A and C, and BNS, panels B and D) applied to the equally weighted portfolio of the 20 highest volume stocks (panels A and B) and to the S\&P 442 index (panels C and D). Dashed lines are theoretical quantiles of the two tests at the confidence interval $\alpha^{*}$. While the multi-jump test separates noise from signal in a clear-cut way, univariate tests are less able to do so and fail to detect important and largely visible systemic jumps.

market can be reconciled with the results in Christensen et al. (2014), who instead find that most of the jumps in their sample tend to appear as very sharp continuous movements realizing over a very short time, much shorter than five minutes, suggesting that there are virtually no jumps in asset prices. Their empirical finding is puzzling because the theory (see, e.g., Back, 1991) allows for the presence of discontinuities in the price dynamics. Why are these discontinuities hardly seen in traded prices? Again, we can learn from models implying a delay in incorporating information, which depends on how actively traded the stock is. This delay can be explained by market microstructure models of price formation with partial adjustment, such as those in Hasbrouck and Ho (1987) and Amihud and Mendelson (1987), which imply that the traded price lags behind the fundamental price due to noise, liquidity needs, delayed price discovery, and so on. If the discontinuity is in the fundamental price, due to big news, it 
then materializes in the form of an apparently continuous movement of the traded price, explaining the findings of Christensen et al. (2014). At a moderate frequency, the discontinuity in the efficient price can still be revealed using jump tests, so that the two approaches are not necessarily in contradiction. The distinction between sharp continuous movements and jumps remains theoretically relevant, because it questions the data generating process of price formation, especially at a microstructural level, i.e., at much higher frequencies that those used here. However, the empirical question that is relevant is whether jumps are present in the efficient price dynamics, which we answer in the affirmative.

\section{Jump-induced short-term predictability}

Detected multi-jumps typically tend to be followed by returns with the same sign. This short-term predictability is illustrated by both panels of Fig. 6. Panels A and C show the distribution across the 442 stocks of daily stock returns in days immediately following the day in which the multi-jumps in Table 1 occurred. We distinguish between bad and good news, using the sign of the daily return on the S\&P 442 portfolio in the day of the multi-jump. The figure clearly shows that, after a multi-jump associated with bad news, stock returns are more likely to be negative and, after a multi-jump associated with good news, stock returns are more likely to be positive, with less variability. The difference in the variance of the two distributions can be explained by the occurrence of a jump in volatility, which is more likely with negative jumps, as shown in Bandi and Renò (2016). Panels D and F display average stock returns in days following multi-jumps, again divided for good and bad news, and show that the effect is significant on average. The effect is transient, so that it is dissipated after a few days and more volatile after bad news. Fig. 6 also shows that if we use the 20 days with the largest BNS or CPR test statistics applied to the stock index (instead of the 20 days with the largest multi-jump test statistics), the effect disappears, pointing out the inability of univariate jump tests to identify systemic events.

Short-term predictability driven by systemic co-jumps is compatible with the volume effect shown earlier. When big news with systemic implications impacts the market, stocks incorporate the news in the price at a rate that depends on trading volume. Low-volume stocks take more time to incorporate the information, so that the effect of the news could be still visible a long time after the arrival of the news itself. Because most of the multi-jumps occur in the afternoon, the effect can even propagate to the next day. Trading volume is higher for low-volume stocks in the day after the multi-jumps occur, suggesting incorporation of news is still taking place the next day for these stocks. To confirm this, Fig. 7 uses the same quantities of Fig. 6 for the most liquid (top 5\% average trading volume) and the most illiquid (bottom $5 \%$ average trading volume) stocks in our sample, showing that predictability is stronger for low-volume stocks, in particular after bad news.

To verify that predictability is stronger for low-volume stocks more formally, we again use a simple regression model. Denote by $\left\langle\log V>_{i}\right.$ the median (across days) logarithmic trading volume of stock $i$ and by $\mu_{\log V}$ and $\sigma_{\log V}$ their cross-sectional average and standard deviation. We define

$$
\overline{\log V}_{i}=\frac{<\log V>_{i}-\mu_{\log V}}{\sigma_{\log V}}
$$

as the standardized logarithmic trading volume for stock $i$. Thus, a positive value of $\overline{\log }_{i}$ is associated with a stock with a trading volume above median; a negative value, to a stock below median. Next, we define by $r_{i}^{M J^{-}}$and $r_{i}^{M J^{+}}$the median daily return of stock $i$ realized after the occurrence of a multi-jump associated with negative and positive news, respectively. Regression estimates (with $\epsilon_{t}$ representing a 
Panel A: Daily return after a multi-jump

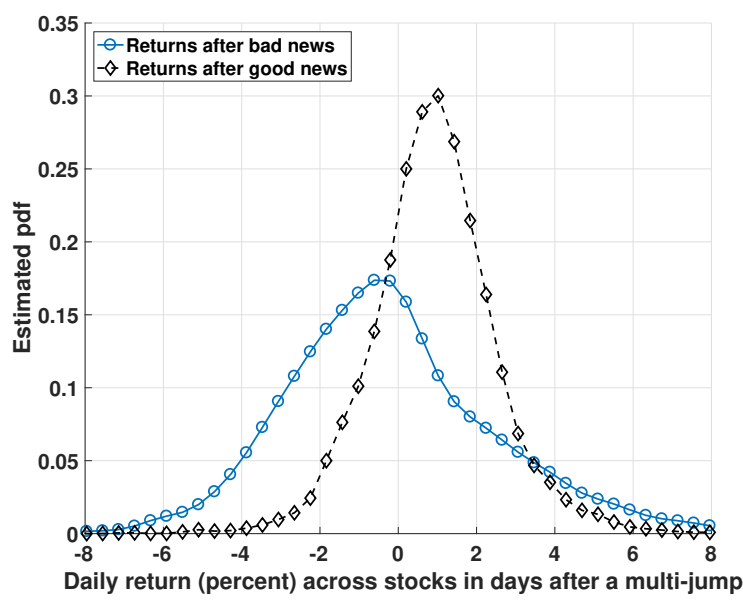

Panel B: Daily return after a BNS jump in S\&P 500

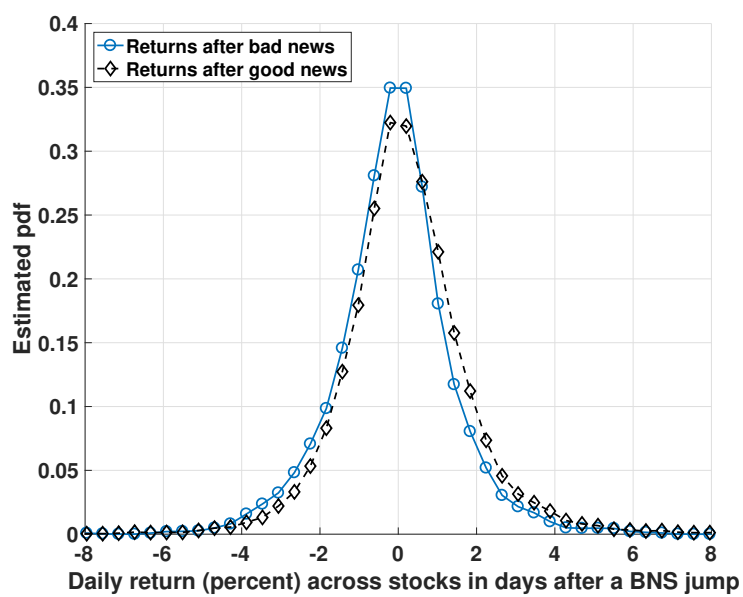

Panel C: Daily return after a CPR jump in S\&P 500

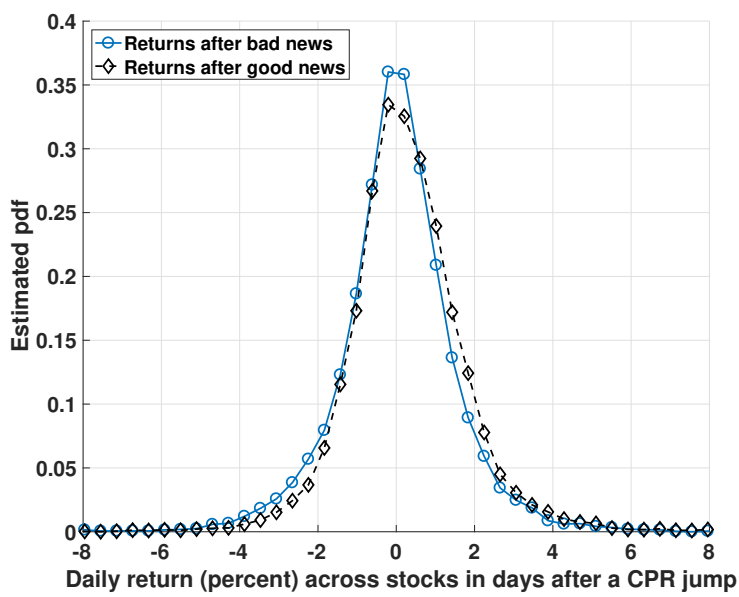

Panel D: Median return after a multi-jump

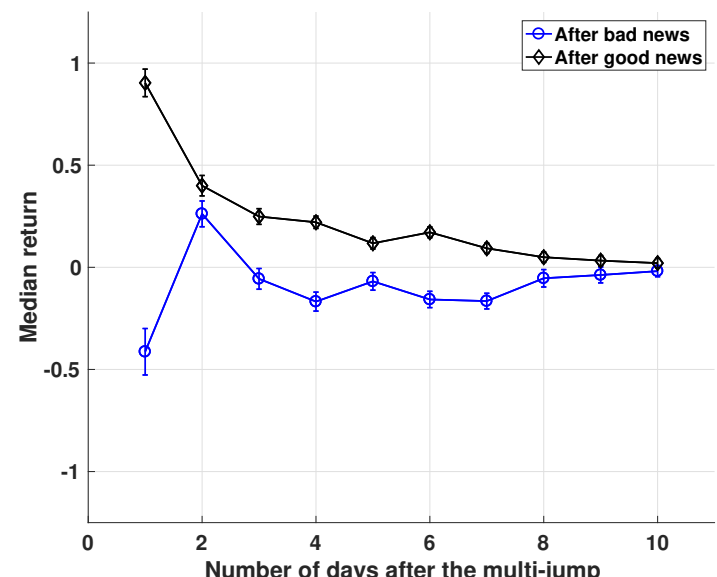

Panel E: Median return after a BNS jump in S\&P 500

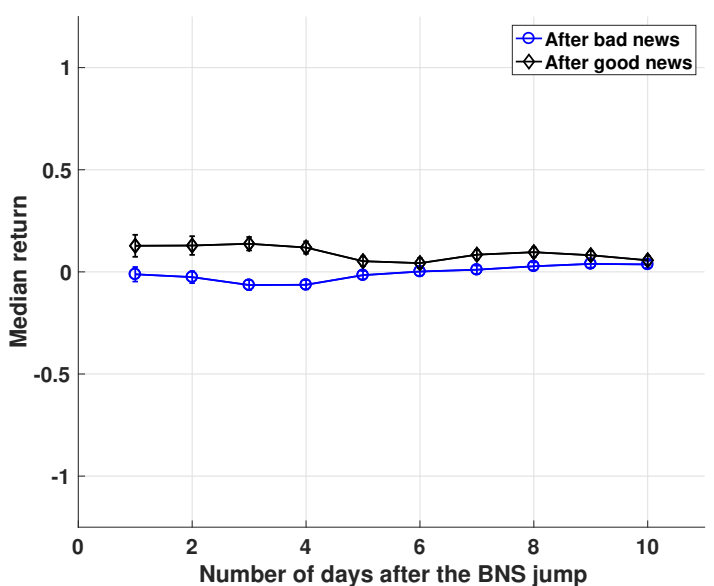

Panel F: Median return after a CPR jump in S\&P 500

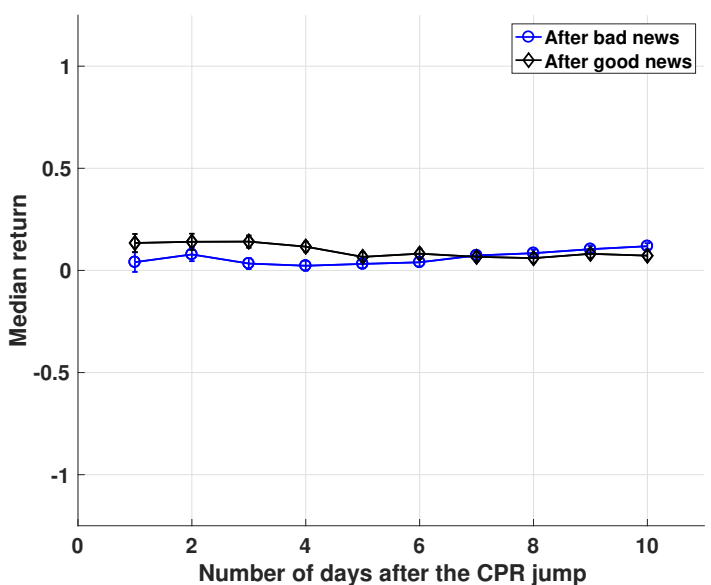

Figure 6: The jump-induced predictability effect. Panel A shows the distribution of pooled stock returns in the day following the occurrence of multi-jumps in Table 1 separated between good and bad news. Panel D shows the average cumulative stock return as a function of the days elapsed after the occurrence of the multi-jump, again separated between good and bad news. The figure shows a clear predictability effect, so that after a multi-jump the sign in stock returns tends to be the same as that of the multi-jump day. The effect is significant, but transient, as it disappears after a few days and is more volatile after bad news. Panels B, C and E, F show the same quantities when using the 20 days with the largest BNS and CPR test applied to the stock index. They show that univariate tests are unable to capture jump-induced predictability. (pdf = probability density function). 
Panel A: Pooled stock return distributions

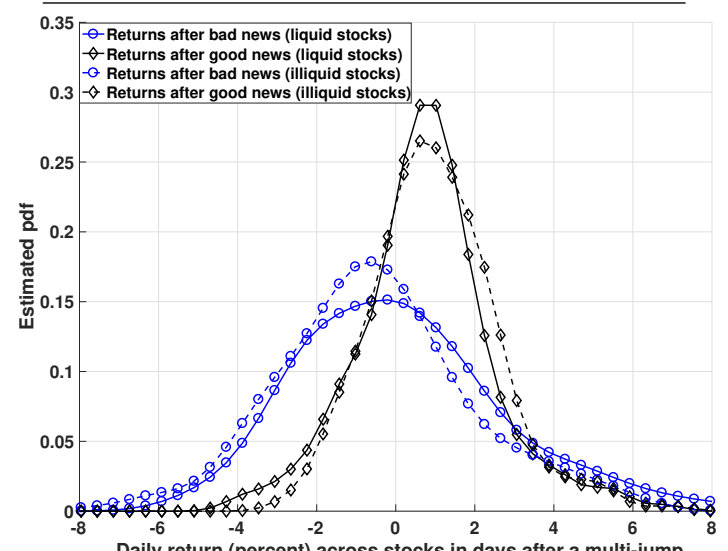

Panel B: Average stock returns

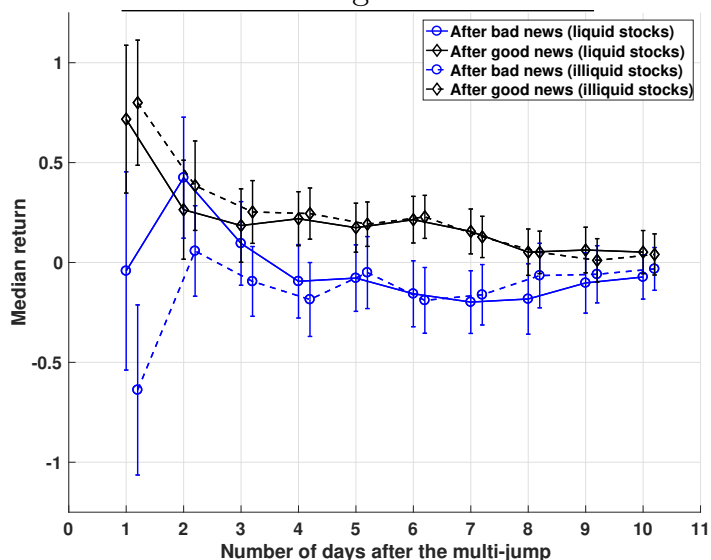

Figure 7: The jump-induced predictability effect for most liquid stocks (top 5\% average trading volume) and most illiquid stocks (bottom 5\% average trading volume) in our sample. Panel A shows the distribution of pooled stock returns in the day following the occurrence of multi-jumps listed in Table 1 separated between good and bad news. Panel B shows the average cumulative stock return as a function of the days elapsed after the occurrence of the multi-jump, again separated between good and bad news. The figure shows that predictability is stronger for illiquid stocks, especially after bad news.

noise with zero mean and variance $\sigma_{\epsilon}^{2}$ ) yield

$$
r_{i}^{M J^{-}}=\underset{(-13.9)}{0.4641^{* * *}}+\underset{(2.73)}{0.0914^{* * *}} \cdot \overline{\log V}_{i}+\epsilon_{i}, \quad i=1, \ldots, 442
$$

and

$$
r_{i}^{M J^{+}}=\underset{(36.7)}{0.9565^{* * *}}-\underset{(-1.97)}{0.0513^{* *}} \cdot \overline{\log V}_{i}+\epsilon_{i}, \quad i=1, \ldots, 442
$$

with T-statistics in parentheses. Thus, when trading log-volume is below the cross-sectional average $\left(\overline{\log }_{i}<0\right)$, the return following a negative multi-jump is more negative. For positive multi-jumps similar, albeit less strong, effect is evident but in the opposite direction. Fig. 8 delivers the same result with a different technique. We create 40 portfolios sorted on average trading volume. The figure shows the median return after a multi-jump day realized by these portfolios, after good and bad news (their average corresponds to the first points in Panels D and F of Fig. 6). Consistent with estimates of regression models (13) and (14), more illiquid portfolios display stronger predictability, realizing a higher than average positive return after good news and a lower than average negative return after bad news. This evidence supports, again, the idea that low-volume stocks take more time to learn about market-wide news.

\section{Implications for second order moments}

We discuss here the role of multi-jumps for the variance risk premium evolution, for the dynamic of realized variances and for the evolution of asset correlation.

\subsection{Impact on the variance risk premium}

This subsection shows the relevance of detecting multi-jumps by associating their occurrence to changes in the variance risk premium. The variance risk premium on day $t$ for a given maturity $\tau$ is defined as

$$
V R P_{t}=\mathbf{E}^{\mathcal{Q}}[Q V(t, t+\tau)]-\mathbf{E}^{\mathcal{P}}[Q V(t, t+\tau)]
$$




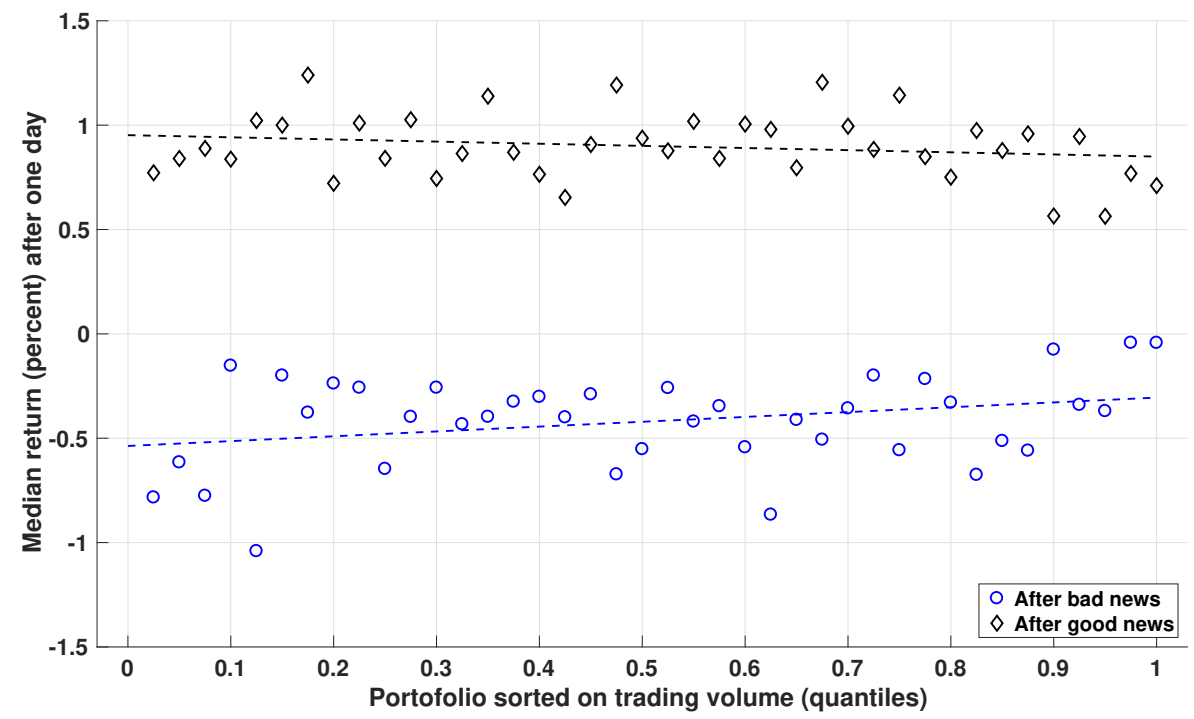

Figure 8: Predictability breakdown by liquidity. We create forty portfolios, sorted on trading volume, and we plot the median return of the portfolios in the day following the occurrence of multi-jumps listed in Table 1 separated between good and bad news. All portfolios have consistently negative median return after bad news and positive median return after good news. However, as also shown by the regression lines, more illiquid portfolios tend to have lower negative returns, and higher positive returns, respectively. This is consistent with the volume effect explained in the text.

where $\mathbf{E}^{\mathcal{Q}}[Q V(t, t+\tau)]$ is the expected risk-neutral quadratic variation of the stock index between times $t$ and $t+\tau$ and $\mathbf{E}^{\mathcal{P}}[Q V(t, t+\tau)]$ is the expected quadratic variation in the same interval. Bollerslev et al. (2008b) highlight the empirical potential of the variance risk premium by showing that $V R P_{t}$ carries significant forecasting power for future returns; see also Carr and Wu (2009) and Bollerslev and Todorov (2011). We use $\tau=1$ month, and we estimate $V R P_{t}$ in our sample using

$$
\widehat{V R P}_{t}=V I X_{t, t+30}^{2}-\widetilde{R V}_{t, t+30}
$$

where $V I X_{t, t+30}$ is the 30-day VIX computed by the Chicago Board Option Exchange that is, the modelfree implied volatility (Jiang and Tian, 2005), and $\widetilde{R V}_{t, t+30}$ is the forecasted realized variance in the same period obtained with the simple regression

$$
\log R V_{t, t+30}=\alpha_{1}+\alpha_{2} \log R V_{t-30, t-1}+\alpha_{3} \log R V_{t-90, t-1}+\varepsilon_{t},
$$

where $\varepsilon_{t}$ is noise and

$$
R V_{t, t+h}=252 \cdot \psi \cdot \sum_{t \leq t^{\prime} \leq t+h} \mathrm{RV}_{t^{\prime}},
$$

with $\mathrm{RV}_{t^{\prime}}$ being the five-minute open-to-close realized variance on day $t$, properly rescaled by 252 (to convert it to yearly units) and by the constant $\psi$, which is the ratio between the sum of squared close-toclose $\mathrm{S} \& \mathrm{P} 500$ daily returns and the average of RV in the sample and which is meant to take into account the contribution of overnight returns to the total variance. Alternative models to forecast variance for the estimation of the variance risk premium, as those considered in Bekaert and Hoerova (2014), yield virtually identical results.

The time series of the estimated variance risk premium in our sample is shown in Fig. 9. As expected, it is almost always positive. We associate it with multi-jumps by using the regression models

$$
\widehat{V R P}_{t}=\gamma_{0}+\gamma_{1} \widehat{V R P}_{t-1}+\gamma_{\mathrm{M}\lrcorner}^{+} \mathrm{MJ}_{t}^{+}+\gamma_{\mathrm{M} J}^{-} \mathrm{MJ}_{t}^{-}+\epsilon_{t},
$$




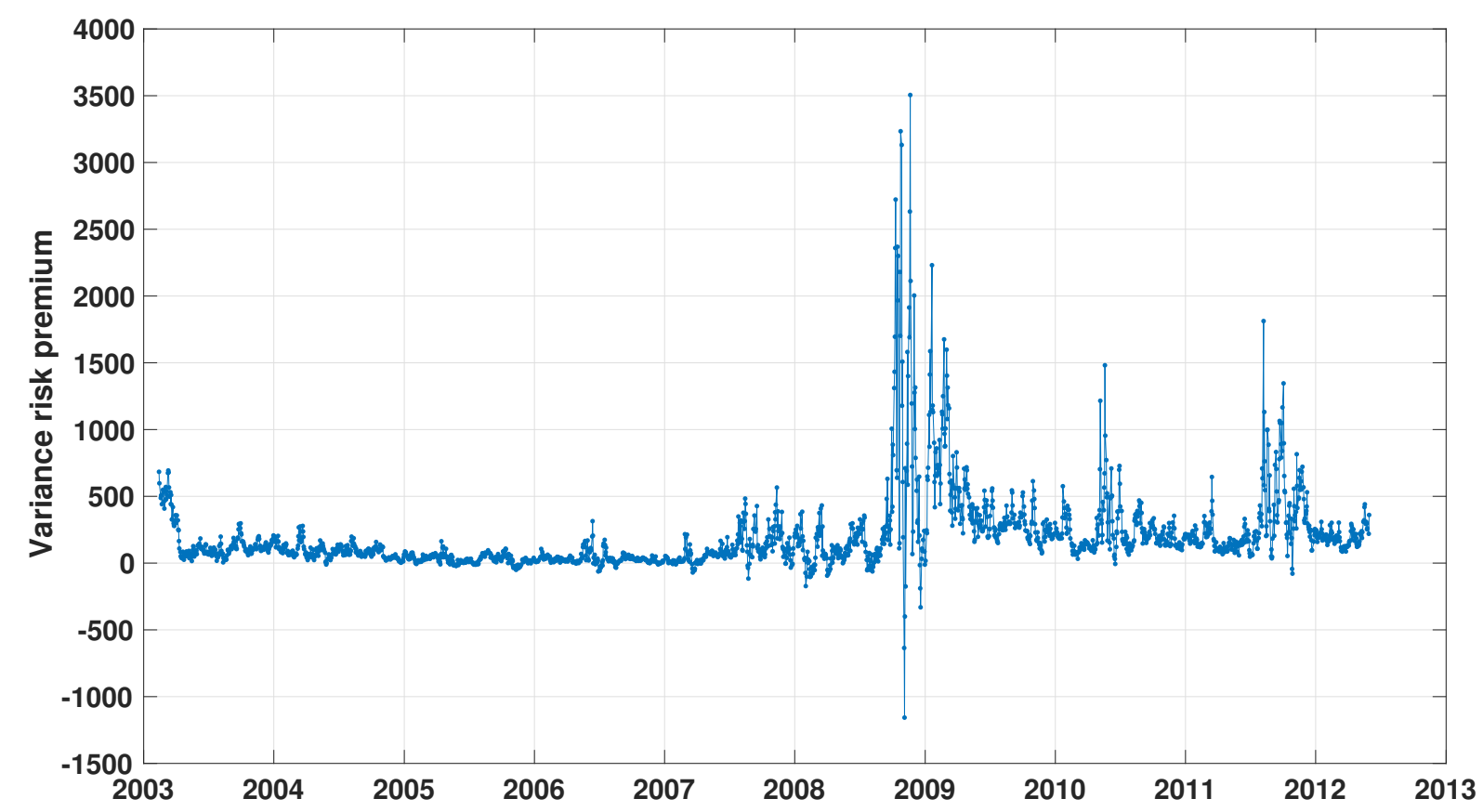

Figure 9: Variance risk premium. The figure reports the daily estimated variance risk premium, computed as in Eq. 16, for the available sample.

where $t$ denotes the day, $\mathrm{MJ}_{t}^{ \pm}$are indicator functions for the presence of multi-jumps associated with bad news (when the contemporaneous return on S\&P 442 is negative) and good news (when the contemporaneous return on $\mathrm{S} \& \mathrm{P} 442$ is positive), and $\epsilon_{t}$ are shocks with zero mean and finite variance. We also run the same regression with lagged dummy variables, that is with $\mathrm{MJ}_{t}^{ \pm}$replaced by $\mathrm{MJ}_{t-1}^{ \pm}$, to examine the predictive power of multi-jumps on the variance risk premium. Estimation results, with Newey-West robust standard errors, are presented in Table 2 for various multi-jump test bandwidth parameters (we use the small, intermediate, and large bandwidths, as defined above).

We find that the constant $\gamma_{0}$ and the autoregressive coefficient $\gamma_{1}$ are strongly significant. More important, our results show that the occurrence of multi-jumps on the variance risk premium is strong and significant, with positive multi-jumps being associated with a decrease of the VRP and negative multi-jumps being associated with an increase (larger, in size) of the VRP. Together with negative multijumps with the large bandwidth, we observe an average increase of the VRP of nearly 140 points, which is also approximately the average variance risk premium in our sample (193.6). This means that variance premium almost doubles (on average) in days with a multi-jump and a downturn of the market. The impact of positive multi-jumps is significant, in the opposite direction (decreasing VRP) and less strong in the same day, however, their effect is significantly negative also the next day, as shown by the lagged regressions. This finding is in keeping with the evidence of Amengual and Xiu (2015), who show that FOMC meetings and Fed speeches indicating an improvement of economic conditions imply a decrease in the variance risk premium.

Systemic co-jumps can be associated with significant changes in the variance risk premium. From a theoretical point of view, our finding corroborates the view that non-Gaussian shocks to fundamentals (sometimes referred to as disasters) have a substantial impact on risk premia; see, e.g. Barro (2006); Gabaix (2012) and Drechsler and Yaron (2011); Drechsler (2013) for economic models directly focusing 
Table 2: Variance risk premium regressions. This table reports estimates (Newey-West standard errors) of model 18 with different restrictions and different choices of the multi-jump dummy. Panel A shows estimates for regressions with contemporaneous multi-jump dummies. Panel B shows estimates for regressions with lagged multi-jump dummies. Tstatistics are in parenthesis, ${ }^{*},{ }^{* *},{ }^{* * *}$ denote significance at the $90 \%, 95 \%$ and $99 \%$ respectively.

\begin{tabular}{|c|c|c|c|c|c|c|c|}
\hline \multicolumn{8}{|c|}{ Panel A: Contemporaneous regressions } \\
\hline$\gamma_{0}$ & $\begin{array}{l}32.1^{* * *} \\
(5.32)\end{array}$ & $\begin{array}{l}30.2^{* * *} \\
(5.15)\end{array}$ & $\begin{array}{l}31^{* * *} \\
(5.21)\end{array}$ & $\begin{array}{l}31.2^{* * *} \\
(5.23)\end{array}$ & $\begin{array}{l}30.7^{* * *} \\
(5.21)\end{array}$ & $\begin{array}{l}31.6^{* * *} \\
(5.26)\end{array}$ & $\begin{array}{l}31.7^{* * *} \\
(5.27)\end{array}$ \\
\hline$\gamma_{1}$ & $\begin{array}{l}0.835^{* * *} \\
(24)\end{array}$ & $\begin{array}{l}0.834^{* * *} \\
(24.6)\end{array}$ & $\begin{array}{l}0.835^{* * *} \\
(24)\end{array}$ & $\begin{array}{l}0.836^{* * *} \\
(24)\end{array}$ & $\begin{array}{l}0.834^{* * *} \\
(24.6)\end{array}$ & $\begin{array}{l}0.835^{* * *} \\
(24)\end{array}$ & $\begin{array}{l}0.835^{* * *} \\
(24)\end{array}$ \\
\hline$\gamma_{M J}^{+}($small h $)$ & & $\begin{array}{l}-96.2^{* * *} \\
(-3.52)\end{array}$ & & & $\begin{array}{l}-93.3^{* * *} \\
(-3.38)\end{array}$ & & \\
\hline$\gamma_{M J}^{-}($small h $)$ & & $\begin{array}{l}169^{* * *} \\
(4.41)\end{array}$ & & & $\begin{array}{l}171^{* * *} \\
(4.38)\end{array}$ & & \\
\hline$\gamma_{M J}^{+}($intermediate $\mathrm{h})$ & & & $\begin{array}{l}-57.2^{* * *} \\
(-3.35)\end{array}$ & & & $\begin{array}{l}-50.4^{* * *} \\
(-3.31)\end{array}$ & \\
\hline$\gamma_{M J}^{-}($intermediate $\mathrm{h})$ & & & $\begin{array}{l}115^{* * *} \\
(2.84)\end{array}$ & & & $\begin{array}{l}114^{* * *} \\
(2.76)\end{array}$ & \\
\hline$\gamma_{M J}^{+}($large h $)$ & & & & $\begin{array}{l}-85.6^{* *} \\
(-2.48)\end{array}$ & & & $\begin{array}{l}-74.2^{* *} \\
(-2.48)\end{array}$ \\
\hline$\gamma_{M J}^{-}($large $\mathrm{h})$ & & & & $\begin{array}{l}138^{* *} \\
(2.13)\end{array}$ & & & $\begin{array}{l}135^{* *} \\
(2.04)\end{array}$ \\
\hline$R^{2}$ & $69.9 \%$ & $70.8 \%$ & $70.1 \%$ & $70.0 \%$ & $70.8 \%$ & $70.1 \%$ & $70.1 \%$ \\
\hline \multicolumn{8}{|c|}{ Panel B: Lagged regressions } \\
\hline$\gamma_{0}$ & $\begin{array}{l}31.9^{* * *} \\
(5.28)\end{array}$ & $\begin{array}{l}31.8^{* * *} \\
(5.34)\end{array}$ & $\begin{array}{l}31.6^{* * *} \\
(5.28)\end{array}$ & $\begin{array}{l}31.8^{* * *} \\
(5.3)\end{array}$ & $\begin{array}{l}32^{* * *} \\
(5.34)\end{array}$ & $\begin{array}{l}31.8^{* * *} \\
(5.28)\end{array}$ & $\begin{array}{l}31.9^{* * *} \\
(5.3)\end{array}$ \\
\hline$\gamma_{1}$ & $\begin{array}{l}0.835^{* * *} \\
(24)\end{array}$ & $\begin{array}{l}0.836^{* * *} \\
(23.9)\end{array}$ & $\begin{array}{l}0.835^{* * *} \\
(24)\end{array}$ & $\begin{array}{l}0.835^{* * *} \\
(24)\end{array}$ & $\begin{array}{l}0.836^{* * *} \\
(23.9)\end{array}$ & $\begin{array}{l}0.835^{* * *} \\
(24)\end{array}$ & $\begin{array}{l}0.835^{* * *} \\
(24)\end{array}$ \\
\hline$\gamma_{M J}^{+}(\mathrm{small} \mathrm{h})$ & & $\begin{array}{l}-7.51 \\
(-0.382)\end{array}$ & & & $\begin{array}{l}-5.83 \\
(-0.292)\end{array}$ & & \\
\hline$\gamma_{M J}^{-}($small h $)$ & & $\begin{array}{l}-4.08 \\
(-0.116)\end{array}$ & & & $\begin{array}{l}-5.15 \\
(-0.142)\end{array}$ & & \\
\hline$\gamma_{M J}^{+}($intermediate $\mathrm{h})$ & & & $\begin{array}{l}-7.24 \\
(-0.38)\end{array}$ & & & $\begin{array}{l}-4.44 \\
(-0.233)\end{array}$ & \\
\hline$\gamma_{M J}^{-}($intermediate $\mathrm{h})$ & & & $\begin{array}{l}21.3 \\
(0.497)\end{array}$ & & & $\begin{array}{l}21 \\
(0.461)\end{array}$ & \\
\hline$\gamma_{M J}^{+}$(large h) & & & & $\begin{array}{l}-46.5^{* * *} \\
(-4.55)\end{array}$ & & & $\begin{array}{l}-42.5^{* * *} \\
(-3.61)\end{array}$ \\
\hline$\gamma_{M J}^{-}($large $\mathrm{h})$ & & & & $\begin{array}{l}15.8 \\
(0.222)\end{array}$ & & & $\begin{array}{l}14.8 \\
(0.204)\end{array}$ \\
\hline$R^{2}$ & $69.9 \%$ & $69.9 \%$ & $69.9 \%$ & $69.9 \%$ & $69.9 \%$ & $69.9 \%$ & \\
\hline
\end{tabular}

on the relation between jumps in fundamentals and the variance risk premium. Moreover, Todorov (2010) shows a strong link between index jump measures and variance risk premium changes through the estimation of a parametric model.

\subsection{Impact on stock variances}

We assess the impact of multi-jumps on future variance by estimating the regression model

$$
\begin{aligned}
\log V_{t}^{(i)}= & \beta_{0}^{(i)}+\beta_{d}^{(i)} \log V_{t-1}^{(i)}+\beta_{w}^{(i)} \log V_{t-5: t-1}^{(i)}+\beta_{m}^{(i)} \log V_{t-21: t-1}^{(i)} \\
& +\beta_{+}^{(i, d)} \mathrm{MJ}_{t-1}^{+}+\beta_{+}^{(i, w)} \mathrm{MJ}_{t-5: t-1}^{+}+\beta_{+}^{(i, m)} \mathrm{MJ}_{t-21: t-1}^{+} \\
& +\beta_{-}^{(i, d)} \mathrm{MJ}_{t-1}^{-}+\beta_{-}^{(i, w)} \mathrm{MJ}_{t-5: t-1}^{-}+\beta_{-}^{(i, m)} \mathrm{MJ}_{t-21: t-1}^{-}+\epsilon_{t}^{(i)}, \quad(i=1, \ldots, 406),
\end{aligned}
$$

where $t$ denotes the day, $V_{t}^{(i)}$ is a measure of the continuous part of the quadratic variation of stock $i$ (we use threshold bipower variation, see Appendix B.3, $\mathrm{MJ}^{ \pm}$are dummies indicating the presence of a 
multi-jump with a sign depending on the return of S\&P 442 in that day,

$$
\log V_{t-h: t-1}^{(i)}=\frac{1}{h} \sum_{t^{\prime}=t-h}^{t-1} \log V_{t^{\prime}}^{(i)},
$$

and

$$
\mathrm{MJ}_{t-h: t-1}^{ \pm}=\sum_{t^{\prime}=t-h}^{t-1} \mathrm{MJ}_{t^{\prime}}^{ \pm}
$$

The explanatory variables $\mathrm{MJ}_{t}^{ \pm}$are the same across all stocks. We use the large bandwidth in this exercise, which is the case when 20 multi-jumps are detected. Results with different bandwidths, not reported here, deliver similar results. The variables $\epsilon_{t}^{(i)}$ are potentially correlated (in the spatial sense; see Appendix C shocks with zero mean and finite variance. We also consider an alternative model in which the $\mathrm{MJ}_{t}^{ \pm}$dummies are replaced by the $\mathrm{J}_{t}^{(i) \pm}$ dummy signaling the detection of an idiosyncratic jump in that day (we use the CPR test for detection at the same confidence interval $\alpha^{*}$ ). Also in this case, the sign of the dummy is taken from the sign of the return on the S\&P 442 index (signaling bad or good news in the whole market).

The model is a natural multivariate generalization of the univariate models adopted in Andersen, Bollerslev and Diebold (2007), Corsi et al. (2010) and Busch et al. (2011), which are all based on the Heterogeneous Auto-Regressive (HAR) model of Corsi (2009). The usual hard-to-beat long-range dependence in volatility delivered by the HAR model is complemented by a systemic component (multijumps), which are both allowed to impact persistently in the variance dynamics through their weekly and monthly components. The systemic component can be associated with news, as shown in the empirical work of Lee (2012), Evans (2011), and Gilder et al. (2014). We restrict attention to the 406 stocks that are observed over the whole sample period. We jointly estimate the parameters of the 406 equations system with a feasible generalized least square (FGLS) estimator (see Appendix C for details). The distribution of the $\beta$ coefficients and of their associated T-statistics is shown in Fig. 10, for the models with multijumps and idiosyncratic jumps. We also report, for both models, the average impact of the occurrence of a multi-jump and an idiosyncratic jump on the variance, as implied by estimated parameters 5

Results show a clear impact of multi-jumps on the future variance. Multi-jumps with good news have a negative impact on future variance, and multi-jumps with bad news have a positive impact. The impact is persistent in both cases. Idiosyncratic jumps have no impact. These findings complement the evidence of Corsi et al. (2010) on the impact of jumps on volatility forecasting. Jumps in individual stocks do not seem to possess forecasting power on future stock volatility, unless they occur in multiple stocks, signaling a systemic event instead of an idiosyncratic event. When this happens, we typically see a jump in the stock index, and this explains why jumps are instead very significant in forecasting the index future variance. The negative impact on future volatility of multi-jumps with good news is also a new feature revealed by our analysis, again consistent with the parametric evidence of Amengual and Xiu (2015) about negative volatility jumps.

\footnotetext{
${ }^{5}$ We compute the average impact of multi-jumps and idiosyncratic jumps as follows. For each asset we set initial values as $\log V_{-22}^{(i)}=\log V_{-21}^{(i)}=\ldots=\log V_{0}^{(i)}=\beta_{0}^{(i)} /\left(1-\beta_{d}^{(i)}-\beta_{w}^{(i)}-\beta_{m}^{(i)}\right)$, that is, to the unconditional variance when neglecting the impact of multi-jumps. We then set $\mathrm{MJ}_{0}=1$ and $\mathrm{MJ}_{t}=0$ when $t \neq 0$, and we compute the dynamics of $\log V_{t}^{(i)}$ using model $[19$. Finally, we average across the 406 assets. The same is done for model [20] in Fig. 11
} 
Panel A: Multi-jumps, daily beta

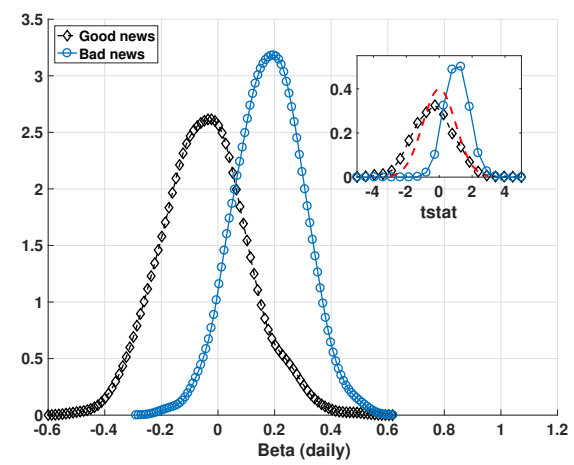

Panel B: Multi-jumps, weekly beta

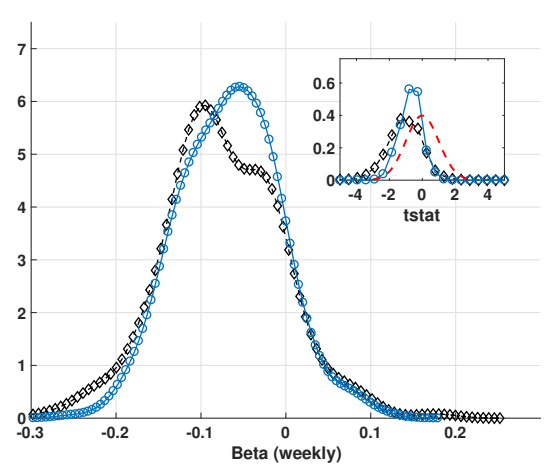

Panel C: Multi-jumps, monthly beta

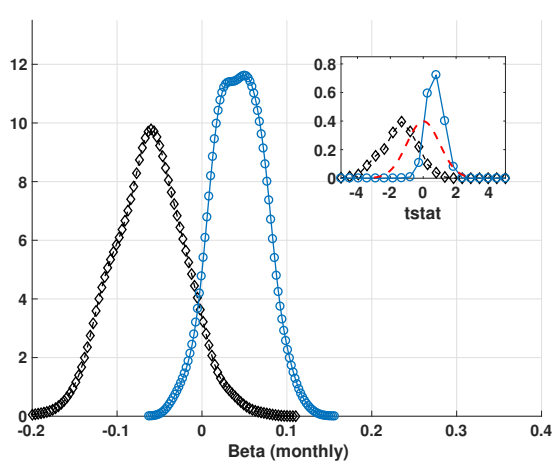

Panel D: Average impact of a systemic multi-jump

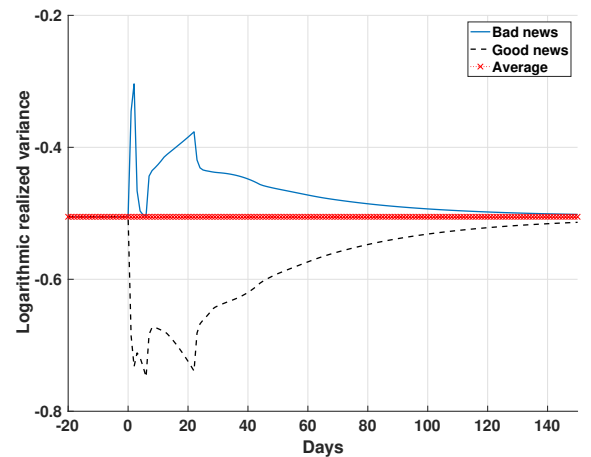

Panel E: Idiosyncratic jumps, daily beta

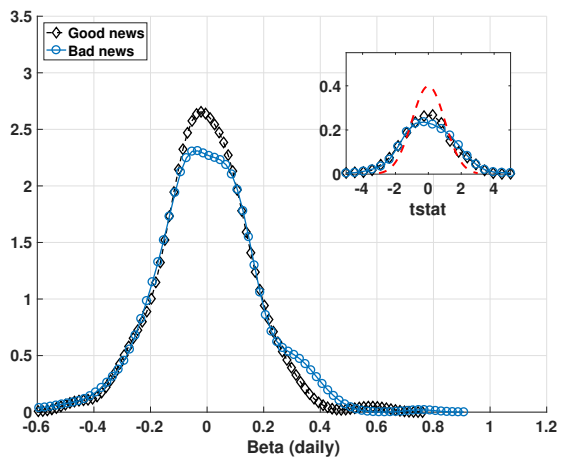

Panel F: Idiosyncratic jumps, weekly beta

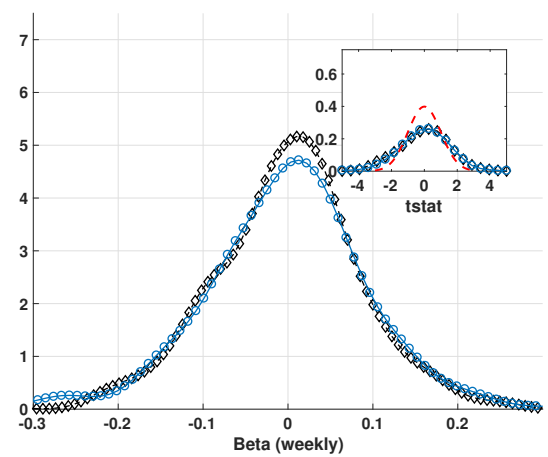

Panel G: Idiosyncratic jumps, monthly beta

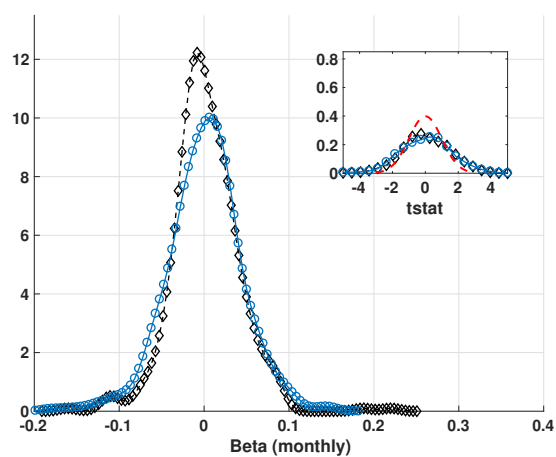

Panel H: Average impact of an idiosyncratic jump

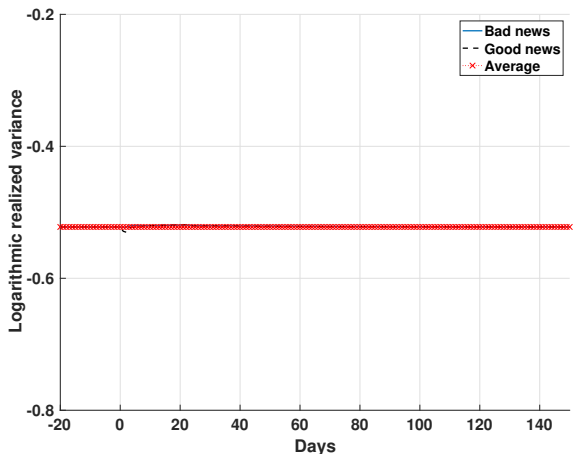

Figure 10: Impact of jumps on volatility. This figure reports the estimated probability density function of the estimated coefficients $\beta_{ \pm}^{(i, d)}, \beta_{ \pm}^{(i, w)}$, and $\beta_{ \pm}^{(i, m)}$ for model $\sqrt{19}$, and the average dynamic impact of the occurrence of a jump (see footnote 5 for details on its computation). Panels A, B, C, D use the multi-jumps dummy. Panels E, F, G, H use individual jump dummies. The insets in panels A, B, C, E, F, G plot the frequency histogram of the associated T-statistics compared with a standard normal distribution (dashed red). 


\subsection{Impact on stock correlations}

We assess the impact of multi-jumps on future stock correlations by estimating the regression model

$$
\begin{aligned}
\widetilde{\rho}_{t}^{(i, j)}= & \beta_{0}^{(i, j)}+\beta_{d}^{(i, j)} \widetilde{\rho}_{t-1}^{(i, j)}+\beta_{w}^{(i, j)} \widetilde{\rho}_{t-5: t-1}^{(i, j)}+\beta_{m}^{(i, j)} \widetilde{\rho}_{t-22: t-1}^{(i, j)} \\
+ & \beta_{+}^{(i, j, d)} \mathrm{MJ}_{t-1}^{+}+\beta_{+}^{(i, j, w)} \mathrm{MJ}_{t-5: t-1}^{+}+\beta_{+}^{(i, j, m)} \mathrm{MJ}_{t-21: t-1}^{+} \\
+ & \beta_{-}^{(i, j, d)} \mathrm{MJ}_{t-1}^{-}+\beta_{-}^{(i, j, w)} \mathrm{MJ}_{t-5: t-1}^{-}+\beta_{-}^{(i, j, m)} \mathrm{MJ}_{t-21: t-1}^{-}+\epsilon_{t}^{(i)}, \\
& (i=1, \ldots, 19, j=i+1, \ldots, 19),
\end{aligned}
$$

estimated on the 19 stocks with larger volume in the sample 6 The model parallels model (19), with the same definitions, but is now specified for Fisher transformation of realized correlations between stock $i$ and stock $j \neq i$ (Appendix B describes how correlations are measured by means of intraday data),

$$
\widetilde{\rho}_{t-h: t-1}^{(i)}=\frac{1}{h} \sum_{t^{\prime}=t-h}^{t-1} \widetilde{\rho}_{t^{\prime}}^{(i)},
$$

and the dummy variables are aggregated as for model $(19)$. We also estimate the model after replacing $\mathrm{MJ}_{t}^{ \pm}$with $\mathrm{CJ}_{t}^{(i, j) \pm}$, an idiosyncratic dummy variable signaling the detection of a multi-jump in the specific pair (we use our multi-jump test with $N=2$ and $h_{n}=6.5$ ). Again, $\epsilon_{t}^{(i, j)}$ are potentially correlated (in the spatial sense) shocks with zero mean and finite variance. As before, we estimate the model for 171 pairs using an FGLS estimator (see Appendix C). Fig. 11 reports the distribution of estimated coefficients, their associated T-statistics, and the average impact of a multi-jump and an idiosyncratic multi-jump (see footnote 5 for details on its computation).

The figure shows that detected multi-jumps have a very strong impact on future covariances with the sign, again, depending on whether they come with bad news or good news. Bad news implies a strong positive impact on the average correlations, and good news implies a negative impact, somewhat less strong. The impact on the average correlation is still persistent, even if not as persistent as for the variance. Idiosyncratic multi-jumps display no forecasting power for correlations. Multi-jumps can again be viewed, by the light of results in Longin and Solnik (2001), who report an increase in market correlations in turmoil periods, as the statistical counterpart of systemic economic events. We complement their evidence by showing that positive multi-jumps decrease stock correlations (and also stock variances and the variance risk premium), so that their arrival determines a normalization of market conditions.

\section{Simulations}

To simulate the dynamics of realistic prices, we generate sample paths of a multivariate model that accommodates correlated prices, stochastic volatility, correlated volatilities, leverage effect, intraday effects, idiosyncratic jumps in prices, jumps in volatilities, and multi-jumps. All these components have been shown to be present in the dynamics of high-frequency financial prices. The dynamics of each component are specified as

$$
d X_{t}^{(i)}=\mu d t+\gamma_{t} \sigma_{t}^{(i)}\left(\rho d W^{\sigma}+\sqrt{1-\rho^{2}} d W_{t}^{(i)}\right)+d J_{t}^{(i)},
$$

\footnotetext{
${ }^{6}$ The 20th largest volume stock is not traded over the whole sample, which is why we exclude it.
} 
Panel A: Multi-jumps, daily beta

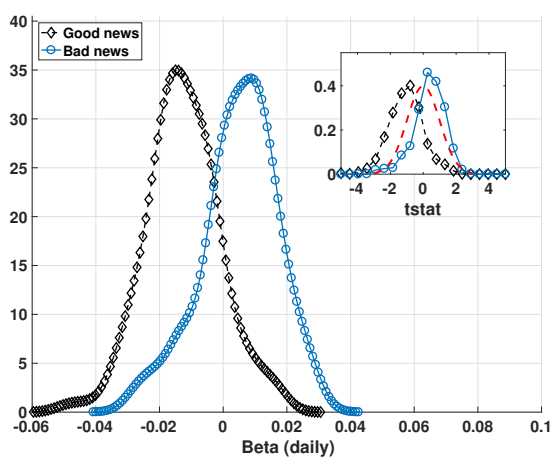

Panel B: Multi-jumps, weekly beta

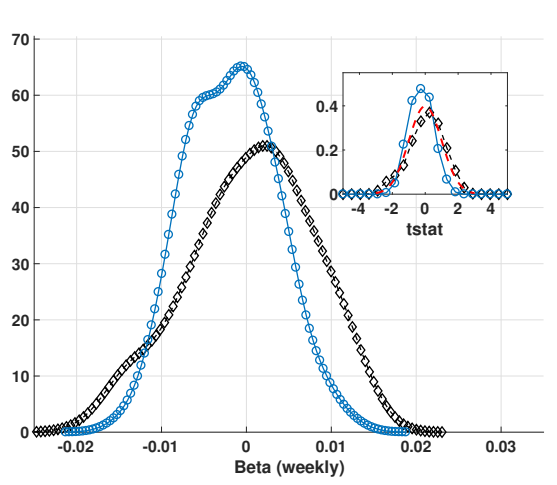

Panel C: Multi-jumps, monthly beta

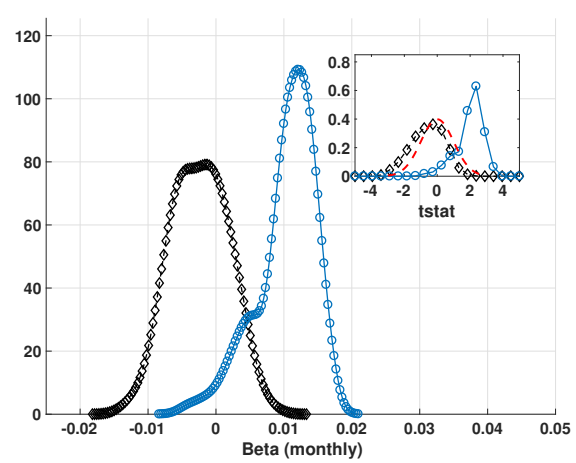

Panel D: Average impact of a systemic multi-jump

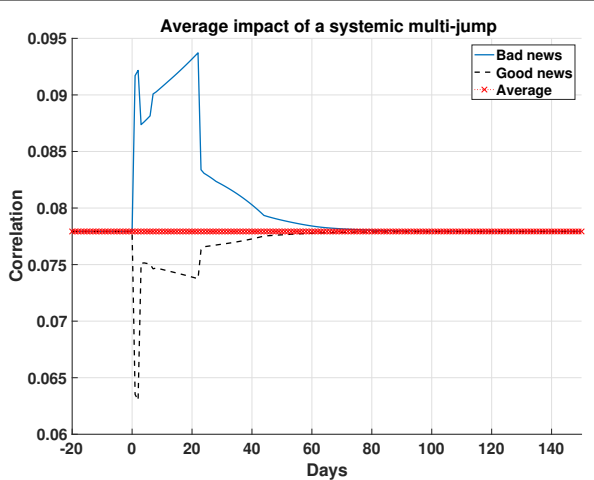

Panel E: Idiosyncratic co-jumps, daily beta

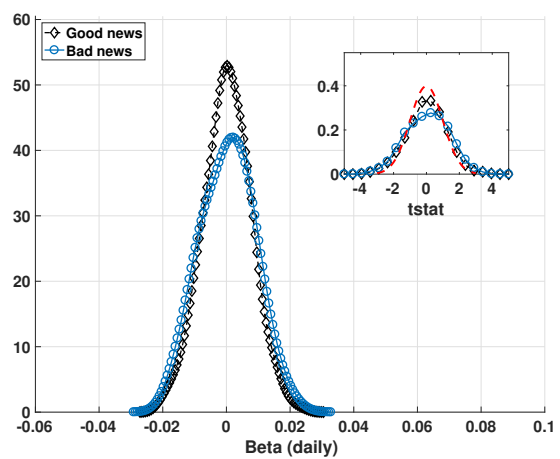

Panel F: Idiosyncratic co-jumps, weekly beta

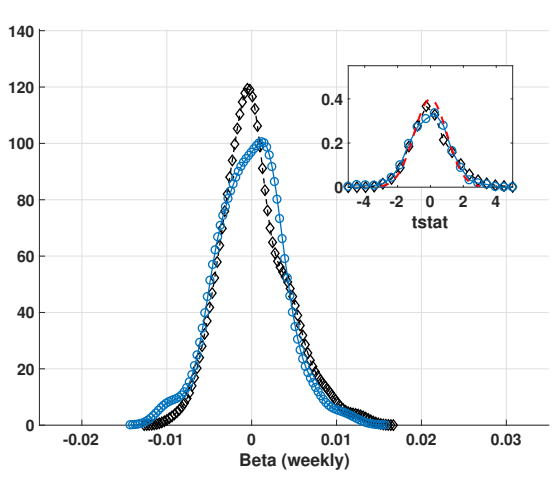

Panel G: Idiosyncratic co-jumps, monthly beta

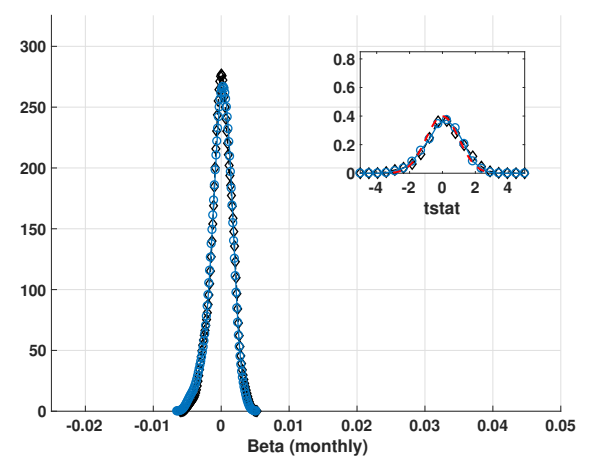

Panel H: Average impact of an idiosyncratic co-jump

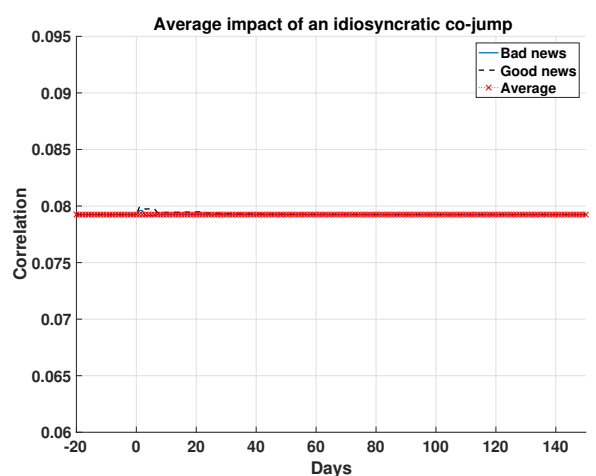

Figure 11: Impact of jumps on correlations. This figure reports the estimated probability density function of the estimated coefficients $\beta_{ \pm}^{(i, j, d)}, \beta_{ \pm}^{(i, j, w)}$, and $\beta_{ \pm}^{(i, j . m)}$ for model 11 , and the average dynamic impact of the occurrence of a jump (see footnote 5 for details on its computation). Panels A, B, C, D use the multi-jumps dummy. Panels E, F, G, H use the dummy for co-jumps between stocks pairs. The insets in panels A, B, C, E, F, G plot the frequency histogram of the associated T-statistics compared with a standard normal distribution (dashed red). 


$$
d \log \left(\sigma_{t}^{(i)}\right)^{2}=\left(\alpha-\beta \log \left(\sigma_{t}^{(i)}\right)^{2}\right) d t+\eta d W^{\sigma}+\eta^{\prime} d \widetilde{W}_{t}^{(i)}+d J_{t}^{\sigma(i)}
$$

where the Brownian motions $W^{(i)}, i=1, \ldots, N$, can be correlated and the Brownian motions $W^{\sigma}$ and $\widetilde{W}^{(i)}, i=1, \ldots, N$, are independent from the $W^{(i)}$ and among them. $\sigma_{t}^{(i)}$ are stochastic volatility factors, and $\gamma_{t}$ represents intraday effects. The Brownian motions $W^{(i)}$ driving the price dynamics can be correlated. The pure jump parts of $X^{(i)}$ are different compound Poisson processes. The volatility in each stock is driven by two factors. The first $\left(W^{\sigma}\right)$ is systemic and affects all volatilities in the same way. The second $\left(\widetilde{W}_{t}^{(i)}\right)$ is idiosyncratic.

The parameters of the model are chosen to guarantee a realistic dynamics: $\mu=0.0304, \alpha=$ $-0.012, \beta=0.0145, \eta=0.1153, \rho=-0.5$ and $\eta^{\prime}=0.3 \eta$. These parameters roughly correspond to the estimates of Andersen et al. 2002) on S\&P 500 returns. All parameters are expressed in daily units and returns are in percentage. The intraday effects are given by

$$
\gamma_{t}=\frac{1}{0.1033}\left(0.1271 \cdot t^{2}-0.1260 \cdot t+0.1239\right)
$$

as estimated by us on S\&P 500 intraday returns. In our simulations, we always have $t \in[0,1]$, with initial values for prices and volatility taken from the last simulated day.

Eqs. (21) and 22 are discretized with the Euler scheme, using discretization step of $\Delta=\frac{1}{80}$, which roughly corresponds to five-minute returns for a trading day of 6.5 hours $(n=80)$. We generate samples of one thousand days with different specifications for the jump processes in price $\left(d J_{t}^{(i)}\right)$ and in volatility $\left(d J_{t}^{\sigma(i)}\right)$. We concentrate here on two relevant cases. Additional simulations can be found in the Online Appendix.

\subsection{Comparison with univariate tests}

We compare the multi-jump test with three univariate jump tests: the CPR test of Corsi et al. (2010), the BNS test of Barndorff-Nielsen and Shephard (2006) and the ABD test of Andersen, Bollerslev and Dobrev (2007), all described in Appendix B.2. We apply univariate tests on an equally weighted $(1 / N)$ portfolio of simulated stocks. We simulate one thausand paths of $N=16$ stocks. Continuous parts of all components follow Eqs. (21) and (22). The Brownian motions driving the system are correlated with the average daily correlation matrix estimated on actual stock returns (we use 16 stocks from four different economic sectors). We consider three cases. In Case 1, each path contains a single multi-jump across the 16 stocks, with jump sizes being normally distributed, the mean being equal to $8 \sqrt{\Delta}$, and the standard deviation being equal to $2 \sqrt{\Delta}$. In Case 2 , the multi-jump in price is accompanied by a substantial increase in the volatility of volatility, obtained by multiplying the parameter $\eta$ by 10 . In Case 3 , the multi-jump in price is accompanied by a contemporaneous multi-jump in the volatility of each stock. The simulated jump sizes are sufficiently large on average but show dispersion such that some of the univariate jumps could be smaller (the continuous daily variance hovers around $1 \%$ in daily units).

Table 3 shows power for the multi-jump tests based on the $S_{n, N}$ statistics and the three univariate tests in the portfolio. When the volatility of volatility is low, the $S_{n, N}$ proposed here and the univariate tests in the portfolio are equally powerful. When the price multi-jump is accompanied by an increase of the volatility of volatility, the test in the portfolio loses power significantly, and the multi-jump test is practically not affected. The loss in power is particularly severe for the BNS test, and it is not so strong for the ABD test, which, however, has a large size distortion in the absence of jumps (see Table B.4 in the Appendix). 
Table 3: Power of jump tests on simulated data. This table shows the power of the proposed multi-jump test in comparison with the univariate jump test on an equally weighted portfolio. Different processes for the alternative are simulated, as described in the text. The sampling frequency $n=80$ corresponds to five-minute intraday observations. CPR $=$ Corsi, Pirino and Renò; BNS=Barndorff-Nielsen and Shephard; ABD=Andersen, Bollerslev and Dobrev.

\begin{tabular}{lllll}
\multicolumn{6}{c}{ Confidence interval } \\
\hline $90 \%$ & $95 \%$ & $99 \%$ & $99.9 \%$ & $99.995 \%$ \\
\hline
\end{tabular}

Case 1: Low volatility of volatility

Multi-jump test

$\begin{array}{llllll}\mathrm{S}_{80,16}(2) & 99.94 & 99.94 & 99.93 & 99.92 & 99.91\end{array}$

Univariate tests on the $1 / N$ portfolio

$\begin{array}{lccccc}\text { CPR } & 100.00 & 100.00 & 100.00 & 100.00 & 100.00 \\ \text { BNS } & 100.00 & 100.00 & 99.95 & 99.67 & 97.48 \\ \text { ABD } & 100.00 & 100.00 & 100.00 & 100.00 & 100.00\end{array}$

Case 2: High volatility of volatility

Multi-jump test

$\begin{array}{llllll}\mathrm{S}_{80,16}(2) & 58.70 & 56.14 & 53.43 & 52.21 & 51.42\end{array}$

Univariate tests on the $1 / N$ portfolio

$\begin{array}{lccccc}\text { CPR } & 22.31 & 16.23 & 8.31 & 4.57 & 2.68 \\ \text { BNS } & 18.51 & 12.43 & 5.99 & 2.81 & 1.63 \\ \text { ABD } & 57.33 & 47.12 & 31.26 & 19.36 & 11.83\end{array}$

For the relevant case in which the multi-jump in the simulated stocks is accompanied by a multi-jump in the volatilities (with the same size across all stocks), we show in Fig. 12 the power of the multi-jump test with different bandwidths $\left(h_{n}=1,1.5,2,4.5\right)$ as a function of the volatility jump size (expressed in yearly units) 7 . The jump in volatility (as for the increase in the volatility of volatility parameter) does not affect the power of the multi-jump test. Fig. 12 also shows the harmful effect of the volatility jump size on the univariate test on the portfolio. The multi-jump test appears overall to have a better signal-to-noise ratio than competitors, and this result is consistent with data analysis.

\subsection{Simulating large panels}

In this subsection, we simulate the impact of the trading mechanism on the price discovery using large panels, up to $N=400$. To simulate a realistic environment for a multi-jump day, we multiply the volatility of volatility parameter $\eta$ by a factor of five. When we compute power, we add to the simulated price a single multi-jump of size $8 \sqrt{1 / 80}$ to each simulated stock. The Brownian motions driving the system are correlated with constant correlation equal to 0.3. We simulate one thousand paths of oneminute $(n=400)$ prices. The multi-jump is located randomly within the five-minute interval.

7 The fact that jumps in prices are typically accompanied by jumps in volatility has been empirically shown by Todorov and Tauchen (2011) and Bandi and Renò (2016), among others. 


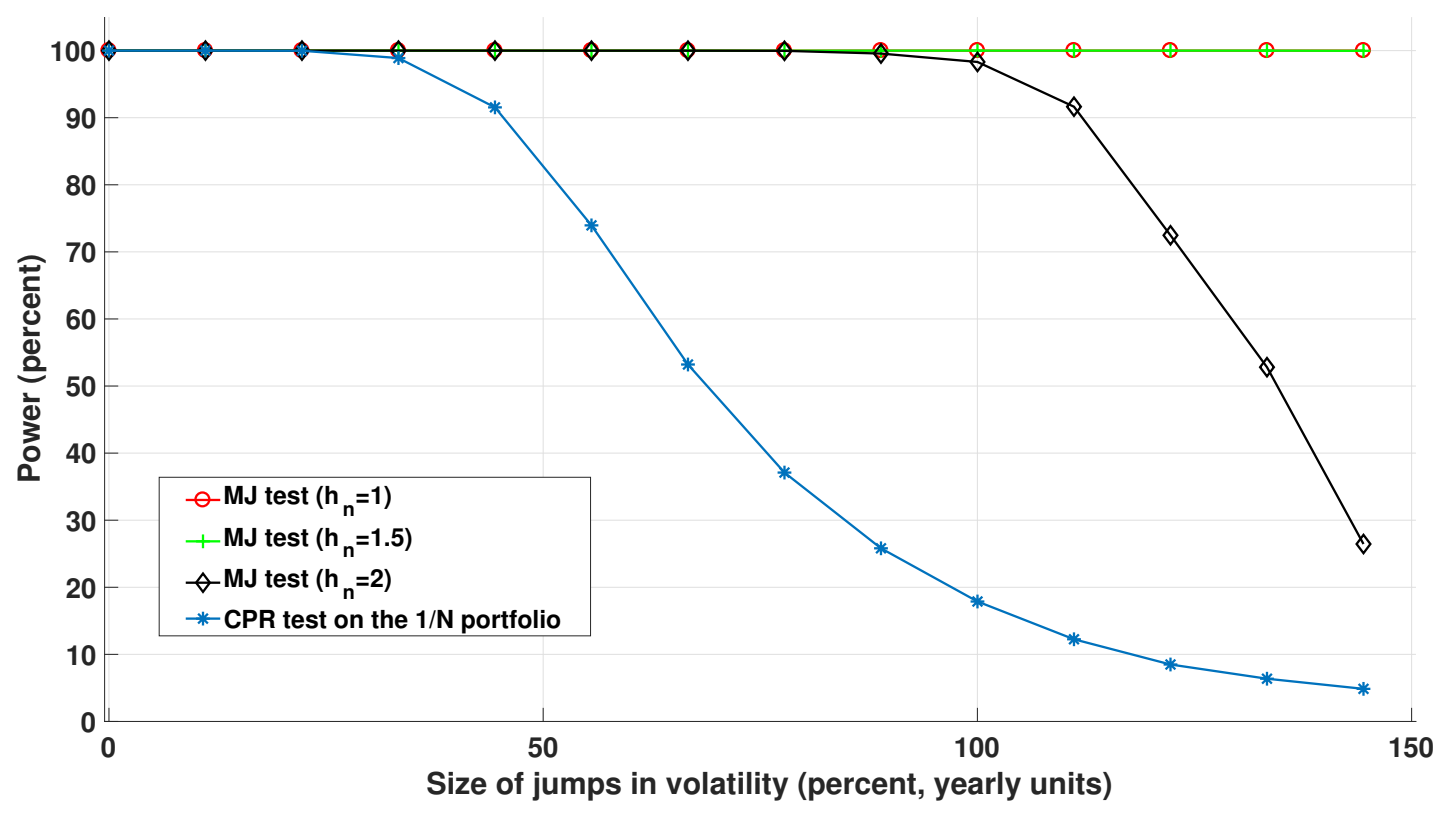

Figure 12: The impact of volatility jumps. This figure shows the power of the multi-jump test with three different bandwidths and the CPR test on the $1 / N$ portfolio on simulated data in which a multi-jump in stocks is accompanied by a multi-jump in the volatility of each stock, as a function of the jump size expressed in percentage and yearly units.

We also simulate the frictional model proposed by Hasbrouck and Ho (1987) and Amihud and Mendelson (1987), to mimic differential incorporation of news in the traded price. We assume that Eqs. 21) and 22 hold for the fundamental price $X_{t}^{(i)}$. The traded log-price $p_{t}^{(i)}$ of the ith security, observed discretely at time $t_{l}=k \tau$, for $k=0,1, \ldots$, instead follows

$$
p_{t_{k}}^{(i)}=p_{t_{k-1}}^{(i)}+\delta^{(i)} \cdot\left(X_{t_{k}}^{(i)}-p_{t_{k-1}}^{(i)}\right), \quad i=1, \ldots, N .
$$

The dynamics models partial adjustment of the traded price to the fundamental price. The parameters $\delta^{(i)}$ reflect the speed of adjustment. A value of $\delta=1$ represents the frictionless case in which the traded price coincides with the fundamental price. Values of $\delta<1$ represent partial price adjustment. For each generation, we sample the parameters $\delta^{(i)}$ from a uniform distribution in the interval $[0.2,1]$, to simulate fast learning stocks, for 50 stocks, and from a uniform distribution in the interval $[0,0.2]$, to simulate slow learning stocks, for the remaining 350 stocks.

We apply the proposed multi-jump test to the five-minute returns constructed from the aggregation of simulated one-minute prices of $N \leq 400$ stocks ranked with respect to the values of their $\delta \mathrm{s}$, so that stocks that learn faster are used first or to a random selection of stocks. The bandwidth and the confidence level are chosen exactly as in the empirical application in Section 4 (we use the large bandwidth here).

Fig. 13 displays the power and size of the tests as a function of $N$. Results when sorting with the learning parameters are displayed with a solid line, and those when selecting the stocks randomly are displayed with a dotted line. Panels $\mathrm{A}$ and $\mathrm{C}$ show the results without partial adjustment effects, and Panels D and F show the outcome with partial adjustment. Hump-shape is evident in the power of the multi-jump test, which is consistent with that observed on real data. The CPR and BNS tests look more stable with respect to $N$, even if less powerful. When frictional partial adjustment is added, the power of the multi-jump test is compromised, unless we select the stocks using those with high learning parameter first. In this case, no power loss is observed at all. This result is important as it is in line with that 
$\underline{\text { Panel A: Power, immediate learning }}$

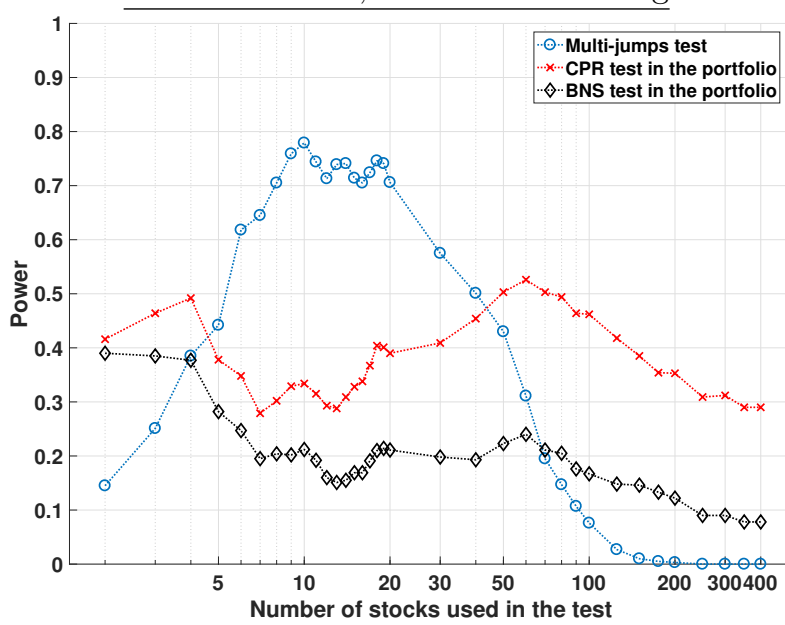

Panel B: Size at 5\%, immediate learning

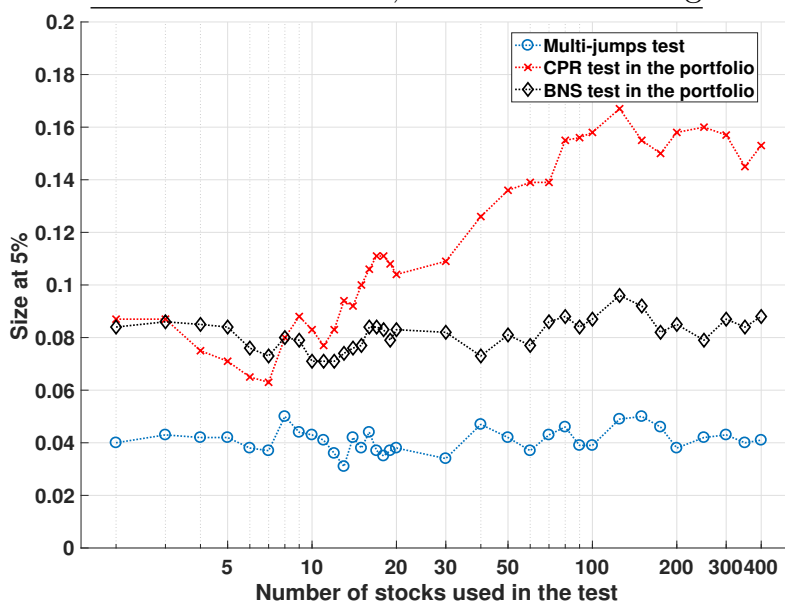

Panel C: Size at $\alpha^{*}=4.8 \cdot 10^{-5}$, immediate learning

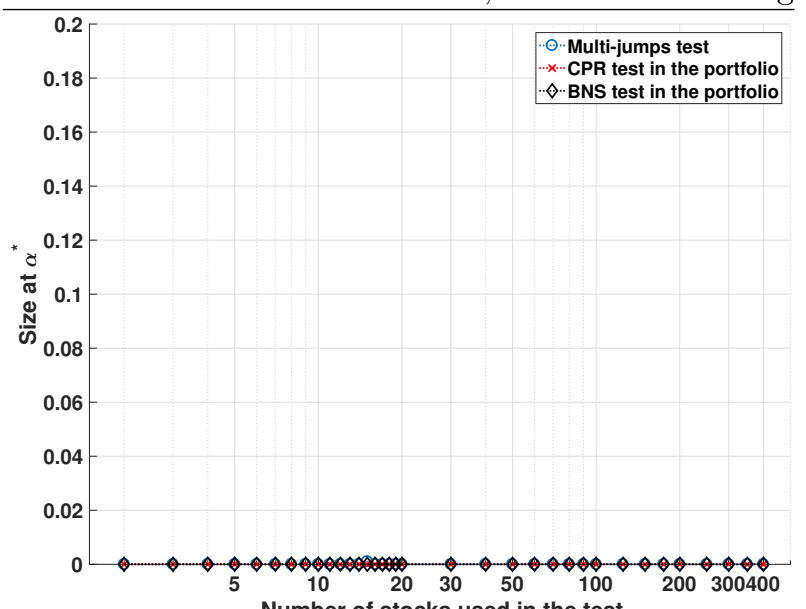

$\underline{\text { Panel D: Power, partial learning }}$

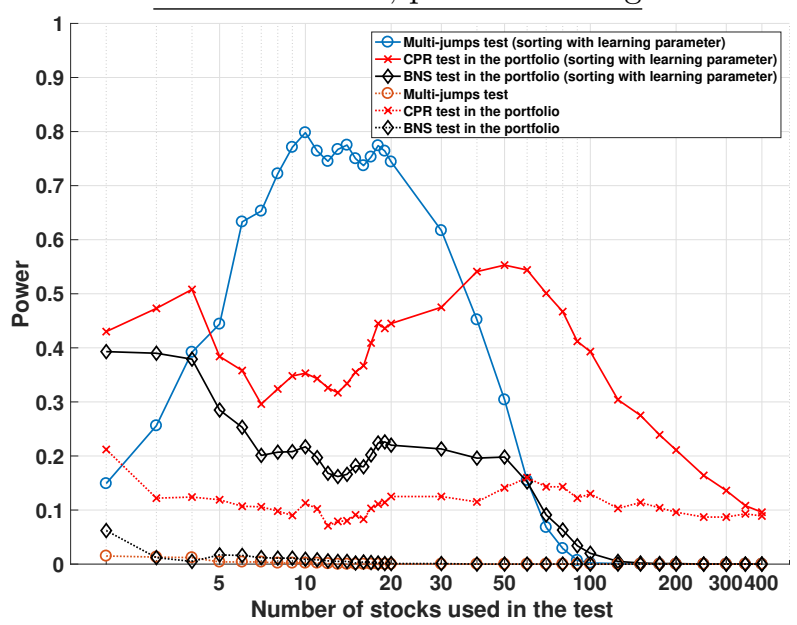

Panel E: Size at 5\%, partial learning

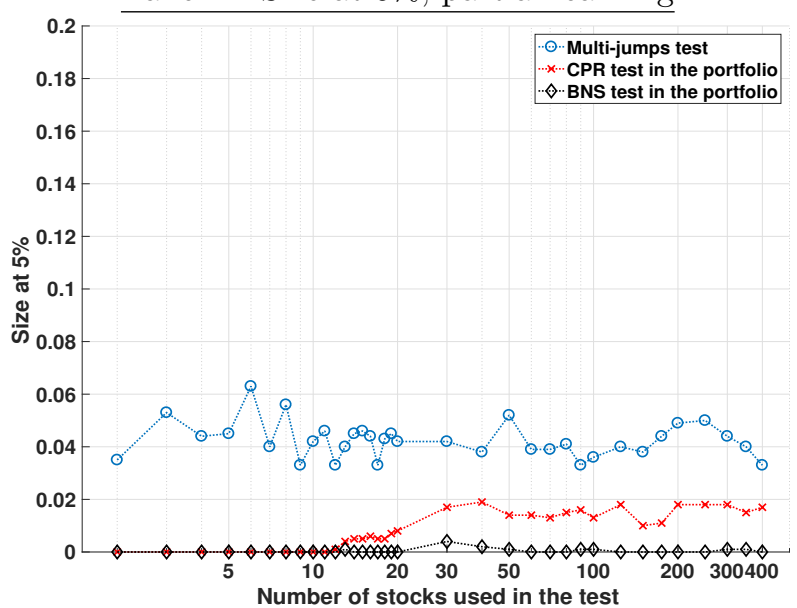

Panel F: Size at $\alpha^{*}=4.8 \cdot 10^{-5}$, partial learning

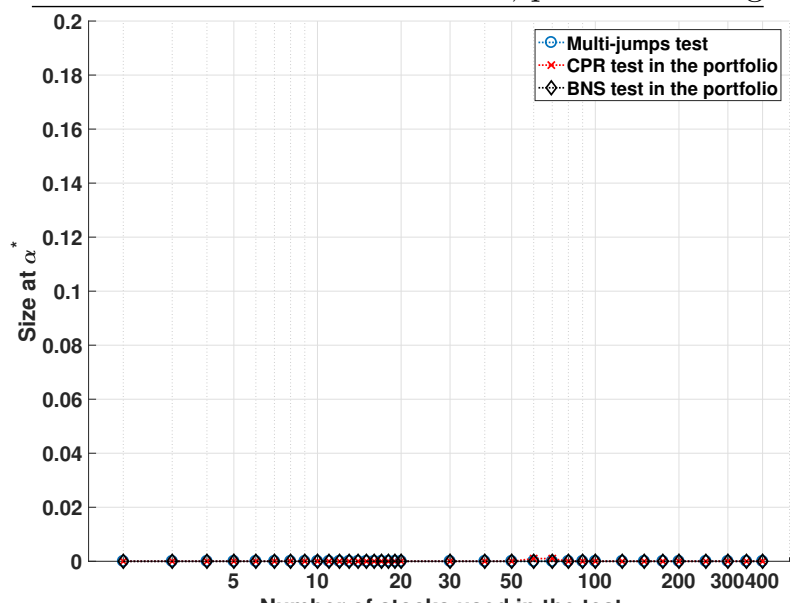

Number of stocks used in the test

Figure 13: The impact of partial learning. The figure shows the power and size (at $5 \%$ and $\alpha^{*}$ confidence levels) of the multi-jump on $N$ stocks and of the CPR and BNS tests on the portfolio of $N$ simulated stocks, as a function of $N$. Panels $\mathrm{A}, \mathrm{B}$ and $\mathrm{C}$ show the result in the case with no partial adjustment. Panels D, E and F show the case in which partial adjustment effects are added to the baseline model. Solid lines are for the case in which the $N$ stocks are selected according to the highest learning parameter; dashed lines, for the case in which the stocks are selected randomly. 
in Fig. 2, in which instead of ranking with respect to the learning parameter $\delta$, we rank with respect to trading volume, interpreting volume as a measure of speed of learning. BNS test is also strongly affected by partial adjustment, and the CPR test appear more robust to this kind of friction but still considerably less powerful in its presence. Thus, in addition to volatility spikes, partial adjustment can affect negatively univariate jump test statistics.

The multi-jump test is well sized at $5 \%$ and at $\alpha^{*}$, where we expect no signal on our one thousand replications, and the size of the test is robust to partial adjustment effects. The CPR and BNS tests suffer severe size distortion at $5 \%$, being oversized in the case without partial adjustment and undersized in the case with partial adjustment.

These results on simulated data confirm our intuition on the effect of learning on the power of the multi-jump test provided in Section 4 that is low $\delta$ stocks incorporate information slowly and, thus, make the detection of the jump less powerful. To maximize power, it is better to choose an intermediate number of stocks and those that learn faster (in the empirical application, our proxy of fast learning being high traded volume).

\section{Concluding remarks}

While the recent literature offers abundant theoretical contributions about the presence of jumps in financial prices and their importance in financial models, much less effort has been devoted to simultaneous co-jumps in a collection of stocks (multi-jumps). Applying a novel test to individual high-volume stocks on a data set including a large fraction of the constituents of the S\&P 500 index, we provide clear-cut evidence on the presence of rare systemic co-jumps in the market, and we show that they can be used to predict stock returns in the short term. We also exhibit their correlation with changes in the variance risk premium. Furthermore, we find an increase (decrease) in both stock volatilities and stock correlations in days following the occurrence of multi-jumps, associated with bad (good) news, with a persistent impact that contrasts the typical transient impact associated with jumps in the index.

Building on empirical evidence (either on real data and simulations), we conclude that testing for multi-jumps with high-volume stocks is much more informative than testing for univariate jumps in portfolios, either stock indices or high-volume portfolios, so that our technique should replace traditional tests when looking for systemic events that affect market variables such as variances, correlations, and risk premia. The tool introduced in this paper could then become of fundamental help in assessing the role, both theoretical and empirical, of jumps in financial markets.

Our work also lays down some new probative research questions. Our empirical findings are consistent with the idea, grounded on microstructural models of price formation, that stocks incorporate information heterogeneously. We identify the ability of incorporating information with trading volume. The full assessment of the relation between trading volume and the speed of reaction to jumps in fundamental values requires the specification of an alternative structural model at frequencies that are higher than those used in this paper. We leave this work for future research. 


\section{References}

Aït-Sahalia, Y., Jacod, J., 2009. Testing for jumps in a discretely observed process. Annals of Statistics $37,184-222$.

Ait-Sahalia, Y., Jacod, J., 2012. Analyzing the spectrum of asset returns: Jump and volatility components in high frequency data. Journal of Economic Literature 50 (4), 1007-1050.

Amengual, D., Xiu, D., 2015. Resolution of policy uncertainty and sudden declines in volatility, working Paper.

Amihud, Y., 2002. Illiquidity and stock returns: cross-section and time-series effects. Journal of Financial Markets 5 (1), 31-56.

Amihud, Y., Mendelson, H., 1987. Trading mechanism and stock retuns: An empirical investigation. The Journal of Finance 42 (3), 533-553.

Andersen, T., Benzoni, L., Lund, J., 2002. An empirical investigation of continuous-time equity return models. Journal of Finance 57, 1239-1284.

Andersen, T., Bollerslev, T., Diebold, F. X., 2007a. Roughing it up: Including jump components in the measurement, modeling and forecasting of return volatility. Review of Economics and Statistics 89, 701-720.

Andersen, T., Bollerslev, T., Dobrev, D., 2007b. No-arbitrage semi-martingale restrictions for continuoustime volatility models subject to leverage effects, jumps and iid noise: Theory and testable distributional implications. Journal of Econometrics 138 (1), 125-180.

Back, K., 1991. Asset pricing for general processes. Journal of Mathematical Economics 20 (4), 371-395.

Bajgrowicz, P., Scaillet, O., Treccani, A., 2016. Jumps in high-frequency data: Spurious detections, dynamics, and news. Management Science 62 (8), 2198-2217.

Bandi, F., Renò, R., 2016. Price and volatility co-jumps. Journal of Financial Economics 119 (1), $107-146$.

Barndorff-Nielsen, O., Shephard, N., 2003. Measuring the impact of jumps in multivariate price processes using bi-power covariation, working paper, Nuffield College, Oxford University.

Barndorff-Nielsen, O. E., Shephard, N., 2006. Econometrics of testing for jumps in financial economics using bipower variation. Journal of Financial Econometrics 4, 1-30.

Barro, R., 2006. Rare disasters and asset markets in the twentieth century. The Quarterly Journal of Economics 121 (3), 823-866.

Bekaert, G., Hoerova, M., 2014. The VIX, the variance premium and stock market volatility. Journal of Econometrics 183 (2), 181-192.

Bibinger, M., Winkelmann, L., 2015. Econometrics of co-jumps in high-frequency data with noise. Journal of Econometrics 184 (2), 361-378.

Billio, M., Getmansky, M., Lo, A. W., Pelizzon, L., 2012. Econometric measures of connectedness and systemic risk in the finance and insurance sectors. Journal of Financial Economics 104 (3), 535-559.

Bollerslev, T., Law, T. H., Tauchen, G., 2008a. Risk, jumps and diversification. Journal of Econometrics 144, 234-256.

Bollerslev, T., Tauchen, G., Zhou, H., 2008b. Expected stock returns and variance risk premia. Review of Financial Studies 22 (11), 4463-4492.

Bollerslev, T., Todorov, V., 2011. Tails, fears, and risk premia. The Journal of Finance 66 (6), 2165-2211.

Busch, T., Christensen, B., Nielsen, M., 2011. The role of implied volatility in forecasting future realized volatility and jumps in foreign exchange, stock, and bond markets. Journal of Econometrics 160, 48-57.

Carr, P., Wu, L., 2009. Variance risk premiums. Review of Financial Studies 22 (3), 1311-1341.

Christensen, K., Oomen, R. C., Podolskij, M., 2014. Fact or friction: Jumps at ultra high frequency. Journal of Financial Economics 114 (3), 576-599. 
Corsi, F., 2009. A simple approximate long-memory model of realized volatility. Journal of Financial Econometrics 7, 174-196.

Corsi, F., Pirino, D., Renò, R., 2010. Threshold bipower variation and the impact of jumps on volatility forecasting. Journal of Econometrics 159, 276-288.

Das, S., Uppal, R., 2004. Systemic Risk and International Portfolio Choice. The Journal of Finance 59 (6), 2809-2834.

Drechsler, I., 2013. Uncertainty, time-varying fear, and asset prices. The Journal of Finance 68 (5), 1843-1889.

Drechsler, I., Yaron, A., 2011. What's vol got to do with it. Review of Financial Studies 24 (1), 1-45.

Dumitru, A.-M., Urga, G., 2012. Identifying jumps in financial assets: a comparison between nonparametric jump tests. Journal of Business \& Economic Statistics 30 (2), 242-255.

Evans, K. P., 2011. Intraday jumps and us macroeconomic news announcements. Journal of Banking \& Finance 35 (10), 2511-2527.

Gabaix, X., 2012. Variable rare disasters: An exactly solved framework for ten puzzles in macro-finance. The Quarterly Journal of Economics 127 (2), 645-700.

Gilder, D., Shackleton, M. B., Taylor, S. J., 2014. Cojumps in stock prices: Empirical evidence. Journal of Banking \& Finance 40, 443-459.

Hasbrouck, J., Ho, T., 1987. Order arrival, quote behavior, and the return-generating process. The Journal of Finance 42 (4), 1035-1048.

Hautsch, N., Podolskij, M., 2013. Preaveraging-based estimation of quadratic variation in the presence of noise and jumps: theory, implementation, and empirical evidence. Journal of Business \& Economic Statistics 31 (2), 165-183.

Jacod, J., Todorov, V., 2009. Testing for common arrivals of jumps for discretely observed multidimensional processes. Annals of Statistics 37, 1792-1838.

Jiang, G., Oomen, R., 2008. Testing for jumps when asset prices are observed with noise-a "swap variance" approach. Journal of Econometrics 144 (2), 352-370.

Jiang, G., Tian, Y., 2005. The model-free implied volatility and its information content. Review of Financial Studies 18 (4), 1305-1342.

Lee, S., 2012. Jumps and information flow in financial markets. Review of Financial Studies 25 (2), $439-479$.

Lee, S., Mykland, P., 2008. Jumps in financial markets: A new nonparametric test and jump dynamics. Review of Financial studies 21 (6), 2535.

Longin, F., Solnik, B., 2001. Extreme correlation of international equity markets. Journal of Finance $56(2), 649-676$.

Mancini, C., 2009. Non-parametric threshold estimation for models with stochastic diffusion coefficient and jumps. Scandinavian Journal of Statistics 36 (2), 270-296.

Mancini, C., Gobbi, F., 2012. Identifying the brownian covariation from the co-jumps given discrete observations. Econometric Theory 28 (2), 249.

Mancino, M., Sanfelici, S., 2011. Estimating covariance via Fourier method in the presence of asynchronous trading and microstructure noise. Journal of Financial Econometrics 9 (2), 367-408.

Podolskij, M., Vetter, M., 2009. Bipower-type estimation in a noisy diffusion setting. Stochastic Processes and their Applications 119, 2803-2831.

Podolskij, M., Vetter, M., 2010. Understanding limit theorems for semimartingales: a short survey. Statistica Neerlandica 64 (3), 329-351.

Podolskij, M., Ziggel, D., 2007. Bootstrapping bipower variation, tech. rep., Ruhr-Universitat Bochum.

Podolskij, M., Ziggel, D., 2010. New tests for jumps in semimartingale models. Statistical inference for 
stochastic processes 13 (1), 15-41.

Todorov, V., 2010. Variance risk-premium dynamics: The role of jumps. Review of Financial Studies 23 (1), 345-383.

Todorov, V., Tauchen, G., 2011. Volatility jumps. Journal of Business and Economic Statistics 29, 356371. 


\section{Appendix A. Theorems and proofs}

Assumption 1. A kernel is a function $K(\cdot): \mathbb{R} \rightarrow \mathbb{R}$, which is differentiable with bounded first derivative almost everywhere in $\mathbb{R}$, and such that $K(0)=1,0 \leq K(\cdot) \leq 1$, and $\lim _{x \rightarrow \infty} K(|x|)=0$. The bandwidth process is a sequence $H_{t, n}$ of processes in $\mathbb{R}^{N}$, which can be written as $H_{t, n}=h_{n} \xi_{t, n}$, where $h_{n}$ is a sequence such that

$$
\begin{aligned}
& \lim _{n \rightarrow \infty} h_{n}=0 \\
& \lim _{n \rightarrow \infty} \frac{1}{h_{n}} \sqrt{\frac{\log n}{n}}=0,
\end{aligned}
$$

and $\xi_{t, n}$ is a vector of $N$ positive adapted stochastic process on $[0, T]$, which are all almost surely (a.s.) bounded with a strictly positive lower bound.

Appendix A.1. The case with finite activity jumps

Assumption 2. $X$ is an $N$-dimensional Ito semi martingale following Eq. (1), where $a_{t}\left(\right.$ in $\mathbb{R}^{N}$ ) and $\boldsymbol{\Sigma}_{t}\left(\right.$ in $\left.\mathbb{R}^{N \times M}\right)$ are càdlàg adapted processes, $W_{t}$ is standard multivariate Brownian motion in $\mathbb{R}^{M}$, and $J_{t}$ is a finite activity jump process of the form $J_{t}^{(i)}=\sum_{k=1}^{N_{t}^{(i)}} \gamma_{\tau_{k}^{(i)}}^{(i)}, i=1, \ldots, N$, where $N_{t}^{(i)}$ is a non explosive counting process and $\gamma_{\tau_{k}^{(i)}}^{(i)}$ are jump sizes at times $\tau_{k}^{(i)}$. Moreover, we assume that the jump sizes are such that $\forall k=1, \ldots$, we have $\mathcal{P}\left(\gamma_{\tau_{k}^{(i)}}^{(i)}=0\right)=0, i=1, \ldots, N$.

Theorem 2. Let the process $X$ satisfy Assumption 2 , and the kernel $K$ and bandwidth $h_{n}$ satisfy Assumption 1. Then, as $n \rightarrow \infty$, we have

$$
\operatorname{SRV}\left(X^{(i)}\right) \stackrel{p}{\longrightarrow} \int_{0}^{T}\left(\sigma^{(i)}\right)_{u}^{2} d u
$$

where $\sigma_{t}^{(i)}$ is the volatility of $X_{t}^{(i)}$.

Proof of Theorem 2. Define threshold realized variance (TRV) as in Mancini (2009):

$$
\operatorname{TRV}^{N}\left(X^{(i)}\right)=\sum_{j=1}^{n}\left(\Delta_{j} X^{(i)}\right)^{2} I_{\left\{\left|\Delta_{j} X^{(i)}\right| \leq H_{j \Delta, n}^{(i)}\right\}}, \quad i=1, \ldots, N
$$

where $I_{A}$ is the indicator function of the set $A$. Now, using the fact that under the assumption $\frac{\Delta_{j} X^{(i)}}{H_{j \Delta . n}}=$ $O_{p}\left(\frac{1}{n^{1 / 2} h_{n}}\right) \rightarrow 0$ [the stochastic bandwidth can be dealt with as in Theorem 2.3 of Corsi et al. 2010, write:

$$
\begin{aligned}
\operatorname{SRV}\left(X^{(i)}\right)-\operatorname{TRV}^{N}\left(X^{(i)}\right)= & \sum_{j=1}^{n}\left(\Delta_{j} X^{(i)}\right)^{2}\left(K\left(\frac{\Delta_{j} X^{(i)}}{H_{j \Delta, n}^{(i)}}\right)-I_{\left\{\left|\Delta_{j} X^{(i)}\right| \leq H_{j \Delta, n}^{(i)}\right\}}\right) \\
= & \sum_{\left|\frac{\Delta_{j} X^{(i)}}{H_{j \Delta, n}}\right| \leq 1}\left(\Delta_{j} X^{(i)}\right)^{2}\left(K\left(\frac{\Delta_{j} X^{(i)}}{H_{j \Delta, n}^{(i)}}\right)-1\right) \\
& +\sum_{\left|\frac{\Delta_{j} X^{(i)}}{H_{j \Delta, n}}\right|>1}\left(\Delta_{j} X^{(i)}\right)^{2} K\left(\frac{\Delta_{j} X^{(i)}}{H_{j \Delta, n}^{(i)}}\right),
\end{aligned}
$$


and

$$
\begin{aligned}
\text { (mean value theorem })= & \sum_{\left|\frac{\Delta_{j} X^{(i)}}{H_{j \Delta, n}}\right| \leq 1}\left(\Delta_{j} X^{(i)}\right)^{2} K^{\prime}\left(\frac{\xi_{j}}{H_{j \Delta, n}^{(i)}}\right) \frac{\Delta_{j} X^{(i)}}{H_{j \Delta, n}^{(i)}}, \\
& +\sum_{j=1}^{n}\left(\Delta_{j} X^{(i)}\right)^{2} K\left(\frac{\Delta_{j} X^{(i)}}{H_{j \Delta, n}^{(i)}}\right) I_{\left\{\left|\Delta_{j} X^{(i)}\right|>H_{j \Delta, n}^{(i)}\right\}},
\end{aligned}
$$

for a suitable random sequence $\xi_{j}$, such that $\left|\xi_{j}\right| \leq\left|\Delta_{j} X^{(i)}\right|$. By the boundedness of $K^{\prime}$ and the bandwidth process and results in Mancini (2009), the absolute value of the first term, i.e. Eq. A.6) is dominated by

$$
C \cdot \operatorname{TRV}^{N}\left(X^{(i)}\right) \frac{\sqrt{\Delta \log \frac{1}{\Delta}}}{h_{n}} \stackrel{p}{\rightarrow} 0,
$$

where $C$ is a suitable constant. For the second term, Eq. A.6], in the case of finite activity Mancini (2009) proves that when $\left|\Delta_{j} X^{(i)}\right|>H_{j \Delta, n}^{(i)}$ only the terms in which jumps occur are left in $\Delta_{j} X^{(i)}$, so that $\frac{\left|\Delta_{j} X^{(i)}\right|}{H_{j \Delta, n}^{(i)}} \stackrel{p}{\rightarrow} \infty$ and by the continuous mapping theorem $K\left(\frac{\Delta_{j} X^{(i)}}{H_{j \Delta, n}^{(i)}}\right) \stackrel{p}{\rightarrow} 0$. Using Corollary 2 in Mancini (2009) we get the desired result [see Mancini and Gobbi, 2012 for a generalizations to the semi martingale case].

Remark 1 introduces a correction to improve the performance of the estimator presented in Theorem 2 in small samples.

Remark 1. (Small sample correction) To improve the finite samples unbiasedness of the estimator defined in Eq. (5), it is advisable to normalize it as

$$
\frac{\sum_{j=1}^{n}\left|\Delta_{j} X^{(i)}\right|^{2} \cdot K\left(\frac{\Delta_{j} X^{(i)}}{H_{j \Delta, n}^{(i)}}\right)}{\Delta \sum_{j=1}^{n} K\left(\frac{\Delta_{j} X^{(i)}}{H_{j \Delta, n}^{(i)}}\right)} \stackrel{p}{\longrightarrow} \int_{0}^{T}\left(\sigma^{(i)}\right)_{u}^{2} d u
$$

as $\Delta \sum_{j=1}^{n} K\left(\frac{\Delta_{j} X^{(i)}}{H_{j \Delta, n}^{(i)}}\right) \stackrel{p}{\longrightarrow} 1$.

Theorem 3. Let the process $X$ satisfy Assumption 2 and the kernel $K$ and bandwidth $h_{n}$ satisfy Assumption 1. Then, as $n \rightarrow \infty$,

$$
\widetilde{\operatorname{SRV}}^{N}\left(X^{(i)}\right) \stackrel{p}{\longrightarrow} \begin{cases}\int_{0}^{T}\left(\sigma^{(i)}\right)_{u}^{2} d u+\sum_{\Delta X_{t}^{(1)} \ldots \Delta X_{t}^{(N)} \neq 0}\left(\Delta X_{t}^{(i)}\right)^{2} & \text { on } \Omega_{T}^{M J, N} \\ \int_{0}^{T}\left(\sigma^{(i)}\right)_{u}^{2} d u & \text { on } \bar{\Omega}_{T}^{N}\end{cases}
$$

where $\sigma_{t}^{(i)}$ is the volatility of $X_{t}^{(i)}$.

Proof of Theorem 3. Write:

$$
\begin{aligned}
\widetilde{\operatorname{SRV}}^{N}\left(X^{(i)}\right)-\operatorname{TRV}^{N}\left(X^{(i)}\right)= & \operatorname{SRV}\left(X^{(i)}\right)-\operatorname{TRV}^{N}\left(X^{(i)}\right) \\
& +\underbrace{\sum_{j=1}^{n}\left(\Delta_{j} X^{(i)}\right)^{2}\left(\prod_{k=1}^{N}\left(1-K\left(\frac{\Delta_{j} X^{(k)}}{H_{j \Delta, n}^{(k)}}\right)\right)\right)}_{A_{n}},
\end{aligned}
$$

where the first term, $\operatorname{SRV}\left(X^{(i)}\right)-\operatorname{TRV}^{N}\left(X^{(i)}\right)$, vanishes in probability asymptotically given Theorem 2 
For the second term, i.e. the summation in $j$, write

$$
\begin{aligned}
A_{n}= & \sum_{\forall k \in 1, \ldots N:\left|\frac{\Delta_{j} X^{(k)}}{H_{j \Delta, n}^{(k)}}\right|>1}\left(\Delta_{j} X^{(i)}\right)^{2}\left(\prod_{k=1}^{N}\left(1-K\left(\frac{\Delta_{j} X^{(k)}}{H_{j \Delta, n}^{(k)}}\right)\right)\right) \\
& +\sum_{\exists k \in 1, \ldots N:\left|\frac{\Delta_{j} X^{(k)}}{H_{j \Delta, n}^{(k)}}\right| \leq 1}\left(\Delta_{j} X^{(i)}\right)^{2}\left(\prod_{k=1}^{N}\left(1-K\left(\frac{\Delta_{j} X^{(k)}}{H_{j \Delta, n}^{(k)}}\right)\right)\right) \\
:=A_{n, 1}+A_{n, 2} &
\end{aligned}
$$

The term $A_{n, 2}$ vanishes in probability because, for the $k$ s such that $\left|\frac{\Delta_{j} X^{(k)}}{H_{j \Delta, n}^{(k)}}\right| \leq 1$, we can write, using the mean value theorem,

$$
1-K\left(\frac{\Delta_{j} X^{(k)}}{H_{j \Delta, n}^{(k)}}\right)=K^{\prime}\left(\xi_{j}^{(k)}\right) \frac{\Delta_{j} X^{(k)}}{H_{j \Delta, n}^{(k)}} \rightarrow 0,
$$

while, for the remaining $k \mathrm{~s}$,

$$
1-K\left(\frac{\Delta_{j} X^{\left(k^{\prime}\right)}}{H_{j \Delta, n}^{\left(k^{\prime}\right)}}\right) \stackrel{p}{\rightarrow} 1
$$

as before. Thus, we remain with $A_{n, 1}$. Consider first the case with the indicator kernel:

$$
\widetilde{A}_{n, 1}:=\sum_{j=1}^{n}\left(\Delta_{j} X^{(i)}\right)^{2} \prod_{k=1}^{N} I_{\left\{\left|\Delta_{j} X^{(k)}\right|>H_{j \Delta, n}^{(k)}\right\}}
$$

which tends to zero on $\bar{\Omega}_{T}^{N}$ Mancini and Gobbi, 2012, straightforward generalization of Theorem 4.2]. Over $\Omega_{T}^{M J, N}, \widetilde{A}_{n, 1}$ tends to $\sum_{\Delta X_{t}^{(1)} \ldots \Delta X_{t}^{(N)} \neq 0}\left(\Delta X_{t}^{(i)}\right)^{2} . \widetilde{A}_{n, 1}$ differs from the realized variance of the process $X^{(i) \prime}$ by a finite number of asymptotically vanishing terms, where $X^{(i) \prime}$ is defined by the sum of continuous part of $X^{(i)}$ and the process of multi-jumps:

$$
X^{(i) \prime}=X^{(i)}-\sum_{t \leq T} \Delta X_{t}^{(i)} I_{\left\{\Delta X_{t}^{(i)} \neq 0 \cap \Delta X_{t}^{(1)} \ldots \Delta X_{t}^{(N)}=0\right\}}
$$

The proof for $A_{n, 1}$ then follows by the continuous mapping theorem as for Theorem 2 .

Theorems 2 and 3 introduce a natural estimator for the multi-jumps on each series. In light of Remark 1. the jump size of stock $i$ corresponding to a multi-jump among all stocks is naturally derived in Remark 2.

\section{Remark 2. (Multi-jump size estimation)}

$$
\frac{\widetilde{\operatorname{SRV}}^{N}\left(X^{(i)}\right)-\operatorname{SRV}\left(X^{(i)}\right)}{\Delta \sum_{j=1}^{n} K\left(\frac{\Delta_{j} X^{(i)}}{H_{j \Delta, n}^{(i)}}\right)} \stackrel{p}{\longrightarrow}\left\{\begin{array}{ll}
\sum_{\Delta X_{t}^{(1)} \ldots \Delta X_{t}^{(N)} \neq 0}\left(\Delta X_{t}^{(i)}\right)^{2} & \text { on } \Omega_{T}^{M J, N} \\
0, & \text { on } \bar{\Omega}_{T}^{N}
\end{array} .\right.
$$

Proof of Theorem 1 Given Theorems 2 and 3 it is sufficient to show that a vector of statistics $S^{\prime}\left(X^{(1)}\right), \ldots, S^{\prime}\left(X^{(N)}\right)$,

$$
S^{\prime}\left(X^{(i)}\right)=\frac{\sum_{j=1}^{n}\left|\Delta_{j} X_{c}^{(i)}\right|^{2}\left(1-\eta_{j}^{i}\right)}{\sqrt{V_{\eta} \mathrm{SQ}\left(X^{(i)}\right)}}
$$


where $X_{c}$ is the purely continuous part of $X$, converges stably in law to multivariate normal distribution with identity covariance matrix. In this case, we can use Theorem 2 in Podolskij and Ziggel (2007) to get the desired result. To this purpose, it is enough to prove that for generic constants $c_{1}, \ldots, c_{N}$ the linear combination

$$
c_{1} S^{\prime}\left(X^{(1)}\right)+\ldots+c_{N} S^{\prime}\left(X^{(N)}\right)
$$

converges to a random normal variable with zero mean and variance $\left(c_{1}^{2}+\ldots+c_{N}^{2}\right)$. To simplify the notation, the proof is carried out in the case $N=2$.

Denote by $\mathbf{E}^{*}[\cdot]$, $\operatorname{Var}^{*}[\cdot]$, and $\mathbf{C o v}^{*}[\cdot]$, respectively, expectation, variance, and covariance conditional on the observed values of $X$. We have

$$
\mathbf{E}^{*}\left[\sum_{j=1}^{n}\left|\Delta_{j} X_{c}^{(i)}\right|^{2}\left(1-\eta_{j}^{i}\right)\right]=\left(1-\mathbf{E}^{*}\left[\eta_{1}^{i}\right]\right) \sum_{j=1}^{n}\left|\Delta_{j} X_{c}^{(i)}\right|^{2}=0,
$$

for any $i$, and

$$
\begin{aligned}
\operatorname{Var}^{*}\left[\sum_{j=1}^{n}\left|\Delta_{j} X_{c}^{(i)}\right|^{2}\left(1-\eta_{j}^{i}\right)\right] & =\left(\mathbf{E}^{*}\left[\left(\eta_{1}^{i}\right)^{2}\right]-\left(\mathbf{E}^{*}\left[\eta_{1}^{i}\right]\right)^{2}\right) \sum_{j=1}^{n}\left|\Delta_{j} X_{c}^{(i)}\right|^{4} \\
& =\operatorname{Var}^{*}\left[\eta_{1}^{i}\right] \sum_{j=1}^{n}\left|\Delta_{j} X_{c}^{(i)}\right|^{4}
\end{aligned}
$$

and

$$
\begin{aligned}
& \operatorname{Cov}^{*}\left[\sum_{j=1}^{n}\left|\Delta_{j} X_{c}^{(i)}\right|^{2}\left(1-\eta_{j}^{i}\right), \sum_{j=1}^{n}\left|\Delta_{j} X_{c}^{(k)}\right|^{2}\left(1-\eta_{j}^{k}\right)\right] \\
& =\mathbf{C o v}^{*}\left[\sum_{j=1}^{n}\left|\Delta_{j} X_{c}^{(i)}\right|^{2} \eta_{j}^{i}, \sum_{j=1}^{n}\left|\Delta_{j} X_{c}^{(k)}\right|^{2} \eta_{j}^{k}\right] \\
& =\left(\mathbf{E}^{*}\left[\eta_{j}^{i} \eta_{j}^{k}\right]-\mathbf{E}^{*}\left[\eta_{j}^{i}\right] \mathbf{E}^{*}\left[\eta_{j}^{k}\right]\right)\left(\sum_{j=1}^{n}\left|\Delta_{j} X_{c}^{(i)}\right|^{2}\right)\left(\sum_{j=1}^{n}\left|\Delta_{j} X_{c}^{(k)}\right|^{2}\right) \\
& =0,
\end{aligned}
$$

where the equivalence to zero of the covariance follows from the independence of $\eta_{j}^{i}$ from $\eta_{j}^{k}$ when $i \neq k$. The rest follows as in Podolskij and Ziggel (2010).

\section{Appendix A.2. The case with infinite activity jumps}

Assumption 3 generalizes the setting of Assumption 2 by allowing for both finite and infinite activity jumps in the multivariate process [see, e.g., Ait-Sahalia and Jacod, 2012 and the references therein for a comprehensive discussion of their role in financial economics]. It has purely theoretical interest, as the jumps of economic interest in this paper are the large ones.

Assumption 3. $X$ is an $N$-dimensional Ito semi martingale following

$$
d X_{t}=a_{t} d t+\boldsymbol{\Sigma}_{t} d W_{t}+d \Xi_{t}
$$

where $a_{t}\left(\right.$ in $\left.\mathbb{R}^{N}\right)$ and $\boldsymbol{\Sigma}_{t}\left(\right.$ in $\left.\mathbb{R}^{N \times M}\right)$ are càdlàg adapted bounded processes, $W_{t}$ is standard multivariate 
Brownian motion in $\mathbb{R}^{M}$, and $\Xi_{t}$ is an infinite activity jump process, with each component being split as

$$
\Xi_{t}^{k}=J_{t}^{(k)}+J_{t}^{(c)}
$$

for $\quad k=1, \ldots, N$. Here, $J_{t}^{(k)}$ is idiosyncratic jump processes, which has no common jumps for different components, and $J_{t}^{(c)}$ is the common jump process. Both $J^{(k)}$ and $J^{(c)}$ are allowed to have infinite activity. For $i \in\{1, \ldots, N, c\}$, the jump processes are defined by

$$
J_{t}^{(k)}=J_{1 t}^{(k)}+J_{2 t}^{(k)}
$$

with

$$
J_{1 t}^{(i)}(\omega)=\int_{0}^{t} \int_{\left|\gamma^{(i)}(\omega, x, s)\right|>1} \gamma^{(i)}(\omega, x, s) \mu^{(i)}(\omega, x, s)
$$

and

$$
J_{2 t}^{(i)}(\omega)=\int_{0}^{t} \int_{\left|\gamma^{(i)}(\omega, x, s)\right| \leq 1} \gamma^{(i)}(\omega, x, s) \tilde{\mu}^{(i)}(\omega, x, s),
$$

where $\mu^{(i)}(\omega, x, s)$ is the Poisson random measure of the jumps, $\tilde{\mu}^{(i)}(\omega, x, s)=\mu^{(i)}(\omega, x, s)$ is its compensated measure, the coefficients $\gamma^{(i)}$ are predictable, and $\int\left(1 \wedge \gamma^{(i)}(\omega, x, s)\right)^{2} d x$ is a.s. finite and locally bounded.

Theorems 4 and Theorem 5 generalize Theores 2 and 3 to the setting of Assumption 3 , with the kernel restricted to be the indicator function. The proof of Theorem 1 remains unchanged.

Theorem 4. Let the process $X$ satisfy Assumption 3, and the kernel $K$ and bandwidth $h_{n}$ satisfy Assumption 1 with $K(x)=I_{\{|x|<1\}}$. Then, as $n \rightarrow \infty$, we have

$$
\operatorname{SRV}\left(X^{(i)}\right) \stackrel{p}{\longrightarrow} \int_{0}^{T}\left(\sigma^{(i)}\right)_{u}^{2} d u
$$

where $\sigma^{(i)}$ is the volatility of $X_{t}^{(i)}$.

Proof of Theorem 4. This is proved in Theorem 4.1 in Mancini (2009).

Theorem 5. Let the process $X$ satisfy Assumption 3 and the kernel $K$ and bandwidth $h_{n}$ satisfy Assumption 1, with $K(x)=I_{\{|x|<1\}}$. Then, as $n \rightarrow \infty$,

$$
\widetilde{\operatorname{SRV}}^{N}\left(X^{(i)}\right) \stackrel{p}{\longrightarrow} \begin{cases}\int_{0}^{T}\left(\sigma^{(i)}\right)_{u}^{2} d u+\left[J^{(c)}\right]_{T} & \text { on } \Omega_{T}^{M J, N} \\ \int_{0}^{T}\left(\sigma^{(i)}\right)_{u}^{2} d u, & \text { on } \bar{\Omega}_{T}^{N}\end{cases}
$$

where $\sigma^{(i)}$ is the volatility of $X_{t}^{(i)}$ and $\left[J^{(c)}\right]_{T}$ is the quadratic variation of the common jump process.

Proof of Theorem 5. Each component of $X$ can be decomposed in a standard way [see, e.g. Podolskij] and Vetter, 2010:

$$
X_{t}^{(k)}=Z_{t}^{(k)}(q)+\tilde{J}_{t}(q), \quad k=1, \ldots, N,
$$

where

$$
\begin{aligned}
Z_{t}^{(k)}(q)=\int_{0}^{t} a_{s}^{(k)} d s+\int_{0}^{t} \sigma_{s} d W_{s} & +\int_{0}^{t} \int_{\left|\gamma^{(k)}(\omega, x, s)\right|>1 / q} \gamma^{(k)}(\omega, x, s) \mu^{(k)}(\omega, x, s) \\
& +\int_{0}^{t} \int_{\left|\gamma^{(c)}(\omega, x, s)\right|>1 / q} \gamma^{(c)}(\omega, x, s) \mu^{(c)}(\omega, x, s)
\end{aligned}
$$


and

$$
\tilde{J}_{t}(q)=\int_{0}^{t} \int_{\left|\gamma^{(k)}(\omega, x, s)\right| \leq 1 / q} \gamma^{(k)}(\omega, x, s) \mu^{(k)}(\omega, x, s)+\int_{0}^{t} \int_{\left|\gamma^{(c)}(\omega, x, s)\right| \leq 1 / q} \gamma^{(c)}(\omega, x, s) \mu^{(c)}(\omega, x, s) .
$$

That is, $Z_{t}^{(k)}(q)$ and $\tilde{J}_{t}(q)$ are, respectively, continuous part plus finite activity large jumps and infinite activity small jumps.

Because the process $Z_{t}^{(k)}(q)$ has jumps of finite activity from the theorem for the finite activity case, we have

$$
\widetilde{\operatorname{SRV}}^{N}\left(Z^{(i)}(q)\right) \stackrel{p}{\longrightarrow}\left\{\begin{array}{ll}
\int_{0}^{T}\left(\sigma^{(i)}\right)_{u}^{2} d u+\left[J^{(c)}(q)\right]_{T} & \text { on } \Omega_{T}^{M J, N} \\
\int_{0}^{T}\left(\sigma^{(i)}\right)_{u}^{2} d u, & \text { on } \bar{\Omega}_{T}^{N}
\end{array} ;\right.
$$

where $\left[J^{(c)}(q)\right]_{T}$ is the quadratic variation the jump process

$$
J_{t}^{c}(q)=\int_{0}^{t} \int_{\left|\gamma^{(c)}(\omega, x, s)\right|>1 / q} \gamma^{(c)}(\omega, x, s) \mu^{(c)}(\omega, x, s) .
$$

As $q \rightarrow \infty$, the quadratic variation of $J_{t}^{c}(q)$ converges to the quadratic variation of $J_{t}^{c}$ [see the proof of the Eq. (27) in Podolskij and Vetter 2010]. Hence, it is sufficient to show

$$
\widetilde{\operatorname{SRV}}^{N}\left(X^{(i)}\right)-\widetilde{\operatorname{SRV}}^{N}\left(Z^{(i)}(q)\right) \rightarrow^{p} 0 .
$$

Consider the decomposition:

$$
\begin{aligned}
\widetilde{\operatorname{SRV}}^{N}\left(X^{(i)}\right)-\widetilde{\operatorname{SRV}}^{N}\left(Z^{(i)}(q)\right) & =\sum_{j=1}^{n}\left|\Delta_{j} Z^{(i)}(q)\right|^{2}\left(I_{\left\{\left|\Delta_{j} X^{(i)}\right| \leq H_{j \Delta, n}^{(i)}\right\}}-I_{\left\{\left|\Delta_{j} Z^{(i)}(q)\right| \leq H_{j \Delta, n}^{(i)}\right\}}\right) \\
& +\sum_{j=1}^{n}\left|\Delta_{j} Z^{(i)}(q)\right|^{2}\left(\prod_{k=1}^{N}\left(1-I_{\left\{\left|\Delta_{j} X^{(k)}\right| \leq H_{j \Delta, n}^{(k)}\right\}}\right)-\prod_{k=1}^{N}\left(1-I_{\left\{\left|\Delta_{j} Z^{(k)}(q)\right| \leq H_{j \Delta, n}^{(k)}\right\}}\right)\right) \\
& +2 \sum_{j=1}^{n}\left|\Delta_{j} Z^{(i)}(q)\right|\left|\Delta_{j} \tilde{J}(q)^{(i)}\right|\left(I_{\left\{\left|\Delta_{j} X^{(i)}\right| \leq H_{j \Delta, n}^{(i)}\right\}}+\prod_{k=1}^{N}\left(1-I_{\left\{\left|\Delta_{j} X^{(i)}\right| \leq H_{j \Delta, n}^{(i)}\right\}}\right)\right) \\
& +\sum_{j=1}^{n}\left|\Delta_{j} \tilde{J}(q)^{(i)}\right|^{2}\left(I_{\left\{\left|\Delta_{j} X^{(i)}\right| \leq H_{j \Delta, n}^{(i)}\right\}}+\prod_{k=1}^{N}\left(1-I_{\left\{\left|\Delta_{j} X^{(i)}\right| \leq H_{j \Delta, n}^{(i)}\right\}}\right)\right) .
\end{aligned}
$$

The first summation in A.35 can be written as

$$
\begin{aligned}
& \underbrace{\sum_{j=1}^{n}\left|\Delta_{j} Z^{(i)}(q)\right|^{2}\left(I_{\left\{\left|\Delta_{j} X^{(i)}\right| \leq H_{j \Delta, n}^{(i)}\right\}}-I_{\left\{\left|\Delta_{j} Z^{(i)}(q)\right| \leq 2 H_{j \Delta, n}^{(i)}\right\}}\right)}_{A_{1}} \\
& +\underbrace{\sum_{j=1}^{n}\left|\Delta_{j} Z^{(i)}(q)\right|^{2}\left(I_{\left\{\left|\Delta_{j} Z^{(i)}(q)\right| \leq 2 H_{j \Delta, n}^{(i)}\right\}}-I_{\left\{\left|\Delta_{j} Z^{(i)}(q)\right| \leq H_{j \Delta, n}^{(i)}\right\}}\right)}_{A_{2}},
\end{aligned}
$$

where the term $A_{2}$ converges in probability to zero because both the terms in the difference converge to the same probability limit $\int_{0}^{T}\left(\sigma^{(i)}\right)_{u}^{2} d u$. The term $A_{1}$ can be dealt as the term in Eq. (21) in Mancini (2009) to show that it converges to zero in probability. 
Denoting by $\mathcal{P}(A)$ the power set of the set $A$ and by $|B|$ is the cardinality of the set $B$, we have

$$
\prod_{k=1}^{N}\left(1-I_{\left\{\left|\Delta_{j} X^{(k)}\right| \leq H_{j \Delta, n}^{(k)}\right\}}\right)=\sum_{B \in \mathcal{P}(\{1, \ldots, N\})}(-1)^{|B|} \prod_{s \in B} I_{\left\{\left|\Delta_{j} X^{(s)}\right| \leq H_{j \Delta, n}^{(s)}\right\}},
$$

and we can write the second summation in the decomposition A.35 as

$$
\sum_{j=1}^{n}\left|\Delta_{j} Z^{(i)}(q)\right|^{2} \sum_{B \in \mathcal{P}(\{1, \ldots N\})}(-1)^{|B|}(\underbrace{\prod_{s \in B} I_{\left\{\left|\Delta_{j} X^{(s)}\right| \leq H_{j \Delta, n}^{(s)}\right\}}-\prod_{s \in B} I_{\left\{\left|\Delta_{j} Z^{(s)}(q)\right| \leq H_{j \Delta, n}^{(s)}\right\}}}_{\psi_{B}}) .
$$

We have

$$
\psi_{B}=I_{\left\{\forall s \in B:\left|\Delta_{j} X^{(s)}\right| \leq H_{j \Delta, n}^{(s)}, \exists \bar{s} \in B:\left|\Delta_{j} Z^{(\bar{s})}(q)\right|>H_{j \Delta, n}^{(\bar{s})}\right\}}-I_{\left\{\forall s \in B:\left|\Delta_{j} Z^{(s)}(q)\right| \leq H_{j \Delta, n}^{(s)}, \exists \bar{s} \in B:\left|\Delta_{j} X^{(\bar{s})}\right|>H_{j \Delta, n}^{(\bar{s})}\right\}} .
$$

Now, consider the first indicator function. Clearly,

$$
I_{\left\{\forall s \in B:\left|\Delta_{j} X^{(s)}\right| \leq H_{j \Delta, n}^{(s)}, \exists \bar{s} \in B:\left|\Delta_{j} Z^{(\bar{s})}(q)\right|>H_{j \Delta, n}^{(\bar{s})}\right\}} \leq \sum_{s \in B} I_{\left\{\left|\Delta_{j} Z^{(s)}(q)\right|>H_{j \Delta, n}^{(s)}\right\}}
$$

For each indicator function in the sum, we have

$$
\left.\mathbf{E}\left[I_{\left\{\left|\Delta_{j} Z^{(s)}(q)\right|>H_{j \Delta, n}^{(s)}\right\}}\right]=\mathcal{P}\left(\left|\Delta_{j} Z^{(s)}(q)\right|>H_{j \Delta, n}^{(s)}\right\}\right) \leq \frac{1}{C} \frac{\mathbf{E}\left[\left|\Delta_{j} Z^{(s)}(q)\right|\right]}{h_{n}} \leq C^{\prime} \frac{\Delta^{1 / 2}}{h_{n}},
$$

where we use the Markov inequality, the lower bound $H_{j \Delta, n}^{(s)} \geq C h_{n}$, and the constant $C^{\prime}$ is the same for all $j$ given the boundedness of the driving coefficients. Because the sum over $s \in B$ contains at most $N$ terms, this proves that $\phi_{B}=O_{p}\left(\Delta^{1 / 2} / h_{n}\right)$ (as the second indicator function also can be treated in the same way). Also because $\mathcal{P}(\{1, \ldots, N\})$ contains a finite number of terms, it follows that the term in Eq. A.38 is negligible in probability.

The last two terms in Eq. A.35 are negligible in probability by similar reasons. The quantities

$$
\sum_{j=1}^{n}\left|\Delta_{j} Y^{(i)}\right|\left|\Delta_{j} J_{2}^{(i)}\right|\left(I_{\left\{\left|\Delta_{j} X^{(i)}\right| \leq H_{j \Delta, n}^{(i)}\right\}}\right)
$$

and

$$
\sum_{j=1}^{n}\left|\Delta_{j} J_{2}^{(i)}\right|^{2}\left(I_{\left\{\left|\Delta_{j} X^{(i)}\right| \leq H_{j \Delta, n}^{(i)}\right\}}\right)
$$

are negligible as, respectively, the third and the fourth summations in the decomposition (20) in the proof of Theorem 4 in Mancini (2009). Now, from the fact that, for every integer $N$, the probabilities of the events

$$
\left\{\sum_{j=1}^{n}\left|\Delta_{j} Y^{(i)}\right|\left|\Delta_{j} J_{2}^{(i)}\right| \prod_{k=1}^{N} I_{\left\{\left|\Delta_{j} X^{(i)}\right| \leq H_{j \Delta, n}^{(i)}\right\}}>\epsilon\right\}
$$

and

$$
\left\{\sum_{j=1}^{n}\left|\Delta_{j} J_{2}^{(i)}\right|^{2} \prod_{k=1}^{N} I_{\left\{\left|\Delta_{j} X^{(i)}\right| \leq H_{j \Delta, n}^{(i)}\right\}}>\epsilon\right\}
$$


are not larger than the probabilities of

$$
\left\{\sum_{j=1}^{n}\left|\Delta_{j} Y^{(i)}\right|\left|\Delta_{j} J_{2}^{(i)}\right| I_{\left\{\left|\Delta_{j} X^{(i)}\right| \leq H_{j \Delta, n}^{(i)}\right\}}>\epsilon\right\}
$$

and

$$
\left\{\sum_{j=1}^{n}\left|\Delta_{j} J_{2}^{(i)}\right|^{2} I_{\left\{\left|\Delta_{j} X^{(i)}\right| \leq H_{j \Delta, n}^{(i)}\right\}}>\epsilon\right\},
$$

respectively, the desired result follows.

\section{Appendix B. Implementation details}

The multi-jump test used in this paper is computed on five minute returns obtained aggregating one minute returns. The final test is the average of the five tests computed on the possible grids. The same procedure is applied to univariate jump tests, that is, they are computed as the average of the tests over the five possible grids. The exact position of the multi-jumps is located taking the grid with the maximum value of the test and selecting the five minute interval with the largest product $\prod_{k=1}^{N}\left(1-K\left(\frac{\Delta_{j} X^{(k)}}{H_{j \Delta, n}^{(k)}}\right)\right)$.

\section{Appendix B.1. Smoother and bandwidth selection}

Numerical experiments show that the test is more stable when the kernel is continuous and that the kernel shape is not crucial. In simulations and empirical work, we use a (normalized) Gaussian function

$$
K(x)=e^{-x^{2} / 2} .
$$

For the choice of the bandwidth process in Eq. (6), we first estimate instantaneous volatility in two steps. In the first step, we compute the estimate as in Corsi et al. (2010). In the second step, we apply a correction factor based on the intraday position of the return. The correction factor is the ratio of the average (across days) intraday squared return and the average (across days) intraday variance obtained with the first step. This second step is meant to recover, on average, the same intraday pattern of squared returns. As typically found in the literature, the correction factor is higher for returns close to the opening and the closing times of the market.

The bandwidth parameter $h_{n}$ is selected by an automated procedure based on simulations. Because we normalize to local variance, we replicate the null hypothesis by simulating $N$ i.i.d. standard Gaussian variates correlated as in the data (we use the average correlation matrix in the sample) and then add a given number $M$ of multi-jumps (with $M<N$ ) of desired size. For each set of replications, we then find $h_{n}$ such that the average size of the test, computed on replications, is $5 \%$ at $95 \%$ confidence intervals. Such a bandwidth should be used for testing, approximately, for at least $M+1$ multi-jumps. Figure B.14 shows the result for different $N$ and $M$. Matlab ${ }^{\circledR}$ code for automated bandwidth selection, and for the multi-jump test, is available upon request. 


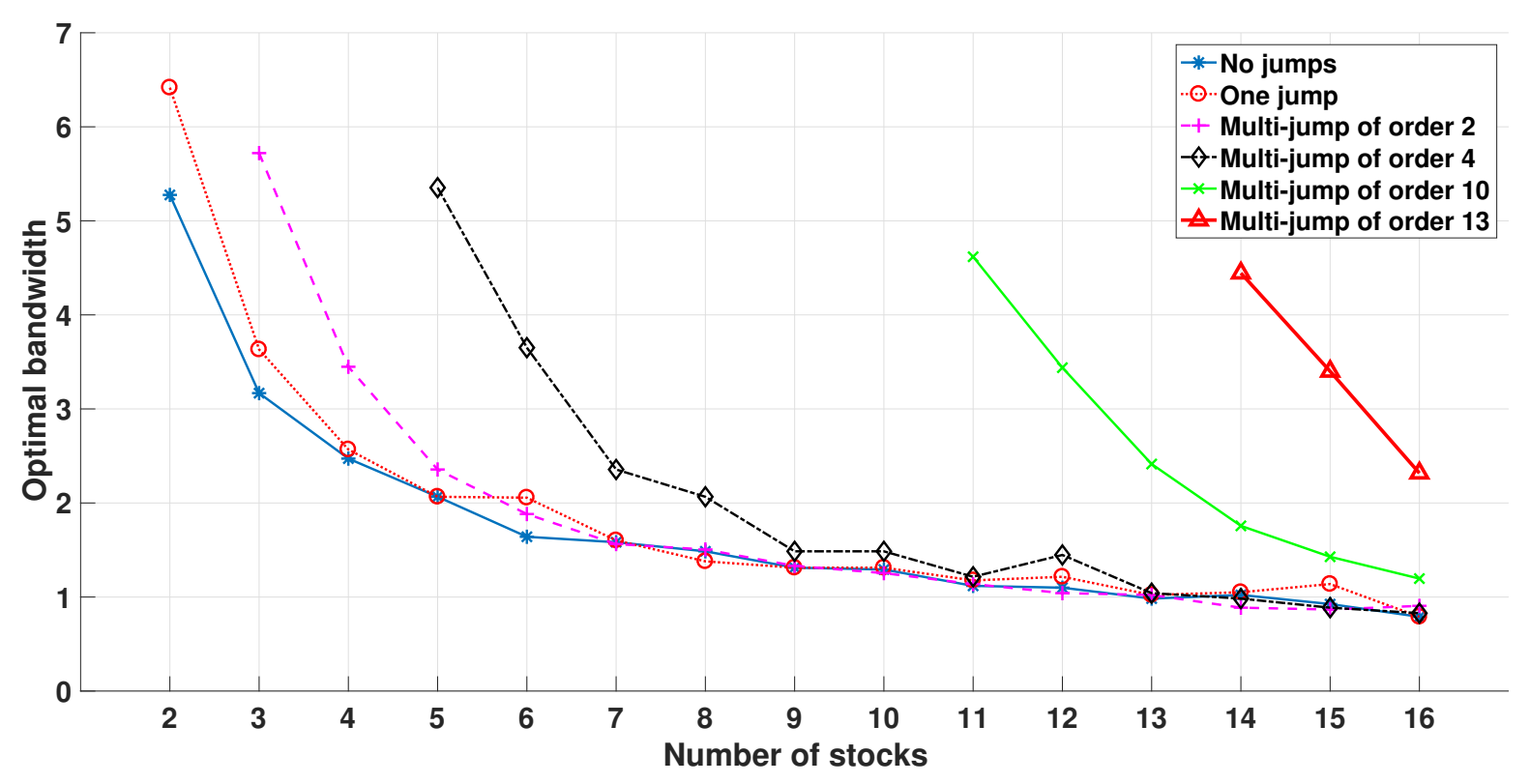

Figure B.14: Bandwidth selection rule. The figure shows the optimal bandwidth parameter $h_{n}$ obtained by calibrating the correct size on simulated experiments in which the null hypothesis is without jumps or contaminated by one jump, or multi-jumps of order $2,4,10$ and 13 , for various values of the number of stocks. For example, the choice with $h_{n} \simeq 6.5(4.5)$ is the most conservative with $N=2(N=16)$, and $h_{n} \simeq 1$ is the less conservative.

The number of multi-jumps under the null affects the bandwidth, but only slightly. The effect is stronger when the number of multi-jumps under the null approaches $N$. A conservative choice should then be $h_{n}=6.5$ (for $N=2$ ) or $h_{n}=4.5$ (for $N=16$ ). For all $N$, the choice $h_{n} \simeq 1$ is the less conservative. For $N=16$, the range should then be $1 \leq h_{n} \leq 3$, depending on which kind of signal one is interested in. This is beneficial to the testing procedure because when a multi-jump occurs, some of the associated jumps could be small or could happen with a small lag.

The bandwidth used in the empirical application is obtained as follows. We compute, for every value of $N$, the automated bandwidth $\widetilde{h}(N)$ using the procedure described above at a given confidence interval as a function of $N$, under the null of zero jumps. We then calibrate $\widetilde{h}(N)$ with the function $h(N)=c_{\alpha}+c / N$, where $c_{\alpha}$ depends on the confidence interval and $c$ is a global constant, which is fit to the value $\widehat{c}=9.57$ reported in Section 4

\section{Appendix B.2. Univariate jump tests}

Here we discuss the implementation of the univariate jump tests and the Jacod and Todorov multijump test used in the Monte Carlo experiments and in the empirical applications.

We start with univariate tests, for which we use three tests: BNS, CPR and ABD. The null hypothesis for all three tests is the absence of jumps. The BNS test introduced by Barndorff-Nielsen and Shephard (2006) is based on the comparison of the realized variance and bipower variation, which are, respectively, non-robust and robust to jumps measures of the integrated variance. For a one-dimensional process $X$, the BNS test statistic has the form

$$
\Delta^{-1 / 2} \frac{1-\frac{\operatorname{MV}(X ;[1,1])}{\operatorname{RV}(X)}}{\sqrt{(\pi / 4+\pi-5) \max \left(1, \frac{\operatorname{MV}(X ;[4 / 3,4 / 3,4 / 3])}{\operatorname{MV}(X ;[1,1])}\right)}} \Rightarrow \mathcal{N}(0,1),
$$


where the convergence in distribution holds under the null

$$
\operatorname{MV}\left(X ;\left[r_{1}, r_{2}, \ldots, r_{m}\right]\right)=\left(\prod_{j=1}^{m}\left(\mu_{r_{j}}\right)^{-1}\right)(\Delta)^{1-\frac{r_{1}+\ldots+r_{m}}{2}} \cdot \sum_{i=1}^{n-m+1} \prod_{j=1}^{m}\left|\Delta_{i+j} X\right|^{r_{j}}
$$

and

$$
\mathrm{RV}(X)=\operatorname{MV}(X ;[2,0, \ldots, 0]) .
$$

The CPR test, introduced by Corsi et al. (2010), is similar to BNS. However, it uses threshold bipower variation instead of simple bipower variation. Moreover, the special finite sample correction is applied to the test statistic to improve the size of the test in finite samples. The test statistic has the following form

$$
\Delta^{-1 / 2} \frac{(\operatorname{RV}(X)-\mathrm{C}-\operatorname{TMV}(X ;[1,1])) \operatorname{RV}(X)^{-1}}{\sqrt{(\pi / 4+\pi-5) \max \left(1, \frac{\mathrm{C}-\operatorname{TMV}(X ;[4 / 3,4 / 3,4 / 3])}{\mathrm{C}-\operatorname{TMV}^{2}(X ;[1,1])}\right)}} \Rightarrow \mathcal{N}(0,1),
$$

where the convergence in distribution holds under the null and C-TMV $(X ; \mathbf{r})$ is the corrected version of threshold multipower estimator. The correction consists of replacing returns $\Delta_{j} X>H_{j}$ by their expectations under the assumption $\Delta_{j} X \sim \mathcal{N}\left(0, \sigma_{j}^{2}\right)$, leading to

$$
\operatorname{C-TMV}(\mathbf{r})=\left(\prod_{j=1}^{m}\left(\mu_{r_{j}}\right)^{-1}\right)(\Delta)^{1-\frac{r_{1}+\ldots+r_{m}}{2}} \cdot \sum_{i=1}^{n-m+1} \prod_{j=0}^{m-1} Z_{r}\left(\Delta X_{i+j}, H_{i+j}\right),
$$

where the function $Z_{r}(x, y)$ is

$$
Z_{r}(x, y)=\left\{\begin{array}{ll}
|x|^{r} & \text { if } x^{2} \leq y \\
\frac{1}{2 N\left(-c_{\vartheta}\right) \sqrt{\pi}}\left(\frac{2}{c_{\vartheta}^{2}} y\right)^{\frac{\gamma}{2}} \Gamma\left(\frac{r+1}{2}, \frac{c_{\vartheta}^{2}}{2}\right) & \text { if } x^{2}>y
\end{array} .\right.
$$

To implement the CPR test, we always use the threshold threshold defined according to Eq. (6) with the constant $h_{n}=3$.

The ABD test is a modification of the Lee and Mykland (2008) test, which considers the set of all intraday standardized returns

$$
z_{i}=\Delta_{i} X / \sqrt{V_{i}}
$$

where $V_{i}$ is the estimate of spot volatility at time corresponding to the intraday return number $i, i=$ $1, \ldots, n$. Under the null, each $z_{i}$ is asymptotically standard normal. Hence, one can test the absence of jumps by comparing all standardized intraday returns with the normal critical values. To guarantee that the daily first type error does not exceed a given level $\alpha$, the size of each intraday test must be equal to $\beta=1-(1-\alpha)^{\Delta}$.

Table B.4 shows the size and power of the considered tests, as computed on the simulated stocks also used in Section 7.1. We confirm the findings in the literature [see Dumitru and Urga, 2012 for a wider comparison]. The most powerful test is ABD, but at the cost of a distorted size. CPR and BNS are correctly sized, but CPR has higher power, thus striking a superior balance. For this reason, we mainly use CPR for detecting univariate jumps in the empirical application in Section 4. 
Table B.4: Size and power of univariate jump tests. This table shows the size and power of univariate jump tests tests on simulated data. The sampling frequency $n=80$ corresponds to five-minute intraday observations. CPR $=$ Corsi, Pirino and Renò; BNS=Barndorff-Nielsen and Shephard; ABD=Andersen, Bollerslev and Dobrev.

\begin{tabular}{|c|c|c|c|c|}
\hline & \multicolumn{4}{|c|}{ Confidence interval } \\
\hline & $90 \%$ & $95 \%$ & $99 \%$ & $99.9 \%$ \\
\hline \multicolumn{5}{|c|}{ Case 1: Standard settings } \\
\hline \multicolumn{5}{|c|}{ Univariate tests on individual stocks: power } \\
\hline CPR & 91.2 & 89.0 & 84.4 & 73.9 \\
\hline BNS & 86.0 & 81.9 & 71.5 & 56.1 \\
\hline $\mathrm{ABD}$ & 97.0 & 96.6 & 95.1 & 92.8 \\
\hline \multicolumn{5}{|c|}{ Univariate tests on individual stocks: size } \\
\hline CPR & 9.2 & 5.3 & 2.1 & 0.0 \\
\hline BNS & 8.6 & 4.8 & 1.9 & 0.0 \\
\hline $\mathrm{ABD}$ & 17.3 & 10.8 & 3.0 & 0.8 \\
\hline \multicolumn{5}{|c|}{ Case 2: High volatility of volatility } \\
\hline \multicolumn{5}{|c|}{ Univariate tests on individual stocks: power } \\
\hline CPR & 71.7 & 67.7 & 61.2 & 54.8 \\
\hline BNS & 64.9 & 61.5 & 55.2 & 49.5 \\
\hline $\mathrm{ABD}$ & 96.5 & 95.9 & 93.7 & 90.0 \\
\hline \multicolumn{5}{|c|}{ Univariate tests on individual stocks: size } \\
\hline CPR & 28.5 & 20.9 & 12.1 & 6.0 \\
\hline BNS & 18.1 & 11.3 & 5.5 & 2.0 \\
\hline $\mathrm{ABD}$ & 95.6 & 94.3 & 91.3 & 86.9 \\
\hline
\end{tabular}

Appendix B.3. Quadratic variation measures

To measure the continuous covariations among asset prices, we use an approach based on the polarization of bipower variation, as in Barndorff-Nielsen and Shephard (2003), adapted for threshold bipower variations (Corsi et al. 2010). Let $\operatorname{Cov}_{t}^{(i, j)}$ denote a measure of continuous quadratic covariation of two stocks $i$ and $j$ at day $t$, and let $V_{t}^{(i)}=\operatorname{Cov}_{t}^{(i, i)}$ be the measure of continuous integrated variance of stock $i$ at day $t$. We first denote, for $j=1, \ldots, n$ by

$$
\triangleleft_{j} X=\left(\Delta_{j} X\right) I_{\left\{\left|\Delta_{j} X\right| \leq \theta_{j}\right\}},
$$

where $\theta_{j}$ is a threshold computed as in Corsi et al. (2010), $\psi_{j} X$ are the truncated returns and we denote by $X$ the corresponding cumulated. We then set

$$
\operatorname{Cov}_{t}^{(i, j)}=\frac{1}{4}\left(\mathrm{BV}\left(X^{(i)}+X^{(j)}\right)-\mathrm{BV}\left(\left.X\right|^{(i)}-X^{(j)}\right)\right)
$$

where

$$
\mathrm{BV}(X)=\left(\mu_{1}\right)^{-2} \sum_{j=1}^{\lfloor T / \Delta\rfloor-1}\left|\triangleleft_{j} X\right|\left|\triangleleft_{j+1} X\right|
$$

is threshold bipower variation, with $\mu_{1}=\sqrt{2 / \pi}$. $C o v_{t}^{(i, j)}$ is a consistent and jump-robust estimator of the continuous part of the covariation process of the log-price processes $X^{(i)}$ and $X^{(j)}$. 
The intraday measure of correlation between two stocks is defined by

$$
\operatorname{Corr}_{t}^{(i, j)}=\frac{\operatorname{Cov}_{t}^{(i, j)}}{\sqrt{V_{t}^{(i)} V_{t}^{(j)}}} .
$$

It can be mapped into the whole real line by the use of Fisher transformation

$$
\widetilde{\rho}_{t}^{(i, j)}=\frac{1}{2} \log \frac{1+\operatorname{Corr}_{t}^{(i, j)}}{1-\operatorname{Corr}_{t}^{(i, j)}},
$$

which is used in regression analysis in Subsection 6.3.

\section{Appendix C. SURE representation and estimation}

This Appendix describes the estimation and testing approach adopted in the empirical analyses to verify the joint significance of multi-jumps coefficients. Given that our purpose is to test restrictions across equations, a simultaneous equation system must be considered. The most appropriate setting is that of seemingly unrelated regression equations (SURE) which reads as

$$
\left[\begin{array}{l}
Y_{1} \\
Y_{2} \\
\vdots \\
Y_{m}
\end{array}\right]=\left[\begin{array}{cccc}
X_{1} & 0 & \ldots & 0 \\
0 & X_{2} & \ldots & 0 \\
\vdots & & \ddots & \vdots \\
0 & 0 & \ldots & X_{m}
\end{array}\right]\left[\begin{array}{c}
\beta_{1} \\
\beta_{2} \\
\vdots \\
\beta_{m}
\end{array}\right]+\left[\begin{array}{c}
\epsilon_{1} \\
\epsilon_{2} \\
\vdots \\
\epsilon_{m}
\end{array}\right]
$$

where $Y_{j}$ is a $T$-dimensional vector containing the sample data for the jth dependent variable, $X_{j}$ is the $T \times K_{j}$ matrix of explanatory variables in the jth equation, and $\beta_{j}$ is the associated $K_{j}$-dimensional vector of regression coefficients. In addition, the error terms are assumed to be homoskedastic, serially uncorrelated, and not cross-correlated, but are contemporaneously correlated, implying that $\mathbb{E}\left[\epsilon \epsilon^{\prime}\right]=$ $\Omega \otimes I_{T}, \Omega$ being the covariance matrix such that $\mathbb{E}\left[\epsilon_{t} \epsilon_{t}^{\prime}\right]$ with $\epsilon_{t}=\left[\epsilon_{1, t}, \epsilon_{2, t}, \ldots, \epsilon_{m, t}\right]$. In our case, the dependent variable $Y_{j}$ contains either the sample data of the log-realized volatility for one stock, $Y_{j}=\log V_{t}^{(i)}$, or the sample data of the Fisher transformation of realized correlations between two stocks, $Y_{j}=\tilde{\rho}_{t}^{(i, l)}$. In the first case, $m=16$; in the second case $m=120$. Moreover, $X_{j}$ could contain HAR terms as well as jump and multi-jump indicator variables. The matrices $X_{j}, j=1,2, \ldots m$ are specific to each dependent variable and, thus, to each equation, but include both equation-specific elements, the HAR terms, jump and multi-jump variables, and common elements, when the multi-jump indicator variable is taken into account. The SURE model allows testing cross-equation restrictions when the errors are contemporaneously correlated. In the present study, we cannot exclude a priori that the innovations of different equations are independent.

In a SURE model, generalized least squares (GLS) estimation is required to take into account the covariance structure of the innovations. Feasible GLS estimation is normally performed, by first applying ordinary least squares (OLS) on a univariate basis, then recovering the covariance of innovations from the univariate regression residuals and, finally, applying GLS with the estimated residuals covariance.

Nevertheless, the use of FGLS requires a diagnostic check on the first stage residuals, given the presence of lagged dependent variables (the HAR terms) on the explanatory variables $X_{j}$, and because the true unrestricted model is of a vector auto regression (VAR) type [the general model is a VARX $(22)$ 
whose parameters are highly restricted in the VAR part]. In fact, if the first stage residuals would be serially correlated, this could lead to biases in the first stage OLS. Those biases might be associated with a correlation between regressors and innovations. Differently, if first stage residuals show evidence of serial cross-correlations, those, on the one hand, would signal the need of a less restricted HAR component, for instance, allowing for interactions across equations, and, on the other hand, would lead to biases due to the omitted variable problem.

In our empirical analyses, diagnostic checks on the first stage residuals show that those problems are not a real concern. In very few cases, diagnostic tests lead to a rejection of the null hypotheses, and the first lags in the autocorrelation and cross-correlation functions were showing values at maximum equal to 0.1. Therefore, we believe those results support the use of FGLS. However, diagnostic tests show evidence of heteroskedasticity. We take them into account in the estimation of Feasible GLS standard errors by adopting a White-type correction. We replace the traditional FGLS estimator of the parameter covariance matrix with the expression

$$
\mathbb{V}\left[\beta_{F G L S}\right]=\left(X^{\prime}\left(\hat{\Omega}^{-1} \otimes I_{T}\right) X\right)^{-1} S\left(X^{\prime}\left(\hat{\Omega}^{-1} \otimes I_{T}\right) X\right)^{-1}
$$

where

$$
S=\sum_{t=1}^{T} X_{t} \hat{\Omega}^{-1} \varepsilon_{t} \varepsilon_{t}^{\prime} \hat{\Omega}^{-1} X_{t}^{\prime}
$$

and $\Omega$ is estimated on the first stage regression residuals. The matrix $X_{t}$ is block-diagonal with diagonal elements equal to the column-vector $X_{j, t}$. It contains the time $t$ explanatory variables for equation $j$. 